\title{
CRITICAL FIELD CURVES FOR THE ThU ALLOY SYSTEM
}

\author{
Ph.D. Thes is Submitted to lowa State University, \\ February, 1973
}

H. L. Watson

\author{
Ames Laboratory, USAEC \\ lowa State University \\ Ames, lowa 50010
}

\begin{abstract}
This report was prepared as an account of work This the United States nor the United States Atomic Energy the United States nor the Unir employees, nor any of Commission, nor any of their employes, their contractors, subcontractors, or their employes, makes any warranty, express or implied, or assumes any

legal liability or responsinility for the arrinary, nm

pleteness or usefulness of any information, apparatus,

product or process disclosed, or represents that its use would not infringe privately owned rights.
\end{abstract}

Date of Manuscript: February, 1973

PREPARED FOR THE U. S. ATOMIC ENERGY COMMISSION DIVISION OF RESEARCH UNDER CONTRACT NO. W-7405-eng-82 


\section{DISCLAIMER}

This report was prepared as an account of work sponsored by an agency of the United States Government. Neither the United States Government nor any agency Thereof, nor any of their employees, makes any warranty, express or implied, or assumes any legal liability or responsibility for the accuracy, completeness, or usefulness of any information, apparatus, product, or process disclosed, or represents that its use would not infringe privately owned rights. Reference herein to any specific commercial product, process, or service by trade name, trademark, manufacturer, or otherwise does not necessarily constitute or imply its endorsement, recommendation, or favoring by the United States Government or any agency thereof. The views and opinions of authors expressed herein do not necessarily state or reflect those of the United States Government or any agency thereof. 


\section{DISCLAIMER}

Portions of this document may be illegible in electronic image products. Images are produced from the best available original document. 
This report was prepared as an account of work sponsored by the United States Government. Neither the United States nor the United States Atomic Energy Commission, nor any of their employees, nor any of their contractors, subcontractors, or their employees, makes any warranty, express or implied, or assumes any legal liability or responsibility for the accuracy, completeness or usefulness of any information, apparatus, product or process disclosed, or represents that its use would not infringe privately owned rights.

Available from: National Technical Information Service Department $A$

Springfield, VA 22151

Price: Microfiche $\$ 0.95$ 
Critical field curves for the ThU alloy system

by

Harlan Leroy Watson

A Dissertation Submitted to the

Graduate Faculty in partial Fulfillment of

The Requirements for the Degree of

DOCTOR OF PHILOSOPHY

Department: Physics

Major: Solid state Physics

Approved:

D. K. Finnemore

In Charge of Major Work

For the Major Department

For the Graduate College

Iowa state University

Ames, Iowa

1973 
TABLE OF CONTENTS

\section{ABSTRACT}

I. INTRODUCTION

A. Historical Survey 1

B. Thermodynamic Theory of the superconducting Transition

C. BCS Microscopic Theory of Superconductivity 9

D. Addition of Impurities to a Pure Metal 13

E. Previous Measurements on ThU 42

F. Objectives 45

II. EXPERIMENTAL APPARATUS AND PROCEDURE . 46

A. Sample Preparation and Analysis 46

B. Cryostat 47

C. Thermometry and Temperature Control 50

D. Magnetic Field Sources 53

E. Mutual Inductance Bridge . 57

F. Critical Field Measurements 60

III. RESULTS AND DISCUSSION $\quad 62$

$\begin{array}{ll}\text { IV. SUMMARY } & 122\end{array}$

$\begin{array}{ll}\text { V. BIBLIOGRAPHY } & 124\end{array}$

VI. ACKNOWLEDGMENTS 132

VII. APPENDIX A: CRITICAL FIELD DATA 133

VIII. APPENDIX B: $H_{0}$ AND $D(t)$ FROM BCS THEORY 140

IX. APPENDIX $c: \mathrm{H}_{\mathrm{O}}$ AND $\mathrm{D}(t)$ FROM THE ANISOTROPY CORRECTED BCS THEORY OF CLEM 151 
Critical field curves for the Thu alloy system

Harlan Leroy Watson

Under the supervision of D. K. Finnemore

From the Department of Physics

Iowa State University

The superconducting critical field curves have been measured for the ThU alloy system and have been found to be in agreement with the BCS theory critical field curves to an accuracy of $1 \%$, thus providing additional experimental evidence that the rapid depression of the superconducting transition temperature of pure thorium by the uranium impurities is due to pair-weakening rather than to pair-breaking mechanisms. Additional experimental evidence is provided by the fact that the reduced specific heat jumps at $\mathrm{T}_{\mathrm{c}}$ for these alloys, obtained from the slope of the superconducting critical field curves and Rutger's formula, are also in close agreement with the BCS theory. Within the accuracy of the experimental data, the electronic specific heat coefficient, $Y$, remains essentially constant. 


\section{INTRODUCTION}

\section{A. Historical Survey}

Since the discovery of superconductivity by Kamerlingh Onnes (1) In 1911, the phenomenon has been the object of numerous experimental and theoretical investigations. only a few of the important experiments and calculations which bear directly on this work will be presented here. For more extensive reviews of the subject, the reader is referred elsewhere $(2-12)$.

One of the most easily observed properties of a superconductor is that below some transition temperature $T_{C^{\prime}}$ its electrical resistivity, $\rho$, is for all practical purposes, zero. Measurements have established an upper limit on the electrical resistivity in the superconducting state of about $10^{-23} \Omega-c m(13)$, a figure which may be compared to the lowest electrical resistivity measured in the normal state of metals of about $10^{-9} \Omega-c m(14)$. Another important property is that superconductivity may be destroyed by the application of a magnetic field at any temperature $T$ below $T_{C}$. Experimentally, it is found for all superconductors that the temperature dependence of this critical field, $H_{C}(T)$, is given approximately by

$$
\mathrm{h} \equiv \frac{\mathrm{H}_{\mathrm{C}}(\mathrm{T})}{\mathrm{H}_{\mathrm{C}}(0)} \simeq 1-\mathrm{t}^{2} \equiv 1-\left(\mathrm{T} / \mathrm{T}_{\mathrm{C}}\right)^{2} \text {. }
$$

In 1932 Keesom and Kok (15) found that in the absence 
of a magnetic field there is a jump in the electronic specific heat at the transition temperature characteristic of a second-order phase transition to a new thermodynamic phase (16). Keesom (17), Rutgers (18), and Gorter (19) applied thermodynamics to the superconducting transition, but the validity of these treatments was questioned because the phase transition was thought to be irreversible. This objection was removed, however, when Meissner and Ochsenfeld (20) determined that the magnetic induction, B, vanishes inside a bulk superconductor regardless of the magnetic field history of the sample. This phenomenon, known as the Meissner effect, showed that the superconducting state in an external magnetic field is a single stable state to which the laws of thermodynamics apply. Soon after the discovery of the Meissner effect, Gorter and Casimir (21) presented a full thermodynamic treatment of the superconducting phase transition.

The phenomenological two-fluid model of superconductivity was introduced by Gorter and casimir (22) in 1934. In this model it is assumed that a superconductor possesses an ordered or condensed state characterized by an order parameter which varies from zero at $T=T_{C}$ to unity at $T=0 \mathrm{~K}$, thereby indicating the fraction of the total system in the superconducting state. Further, it is assumed that the entire entropy of the system is due to the disorder of the particles 
in the normal state. The application of thermodynamics to the model yielded a parabolic critical field curve and an electronic specific heat varying as $\mathrm{T}^{3}$, both in general agreement with experiment. The electrodynamics of this model was worked out by F. and H. London (23) . The London theory predicts that the magnetic field does not abruptly disappear at the surface of a bulk superconducting specimen, but falls off exponentially with distance into the specimen, with a characteristic distance called the penetration depth, $\lambda$, a quantity first measured by. Shoenberg (24) in 1940. For most common superconductors $\lambda \sim 10^{-6} \mathrm{~cm}$ to $10^{-5} \mathrm{~cm}$.

The London model is based on a point by point relation between the current density and the vector potential associated with the magnetic field. This implies that the wavefunctions of the superconducting electrons are governed by. interactions which have a range much smaller than the penetration depth. On the basis of empirical evidence, however, Pippard (25) concluded that the current density, rather than being proportional to the vector potential at the point in question, is given by the spatial average of the vector potential over a region of extent $\xi \sim 10^{-4} \mathrm{~cm}$. Pippard called the quantity, $\xi$, the coherence length and found empirically that

$$
\xi=0.15\left({h v_{F}} / k_{B} T_{C}\right)
$$

where $h$ is Planck's constant divided by $2 \pi, v_{F}$ is the Fermi 
velocity, and $k_{B}$ is Boltzmann's constant. Microscopic theory confirms Equation 2 but the constant 0.15 is replaced by 0.18 . The superconducting state is now characterized by a long range coherence such that substantial changes in the superconducting wavefunctions cannot occur over a distance less than about $10^{-4} \mathrm{~cm}$ in pure metals. In a superconductor, then, there are two important characteristic lengths, $\lambda$ which determines the spatial variation of magnetic fields and $g$ which determines the spatial variations of the fraction of electrons in the superconducting ground state. Thus, we may classify superconductors as either type $I(\xi>\lambda)$ or type II $(g<\lambda)$. Because only type I materials will be considered in this thesis, no more will be said about type II behavior. The first success in understanding the origin of the superconducting state was made in 1950 when Fröhlich (26) noted that virtual emission and absorption of phonons by electrons could produce an attractive interaction between electrons. The importance of the electron-phonon interaction was soon verified experimentally by the discovery of the isotope effect (27) which showed that the transition temperature and isotopic mass, M, obeyed the approximate relation $\mathrm{T}_{c} \mathrm{M}^{1 / 2}=$ constant. An additional breakthrough came when improved thermal conductivity $(28)$ and specific heat measurements (29) and infrared absorption (30) measurements suggested the existence of an energy gap in the spectrum of the 
electrons in superconductors. About this same time; Cooper (3I) showed that any net attraction, no matter how small, between electrons can lead to a condensed state in which electron pairs are virtually excited above the Fermi surface. Finally, in 1957, a successful microscopic theory of superconductivity was presented by Bardeen, Cooper, and Schrieffer (32). This model, known as the BCS model, will be discussed in more detail later.

\section{B. Thermodynamic Theory of the Superconducting Transition}

Consider an ellipsoidal superconducting specimen of volume $\mathrm{V}$ with a magnetic moment $\mathrm{I}=\mathrm{MV}$, positioned in a solenoid such that the applied magnetic field $\mathrm{H}$ is parallel to the specimen's axis. All quantities will be expressed in cgs units. The relevant thermodynamic potential for the system is the Gibbs free energy

$$
\mathrm{G}=\mathrm{U}-\mathrm{TS}+\mathrm{PV}-\mathrm{HI},
$$

where $\mathrm{U}=$ internal energy, $\mathrm{T}=$ temperature, $\mathrm{S}=$ entropy, and $P=$ pressure. Differentiation of Equation 3 gives

$$
d G=d U-T d S-S d T+P d V+V d P-H d I-I d H=
$$

From the second law of thermodynamics

$$
\mathrm{dU}=\mathrm{TdS}-\mathrm{PdV}+\mathrm{HdI},
$$


where HaI is the work done on the sample by the solenoid's. power supply to change the sample's magnetic moment by $d I$. Substitution of Equation 5 into Equation 4 yields

$$
\mathrm{dG}=\mathrm{VdP} \cdot-\mathrm{SdT}-\mathrm{IdH} \text {. }
$$

Integration of Equation 6 gives

$$
G(T, P, H)=G(T, P, 0)-V \int_{0}^{H} M d H
$$

At $\mathrm{H}=\mathrm{H}_{\mathrm{C}^{\prime}}$ the superconducting state Gibbs energy, $\mathrm{G}_{\mathbf{S}^{\prime}}$ is equal to the normal state Gibbs free energy, $G_{n}$, so

$$
G_{S}\left(T, P, H_{C}\right)=G_{n}\left(T, P, H_{C}\right)
$$

The Gibbs free energy difference in zero field is then

$$
\Delta G=G_{n}(T, P, 0)-G_{S}(T, P, 0)=-V \int_{0}^{H}\left(M_{S}-M_{n}\right) d H,
$$

where $M_{S}$ and $M_{n}$ are the magnetizations of the superconducting and normal states, respectively.

Electromagnetic theory gives the relation

$$
B=H+4 \pi M \text {. }
$$

The Meissner effect shows that $B=0$ inside a superconductor, hence

$$
M_{S}=-H / 4 \pi .
$$


The normal state magnetization $M_{n}$ is negligible compared with $M_{S}$ and is taken to be zero. For a long cylindrical sample the demagnetization factor, D, is zero and the superconducting transition takes place at $\mathrm{H}_{\mathrm{C}} \cdot$ For an ellipsoidal sample with a different value of $D$, the field is distorted in the neighborhood of the sample and when the applied field reaches a value $(1-D) \mathrm{H}_{C^{\prime}}$ the sample goes into the intermediate state in which there is coexistence of macroscopic normal and superconducting regions. Regardless of the demagnetization factor,

$$
-V \int_{0}^{H} M_{S} d H=H_{C}^{2} / 8 \pi=\Delta G
$$

because the magnetic work is done solely in the superconducting portion of the sample.

The differences in the entropy and specific heat are given by

$$
\Delta S=S_{n}-S_{S}=-\frac{\partial}{\partial T}\left(G_{n}-G_{S}\right)_{P, H}=-\frac{{ }^{V H}}{4 \pi}\left(\partial H_{C} / \partial T\right)_{P}
$$

and

$$
\begin{aligned}
\Delta C & =C_{n}-C_{S}=T \frac{\partial}{\partial T}\left(S_{n}-S_{S}\right)_{P} . \\
& =-\frac{V T}{4 \pi} H_{C}\left(\partial^{2} H_{C} / \partial T^{2}\right)_{P}-\frac{V T}{4 \pi}\left(\partial H_{C} / \partial T\right)_{P}^{2},
\end{aligned}
$$

respectively. The third law of thermodynamics requires that 
$\Delta S=0$ at $T=0 \mathrm{~K}$, and Equation 13 shows that this requires $(\mathrm{\partial H} / \mathrm{OT})=0$ at $\mathrm{T}=0 \mathrm{~K}$. Further, $\Delta \mathrm{S}=0$ when $\mathrm{T}=\mathrm{T}_{\mathrm{C}}$ since $\mathrm{H}_{\mathrm{C}}=0$. Thus in zero field, the superconducting transition is accomplished without latent heat and is, therefore, a second-order phase transition. The specific heat jump at $\mathrm{T}_{\mathrm{C}}$ is given by Rutger's formula (16)

$$
\Delta C\left(T_{c}\right)=-\frac{V T}{4 \pi}\left[\partial H_{C}\left(T_{C}\right) / \partial T\right]_{P}^{2}
$$

on the basis of theoretical and experimental evidence the following assumptions are made concerning the entropy of a superconductor (33):

(i) The entropies in the normal and superconducting states may be expressed as the sums of the independent lattice $\left(s_{g}\right)$ and electronic $\left(s_{e}\right)$ contributions (i.e., $s_{n}=s_{g n}+s_{\text {en }}$ and $\left.s_{s}=s_{g s}+s_{e s}\right)$.

(ii) For sufficiently low. T, $S_{\text {en }} \gg S_{\text {es }}$.

(iii) The normal electron entropy is given by the free electron model result (i.e., $S_{e n}(T)=\gamma^{T}$ ).

(iv) The lattice entropy is unchanged in the superconducting transition (i.e., $s_{g n}=s_{g s}$ ).

Equation 13 now becomes 


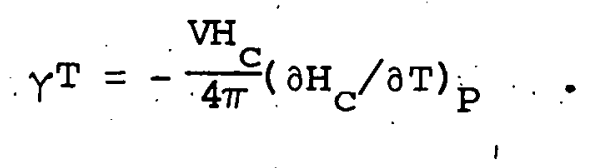

Integration of Equation 16 from 0 to $T$ gives the important result

$$
\mathrm{H}_{\mathrm{C}}^{2}=\mathrm{H}_{0}^{2}-4 \pi(\gamma / \mathrm{V}) \mathrm{T}^{2}
$$

where $\mathrm{H}_{\mathrm{O}}=\mathrm{H}_{\mathrm{C}}(0)$. The BCS theory predicts that Equation 17 is valid below $t=0.25$, a result that has been experimentally verified (33). Thus critical field measurements at sufficiently low temperatures allow the determination of both $\mathrm{H}_{0}$ and $\gamma$

\section{BCS Microscopic Theory of Superconductivity}

The BCS microscopic theory of superconductivity was successful in explaining the principal experimental facts of superconductivity discussed previously: (1) a secondorder phase transition at $T_{C^{\prime}}$ (2) zero resistivity; (3) the Meissner effect; (4) an energy gap for single particle excitations; and (5) the isotope effect.

BCS assumes that in a metal there is an attractive force between electron pairs caused by virtual phonon exchange and a repulsive force due to the screened coulomb interaction and when the combined effect of the two forces is attractive, the metal becomes superconducting. Because superconductivity is fairly common in metals and is not 
associated with any particular crystal structure, the net attractive interaction is assumed isotropic and is replaced by a constant attractive interaction $-V^{\prime}$ within a characteristic energy thw of the Fermi surface. The normal state is described by the Bloch individual-particle model while the superconducting ground state is formed by taking a linear combination of many low-lying normal state configurations in which the Bloch states are virtually occupied in pairs of opposite spin and momentum $(\vec{k} \uparrow,-\vec{k} \downarrow)$. These paired states are referred to as Cooper pairs. The transition temperature is related to the characteristic energy, $\hbar w$, the density of electron states for one spin direction at the Fermi energy, $N(0)=N\left(\varepsilon_{F}\right)$, and the attractive interaction, $-V^{\prime}$, in the weak coupling limit $N(0) V^{\prime}<<1$ by the relation

$$
k_{B} T_{C}=1.14 \not h \omega \exp \left[-1 / N(0) v^{\prime}\right]
$$

Since the most attractive part of the interaction is due to high frequency phonons, hw is expected to be of the order of $k_{B} \theta_{D}$, where $\theta_{D}$ is the Debye temperature. In actual practice $h \omega$ is assumed to be approximately 0.75 hw (34), so Equation 18 is usually seen in the form

$$
{ }^{-} T_{C}=0.85 \theta_{D} \exp \left[-1 / N(0) V^{\prime}\right]
$$

The Gibbs free energy difference at $\mathrm{T}=0 \mathrm{~K}$ is 


$$
\Delta_{G} / V=1 / 2 N(0)[\Delta(0)]^{2},
$$

where $2 \Delta(0)$ is the energy gap at $T=0 \mathrm{~K}$. Using the free electron model's result for the electronic specific heat coefficient $Y$,

$$
Y=\frac{2}{3} \pi^{2} k_{B}^{2} N(0) v \text {, }
$$

and Equation 12 we get

$$
\Delta(0)=\mathrm{H}_{0}\left(\pi k_{B}^{2} \mathrm{~V} / 6 \gamma\right)^{1 / 2} .
$$

Mühlschlegel (35) calculated the thermodynamic functions using the BCS theory in the weak coupling limit and expressed the results in the following form

$$
\begin{aligned}
& \left(\mathrm{VH}_{\mathrm{c}}^{2} / 8 \mathrm{~T}\right) / \gamma_{\mathrm{T}} \mathrm{T}_{\mathrm{c}}^{2}=\frac{3}{2} t^{2}\left(\mathrm{x} \frac{\mathrm{da}}{\mathrm{dx}}-\mathrm{a}\right), \\
& s_{e s} / \gamma^{T} c=t\left[i+3\left(x \frac{d a}{d x}-a\right)-\frac{3 x}{2}\right],
\end{aligned}
$$

where

$$
\begin{aligned}
& \mathbf{x}=\left[\Delta(T) / \pi k_{B} T\right]^{2}, \\
& 2 \Delta(T)=\text { energy gap at temperature } T, \\
& \Delta(0)=\frac{\pi}{Y_{e}} k_{B} T_{C}=1.764 k_{B} T_{C}, \\
& \ln \gamma_{e}=\text { Euler's constant, }
\end{aligned}
$$


and

$$
\begin{aligned}
a(x)= & -\frac{2}{\pi} \int_{-\infty}^{\infty} \ln \left(1+e^{-\pi\left(x+u^{2}\right)^{1 / 2}}\right) d u \\
& +x\left(\ln \gamma_{e} \sqrt{x}-1 / 2\right)+1 / 3
\end{aligned}
$$

The BCS critical field calculations agree remarkably well with experimental data for pure superconductors. Deviations. from BCS in pure superconductors are primarily due to strongcoupling effects, exhibited by $\mathrm{Hg}$ (33) and $\mathrm{Pb}$ (36), for example, and anisotropy effects, exhibited in Al (37), for example. Strong-coupling theory $(38,39)$, which uses the actual phonon spectrum of the superconductor to calculate the electron-phonon interaction matrix element, has improved agreement between theory and critical field data of $\mathrm{Hg}$ and $\mathrm{Pb}$. Clem (40) modified the critical field calculation by including an anisotropic form of the electron-phonon interaction matrix element and this theory has explained a number of experiments, notably the effects of alloying on superconductivity and the deviation of low $\mathrm{T}_{\mathrm{C}}$ superconductors from BCS. 


\section{Addition of Impurities to a Pure Metal}

The propertles of both normal and superconducting metals containing small concentrations of impurities with partialiy filled d- or f-electron shells have been the subject of extensive experimental and theoretical investigations. An isolated atom with unfilled d or f states generally has a spin and orbital angular momentum according to Hund's rules, and it frequently happens that the perturbations acting in a metal on an impurity are of insufficient strength to destroy the magnetic moment. A typical example of such local moment behavior occurs when atoms of $3 d$ transition elements are dissolved in noble metals. In such a case, the scattering of the conduction electrons may depend upon the relative orientation of the spins of the impurity atoms and, in addition, both potential and spin-flip scattering may occur. These scatterings can give rise to various anomalous properties, such as the appearance of a Kondo term

$$
\rho \propto-\operatorname{An} \ln T
$$

in the low temperature electrical resistivity for an impurity concentration $\mathrm{n}$, a giant thermoelectric power, and other related phenomena. A number of excellent review articles have treated the local moment problem for both normal (41-44) and superconducting (45-51) metals, and for more detailed discussions of the problem, the reader is 
referred to these reviews and the references cited therein. J. Friedel (52) first gave an explanation of the local moment in terms of the concept of the "virtual bound state". The basic idea of his theory is that one has an element I with a partially filled $\ell$ band ( $\ell$ denotes $d$ or $f$ ) dissolved in a normal metal matrix $M$, and assumes that $I$ has an atomic energy level which lies within the bandwidth of the $s$ conduction electrons (Figure 1 ). The $\ell$ radial wavefunction presumably does not extend very far out of the I atomic cell, so it will retain some of its atomic behavior in the alloy. Because the energy of the $l$ radial wavefunction is higher than the bottom of the conduction band, it gets broadened by mixing with nearby free electron states having approximately the same energy in the conduction band. If the broadening is not too great, the electronic charge of I remains strongly localized in a virtual $l$-bound state in order to keep the ionic charge of I equal to that of M. Another way of looking at the problem is to reduce the interaction to an equivalent scattering problem in which I gives up all its $s$ and $\ell$ electrons to the conduction band of $\mathrm{M}$, thereby creating a strong localized Coulomb potential well whlch is almost deep enough to have bound $l$ states for every $l$ electron given up. As a result, the $l$ components of the conduction electron wave functions are strongly attracted to the atomic cell of I when their energy is near that of the vacated $l$ levels. One 


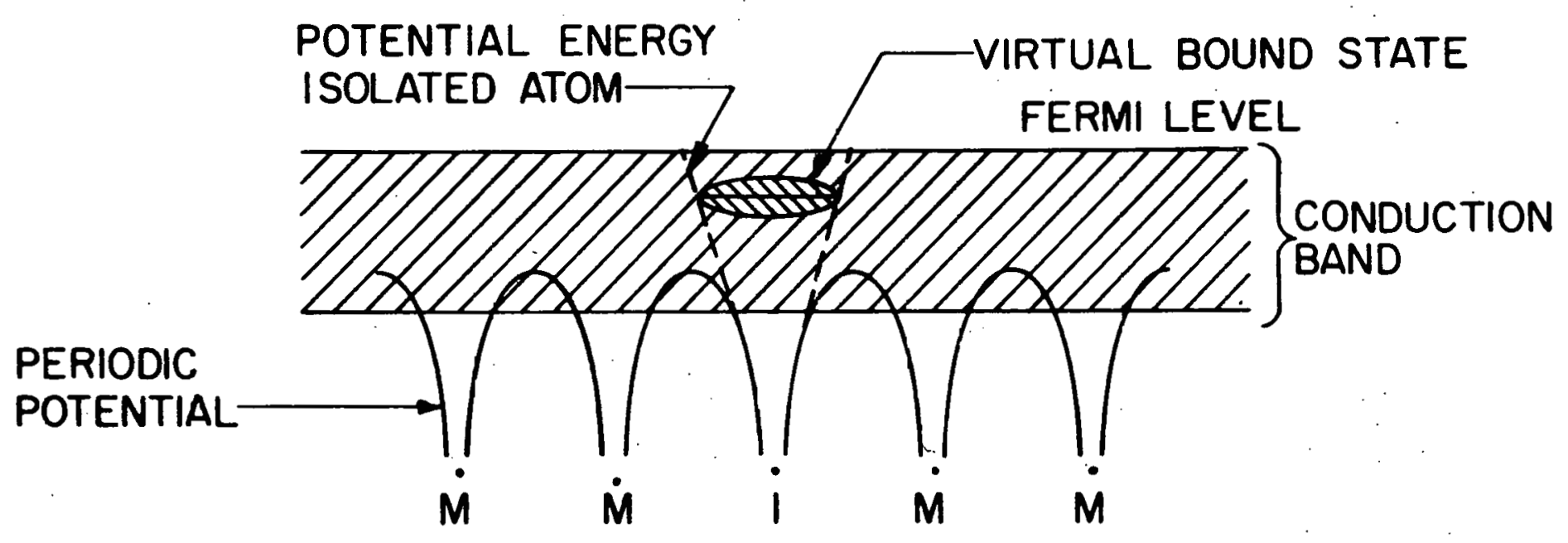

Figure 1. Virtual bound $l$-state on an impurity atom $I$ in a normal metal matrix $M$. 
still has a virtual bound state localized on $I$, which appears here as a resonance in the potential of the I atomic cell.

In order to explain the presence of a net magnetization on I, Friedel pointed out that since the virtual bound states are almost as much localized as in an atom, one would expect exchange and correlation forces to be very effective for narrow bands, with a tendency to favor spin alignment. When these forces prevail the virtual bound states have different energies for opposite spin alignment so that they accommodate unequal numbers of electrons up to the Fermi level and a localized magnetic moment exists.

In 1961, Anderson (53) put these ideas on a more quantitative basis by solving the following problem in the Hartree-Fock approximation at $\mathrm{T}=0 \mathrm{~K}$. Assume that a localized moment exists (Figure $2 a$ ) and can be represented by a single $\ell$-orbital level whose energy is a distance $E_{\ell}=\mathrm{E}$ below the Fermi level, $\varepsilon_{F^{\prime}}$ so that it is occupied by an electron of say, spin-up. A spin-down electron attempting to occupy the same orbital will see the full Coulomb repulsion, $\mathrm{V}_{\mathrm{C}^{\prime}}$ of the spin-up state because the exclusion principle allows the electrons to get relatively close to one another. The spin-down electron can only occupy an orbital with energy $E+V_{c^{\prime}}$ which must be empty if a local moment exists and, therefore, must lie above the Fermi level. Now, as before, 


\section{(a) MAGNETIC}

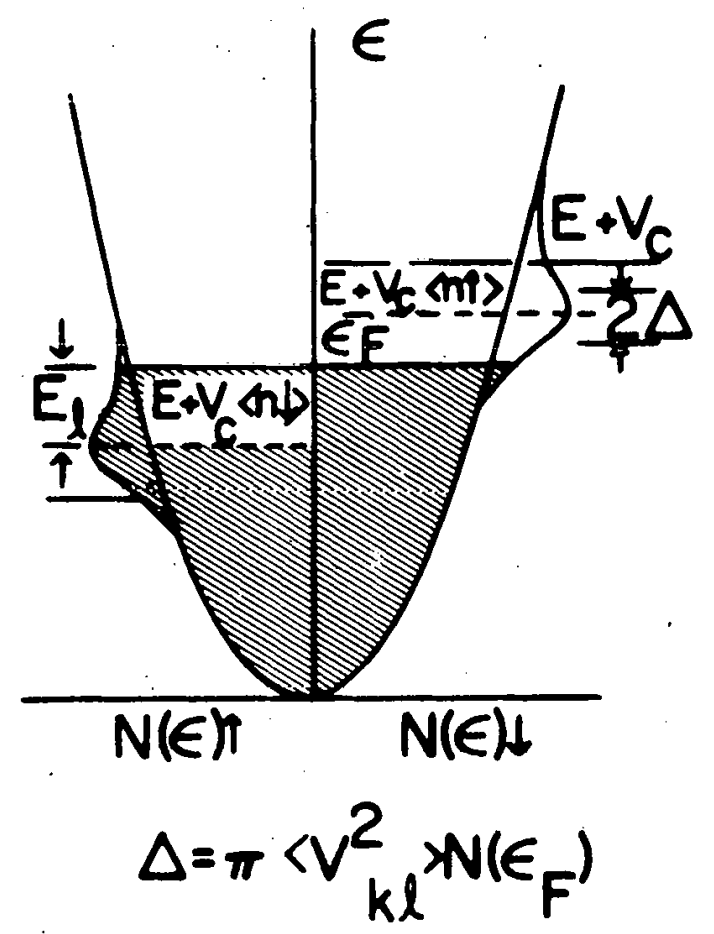

(b) NONMAGNETIC

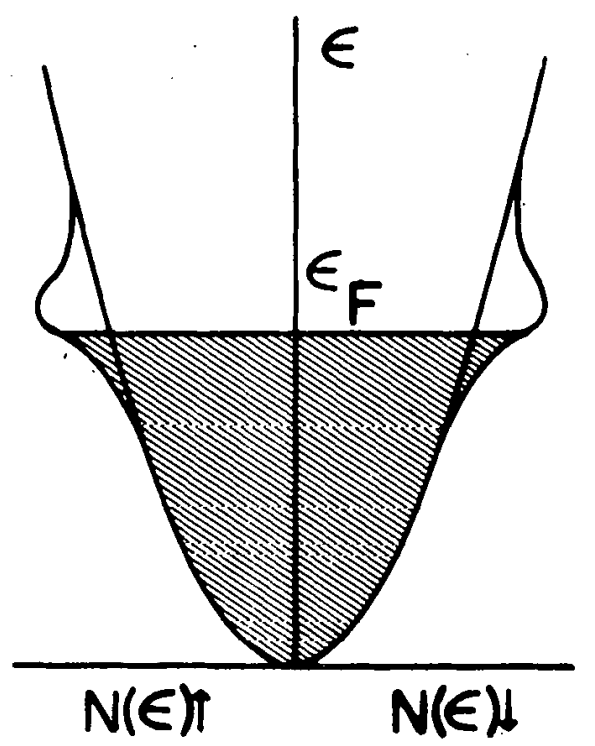

Figure 2. Anderson model density of states distributions in (a) magnetic and (b) nonmagnetic cases. 
the conduction electrons can, through the mixing interaction, $\mathrm{V}_{k \ell^{\prime}}$ mix with the electron in the localized state and cause that level and the empty level above $\varepsilon_{F}$ to be shifted and broadened with half-width $\Delta$. The expectation value $\langle$ n $\uparrow\rangle$ now becomes less than unity, while the expectation value $\langle n \downarrow\rangle$ becomes finite. These changes are such as to decrease the difference between the spin-up and spin-down energies: $\mathrm{E}$ moves up to $E+\langle n \downarrow\rangle V_{C}$ and $E+v_{C}$ moves down to $E+\langle n \uparrow\rangle v_{C}$. If $\mathrm{v}_{\mathrm{k} \ell}$ is strong enough, the situation eventually breaks down cooperatively and the system becomes unstable so that it is no longer possible to maintain a localized moment and we have a nonmagnetic state (Figure $2 \mathrm{~b}$ ). Anderson (53) formulated a Hamlitonian for this model and the solutions of this Hamiltonian allowed him to define the transition curve, shown in Figure 3, separating magnetic and nonmagnetic regions. An examination of Figure 3 reveals that in the Anderson model the magnetic regime exists for $v_{C} \gg \Delta$ when $\left(\varepsilon_{F}-E\right) / v_{C}$ is neither too small nor too near unity, while the nonmagnetic regime exists for either $v_{C} \ll \Delta$ or, when $\left(\varepsilon_{F}-E\right) / v_{C}$ is close to 0.5 for relevant values of $v_{C} / \Delta$. The Anderson model can be generalized to the case where there are $2(2 l+1)$ degenerate llevels, which fill to the Ferml level with approximately the number $\langle\mathrm{N}\rangle$, of l-electrons appropriate for the impurity being considered. Taking the Fermi level as the zero of energy, the level centers are separated from it by an energy 


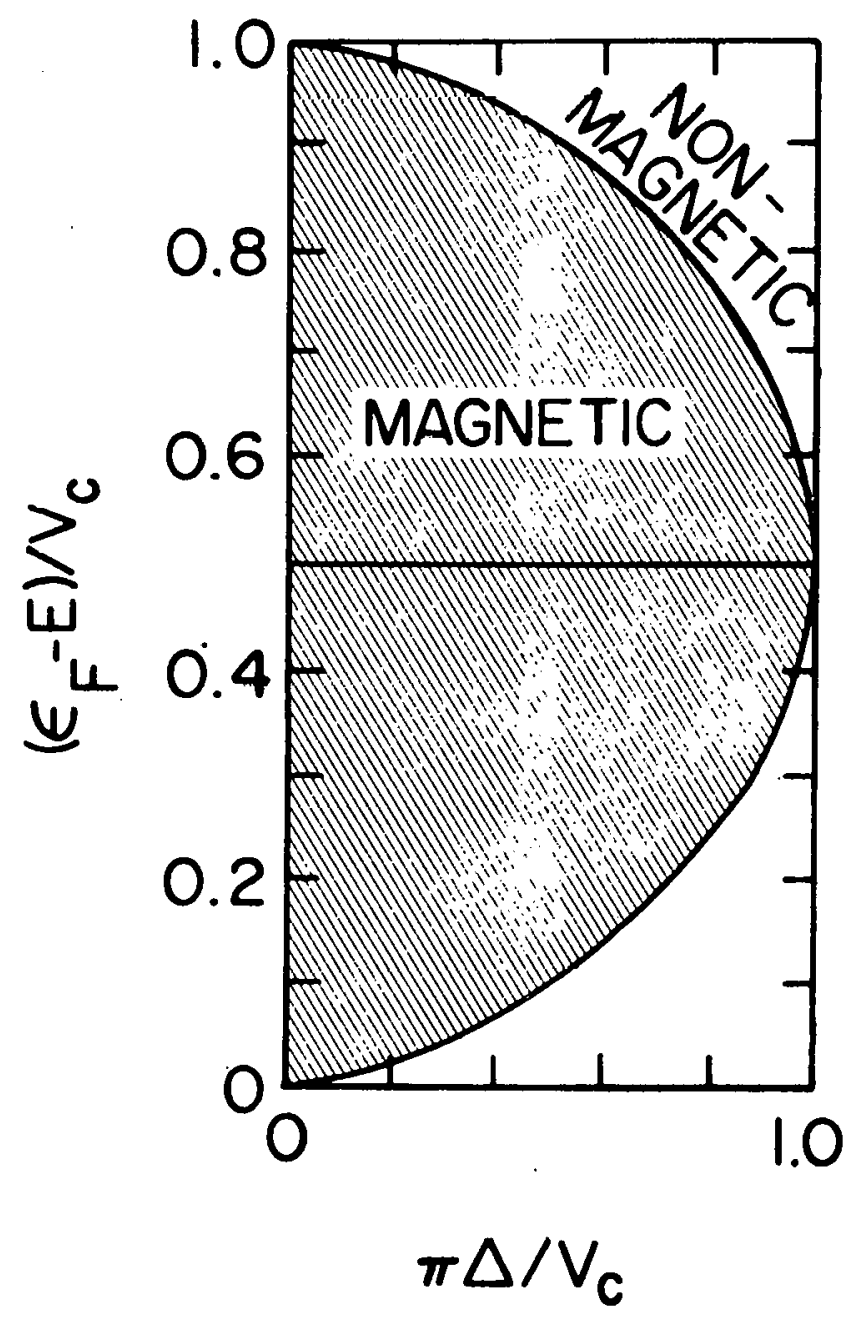

Figure 3. Anderson model magnetic-nonmagnetic phase diagram. 
${ }^{E} l$ which depends on $\langle\mathrm{N}\rangle$ and the level width, $\Delta$, according to

$$
E_{l}=\Delta \cot [\pi\langle\mathrm{N}\rangle / 2(2 \ell+1)] \text {. }
$$

where

$$
\Delta=\pi N(0)\left\langle\mathrm{v}_{\mathrm{k} \ell}^{2}\right\rangle
$$

For a single level, the l-electron density of states $\mathrm{N}_{\ell}(0)$ is

$$
\mathrm{N}_{\ell}(0)=\Delta / \pi\left(E_{l}^{2}+\Delta^{2}\right) \text {. }
$$

and the magnetic-nonmagnetic phase boundary is defined by the relation

$$
\left.\sin ^{2}[\pi<\mathrm{N}\rangle / 2(2 \ell+1)\right]=\pi \Delta / \mathrm{V}_{\mathrm{C}}
$$

Kondo (54) gave the first theoretical explanation of the resistance minimum which appears in the low temperature electrical resistivity of metals containing magnetic impurities (hence the name Kondo effect). He assumed that a local moment exists and characterized the impurity by assigning it a spin $\vec{S}$ which interacts with the conduction electrons through an exchange interaction represented by the interaction Hamiltonian

$$
\text { Hint. }=-2 \mathrm{~J} \overrightarrow{\mathrm{s}} \cdot \overrightarrow{\mathrm{s}} \text {, }
$$

where $J$ is the strength of the exchange interaction and $\vec{s}$ 
is the average conduction electron spin density at the impurity site. A pertubation calculation of the conduction electron-impurity scattering to third order in $J$ gave, for $J<0$, a magnetic contribution to the electrical resistivity proportional to $-\ln \mathrm{T}$, in agreement with experiment, but the expression diverged for negative $J$ below a characteristic temperature of

$$
T_{K} \sim T_{F} \exp [-1 / N(0)|J|],
$$

where $T_{F}=\varepsilon_{F} / k_{B}$ is the Fermi temperature. Subsequent applications of more sophisticated techniques to try to remove the divergence and to explain properties for temperatures both above and below the Kondo temperature, $\mathrm{T}_{\mathrm{K}}$ ' has led to the physical interpretation of $\mathrm{T}_{\mathrm{K}}$ as a characteristic temperature below which the impurity spins tend to be compensated by the conduction electron spins, the degree of compensation increasing with decreasing temperature.

A crucial factor in all these theories is the sign and magnitude of the exchange interaction parameter, $J$, so it is important to understand the factors which determine these quantities. The ordinary atomic exchange $J_{0}$ is always positive, but, as Anderson and clogston (55) first pointed out, the Anderson type mixing can lead to a negative term for $\mathrm{J}$. Indeed, Schrieffer and Wolff (56) performed a canonical transformation on the Anderson Hamiltonian and found that 
in the limit of small mixing between the local moment and conduction electron states, the Anderson Hamiltonian became identical to the Kondo Hamiltonian. Generally, $J$ is given approximately by

$$
J-J_{0}+J_{1},
$$

where $J_{0}(>0)$ is the atomic exchange term and $J_{1}(<0)$ arises from the mixing between the conduction electrons and local impurity states. For transition metal solutes in noble metal matrices the dominant contribution to $J$ is often $J_{i}$ and $\mathrm{J}<0$, leading to Kondo type behavior, while for rare earth solutes, with the exception of $\mathrm{Ce}, \mathrm{J}_{0}$ is the dominant contribution to $J$, hence the absence of the Kondo effect. In terms of the Schrieffer-Wolff transformation $J_{1}$ is given by

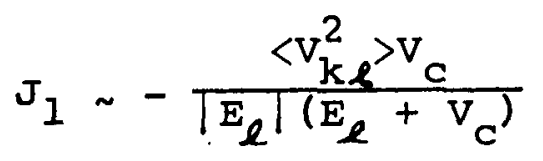

when $\left|E_{\ell}\right| \gg \Delta$. Here $E_{\ell}$ is the energy separating the local state and the Fermi level.

The Hartee-Fock approximation used by Anderson in his model excludes the dynamics of the problem. The operational definition of the existence of a local moment is that the excess susceptibility of the alloy over that of the pure host approximates a Curie law behavior, at least above some 
temperature. This requires that the spin polarization on the impurity be long-lived, but not infinitely long-lived as in Anderson's model. Extensions of the theory to include time-dependent Hartree-Fock approximations have led to the development of a localized spin fluctuation (Isf) model (57). According to the lsf model, even when a system is nonmagnetic in the Anderson sense $\left(v_{C}<\pi_{\Delta}\right)$, the system can suffer large fluctuations in spin density. Physically, we might view this as an electron hopping onto the impurity state and remaining there for some characteristic lsf time, $\tau_{s f} \sim h / \Delta$, before hopping off. Associated with $\tau_{\text {sf }}$ is a characteristic temperature $T_{0} \sim \mathrm{h} / \mathrm{k}_{\mathrm{B}} \tau_{\mathrm{Sf}}$. In the limit $\tau_{\mathrm{Sf}} \gg \tau_{\mathrm{T}^{\prime}}$ where $\tau_{\mathrm{T}}$ is the thermal fluctuation lifetime $\left(\sim \mathrm{h} / \mathrm{k}_{\mathrm{B}} \mathrm{T}\right)$, the impurities behave magnetically, while in the limit $\tau_{S f} \ll \tau_{T^{\prime}}$ the impurities behave nonmagnetically. This is equivalent to saying that when the temperature becomes of the order $\mathrm{T}_{0}$, the lsf becomes stable against thermally induced reorientation, and, at this temperature and above, there is no physical difference between the spin fluctuation and a genuine spin. Hence, the system behaves as if it were magnetic. For this theory the low temperature electrical resistivity is predicted to follow a $\rho_{0}\left[1-\left(T / T_{0}\right)^{2}\right]$ behavior. Such behavior was first observed in AlMn and AlCr by Caplin and Rizzuto (58).

The local moment problem is considerably more complicated 
if the impurity host undergoes a superconducting transition. When the impurities are nonmagnetic atoms with filled d- or f- shells;, the superconducting transition temperature is changed by at most a few percent, as first found in studies using $S n$ (59), and AI and In (60), as superconducting hosts. For sufficiently small concentrations of all solutes these studies showed that $T_{C}$ decreased linearly with increasing reciprocal electronic mean free path, $1_{e} \cdot$ For larger impurity concentrations, the curves of $\mathrm{T}_{\mathrm{C}}$ versus concentration fell into two groups, according to the sign of the valence difference, $\Delta \mathrm{Z}$, between the impurity and host. . T had a sharp upward curvature for higher valence solutes, while for those with lower valence, $T_{C}$ tended to saturate for large concentration. This behavior was in sharp contrast to the rapid depression of $T_{C}$ which had been observed in superconductors containing 3d-transition (61) and 4f-rare earth (62) magnetic impurities, where the addition of a few tenths of atomic percent of impurity sometimes completely suppressed superconductivity. The small depression of $T_{c}$ in the nonmagnetic impurity case was difficult to understand within the framework of the BCS microscopic theory of superconductivity for the following reason. In the BCs theory it was shown that a net attractive interaction using timereversed Bloch states led to the formation of Cooper pairs which have a binding energy of about $10^{-4} \mathrm{eV}$ at $\mathrm{T}=0 \mathrm{~K}$. In 
very "dirty" samples where $l_{e} \sim \xi$, the collision time $\tau$ is of the order of $10^{-14}$ sec, corresponding to an energy uncertainty of about $0.1 \mathrm{eV}$ and all superconducting effects would be expected to change drastically, contrary to the experimental result.

Anderson's theory of dirty superconductors (63) provided an explanation of the experimental results discussed above. He suggested that the anisotropy of the energy gap present in the pure superconductor may be smoothed out by the impurity scattering. In the pure metal, the energy gap is anisotropic, depending on the momentum direction of the electrons, and $T_{C}$ is primarily dependent on the large gap values. The impurity scattering mixes Bloch states of the conduction band. Therefore, the energy gap and $T_{c}$ will decrease with decreasing $1_{e}$, until, when $l_{e} \sim g$, the impurity scattering becomes so large that Bloch states from all parts of the Fermi surface are mixed with more or less equal weight, thereby removing the source of anisotropy in the energy gap. Once the mixing is complete, the effect of $I_{e}$ on $T_{C}$ saturates. The formation of cooper pairs then takes place between the time-reversed states which are, exact one electron states of the impure metal. Anderson showed that if the scatterers are nonmagnetic, the time-reversed states are degenerate in energy and lead to the formation of cooper pairs with infinite lifetimes as in BCS theory. If the 
impurities are magnetic, however, time-reversal invariance is removed, the time-reversed scattered states are no longer degenerate in energy, and the Cooper pairs now have finite lifetimes. Such a pair-breaking interaction inhibits superconducting electron correlation and large decreases in $\mathrm{T}_{\mathrm{C}}$ occur.

The basic features of the $\mathrm{T}_{\mathrm{C}}$ versus concentration $\left[T_{C}(n)\right]$ studies of La containing rare earth impurities by Matthias et al. (62) were successfully explained by Abrikosov and Gor'kov (64) in 1960. The Abrikosov-Gor'kov (AG) theory makes three basic assumptions: (1) the paramagnetic impurity spins $\vec{S}$ are randomly distributed on the host lattice; (2) the impurity spins are unpolarized and uncorrelated; and (3) an impurity spin interacts with the average electron spin density at the impurity site via the exchange interaction given by Equation 33. The presence of the paramagnetic impurities leads to the formation of Cooper pairs with a finite lifetime $\tau$. Within the first Born approximation, the inverse lifetime $\tau^{-1}$ (or pair-breaking parameter $\alpha$ ) is given by

$$
\alpha \equiv \tau^{-1}=\not^{-1} \mathrm{nN}(0) \mathrm{J}^{2} \mathrm{~S}(\mathrm{~S}+1) \text {, }
$$

where $\mathrm{n}$ is the impurity concentration. For rare earth impurities where the orbital angular momentum is not quenched, $\vec{s}$ must be replaced by its projection on the total angular 
momentum vector $\vec{j}(65)$. In this case, Equation 33 becomes

$$
\psi_{\text {int. }}=-2 J(g-1) \vec{j} \cdot \vec{s} \text {, }
$$

where $g$ is the Lande g-factor, and Equation 37 becomes

$$
\alpha \equiv \tau^{-1}=\not^{-1} \mathrm{nN}(0) \mathrm{J}(g-1)^{2} j(j+1)
$$

The theory predicts a second-order phase transition to the superconducting state in the absence of a magnetic field, and connects the reduced temperature $T_{C} / T_{C P}$ to the reduced pair-breaking parameter $\alpha / \alpha_{\text {cr }}$ through the relation

$$
\ln \left(T_{c} / T_{c p}\right)=\psi(1 / 2)-\psi\left(1 / 2+\frac{1}{4 \gamma_{e}} \frac{\alpha}{\alpha_{c r}} \frac{T_{c p}}{T_{c}}\right) .
$$

Here $\psi$ is the digamma function, $T_{C p}$ and $T_{C}$ are the superconducting transition temperatures of the pure host and alloy, respectively, and $\alpha_{\mathrm{cr}} \equiv \mathrm{k}_{\mathrm{B}} \mathrm{T}_{\mathrm{cp}} / 4 \hbar_{\gamma_{\mathrm{e}}}$ is the critical value of the pair-breaking parameter required to destroy superconductivity. In the limit of small $\alpha / \alpha_{\mathrm{cr}}$. Equation 40 has the asymptotic form

$$
\lim _{\frac{\alpha}{a_{c r}} \rightarrow 0} \cdot T_{c} / T_{c p}=1-0.691\left(\alpha / \alpha_{c r}\right)
$$

If $\alpha$ is varied only by changing the impurity concentration $n$, then $\alpha / \alpha_{c r}$ in Equations 40 and 41 may be replaced by $\mathrm{n} / \mathrm{n}_{\mathrm{Cr}}$, where $\mathrm{n}_{\mathrm{cr}}$ is the critical concentration at which 
$T_{c}=0$.

The AG theory also made the remarkable prediction that for an impurity concentration greater than $0.91 \mathrm{n}_{\mathrm{cr}}$, the energy gap in the single particle density of states disappears and the phenomenon of gapless superconductivity occurs. This results from the fact that the finite cooper lifetime $\tau$ leads to an energy broadening $\Gamma \sim \mathrm{h} / \tau$ which introduces states into the gap and spreads out the BCS density of states. As a consequence of this energy broadening, the energy gap goes to zero faster than the superconducting order parameter. This leads to a specific heat which varies linearly with temperature rather than exponentially in the gapless region.

There exists a large body of experimental evidence which verifies the correctness of the AG theory and its extensions: Skalski et al. (66) have calculated the density of states, critical field, discontinuity in the specific heat at $\mathrm{T}_{\mathrm{C}^{\prime}}$ penetration depth, and complex conductivity; and Ambegaokar and Griffin (67) have calculated the thermal conductivity for an AG material. A few of the more relevant experiments confirming the theory are the following: tunneling measurements on PbGd by Wolff and Reif (68): specific heat measurements on bulk LaGd alloys by Finnemore et al. (69): tunneling measurements on $S n$ and $S n-I n$ alloys by Millstein and Tinkham (70); critical field measurements on 
ThGd by Decker et al. (71) and Decker and Finnemore (72); $\mathrm{T}_{\mathrm{C}}(\mathrm{n})$ measurements to $\mathrm{n}=0.9 \mathrm{n}_{\mathrm{Cr}}$ on $(\underline{\mathrm{La}}, \mathrm{Gd}) \mathrm{Al}_{2}$. by Maple (73) which are show in Figure 4; thermal conductivity measurements on ThGd by Cappeiletti and Finnemore (74); and far-infrared measurements on PbGd by Dick and Reif (75).

Large departures from the AG $T_{c}(n)$ curves have been observed for $\dot{n}>0.7 \mathrm{n}_{\mathrm{Cr}}$ for LaGd (76) and (La,Gd) ${ }_{3}$ In (77), and Bennemann (78) has attributed these departures to the magnetic ordering of impurity spins. According to Bennemann's theory, the magnetic ordering of the impurity spins leads to three effects: (1) zeeman splitting of the Fermi surface, which is destructive to superconductivity; (2) reduction of spin-flip scattering which enhances superconductivity; and (3) spin-orbit scattering, which reduces the effect of the zeeman splitting of the Fermi surface and, therefore, enhances superconductivity. By considering the interplay of these three effects, Bennemann was able to show qualitative agreement with the $T_{C}(n)$ curves for LaGd and $(\underline{L a}, G d)_{3}$ In. Additional corrections to the AG critical field curves to include additional pair-breaking effects have been given by Fulde and Maki (79) and Bennemann, Garland, and Mueller (80). Since these corrections deal with type II superconductors, the reader is referred to these papers and to the review articles by Parks (49), Bennemann (50), and Maki (51) for further discussion. 


\section{THIS PAGE}

\section{WAS INTENTIONALLY \\ LEFT BLANK}


Figure 4. Reduced transition temperature $\mathrm{T}_{\mathrm{c}} / \mathrm{T}_{\mathrm{cp}}$ versus reduced concentration $\mathrm{n} / \mathrm{n}$ cr for ( $\underline{\mathrm{La}}, \mathrm{Gd}) \mathrm{Al}_{2}$ from Maple (73), where $\mathrm{T}_{\mathrm{cp}}=3.24 \mathrm{~K}$ and $\mathrm{n}_{\mathrm{cr}}=0.590$ at.\% Gd. The solid curve represents the result of the AG theory (Equation 40). 


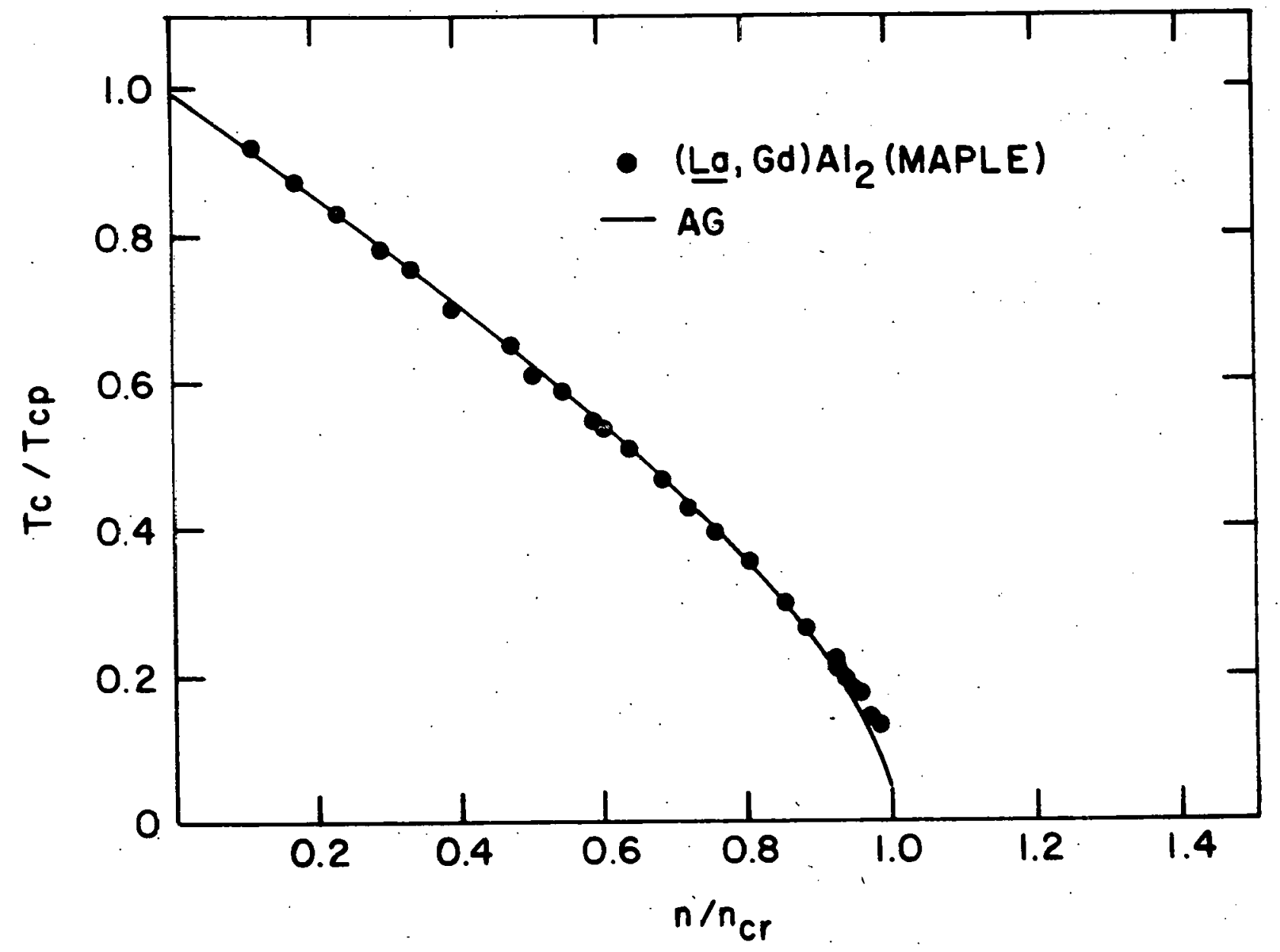


Recently, the problem of paramagnetic impurities in superconductors has been reinvestigated by including the Kondo effect. According to Müller-Hartmann and zittartz (81) and.Ludwig and Zuckermann (82), Equation 40 still describes a superconducting Kondo system if the pairbreaking parameter is given by

$$
a=\frac{n}{h(2 \pi)^{2} N(0)} \frac{\pi^{2} S(S+1)}{\ln ^{2} T_{C} / T_{K}+\pi^{2} S(S+1)},
$$

which has a maximum at $\mathrm{T}_{\mathrm{K}}$ given by Equation $34 \cdot \mathrm{T}_{\mathrm{C}}(\mathrm{n})$ can then be calculated by simultaneously solving Equations 40 and 42. Several examples are shown in Figure 5 from the work of Ludwig and Zuckermann (82) for $T_{C p}=9.17 \mathrm{~K}$ and $S=1 / 2$. For $T_{K} \geq T_{C p}$. (curves $A$ and $C$ ), $T_{C}(n)$ initially decreases linearly with increasing $n$ after which it curves positively away from linearity in contrast to the AG result. A particularly interesting result can occur when $\mathrm{T}_{\mathrm{K}} \ll \mathrm{T}_{\mathrm{CP}}$ (curve $\mathrm{B}$ ). For certain values of $\mathrm{n}$, Equations 40 and 42 yield three solutions for $T_{C}(n)$. As the temperature is lowered, an alloy of the appropriate composition should first become superconducting at a temperature $T_{C l}(n)$, remain superconducting to a lower temperature $T_{c 2}(n)$ at which it becomes normal, and then remain normal to yet a lower temperature $\mathrm{T}_{\mathrm{C} 3}(\mathrm{n})$ at which it again becomes superconducting. The above behavior is understandable in view of the temperature 


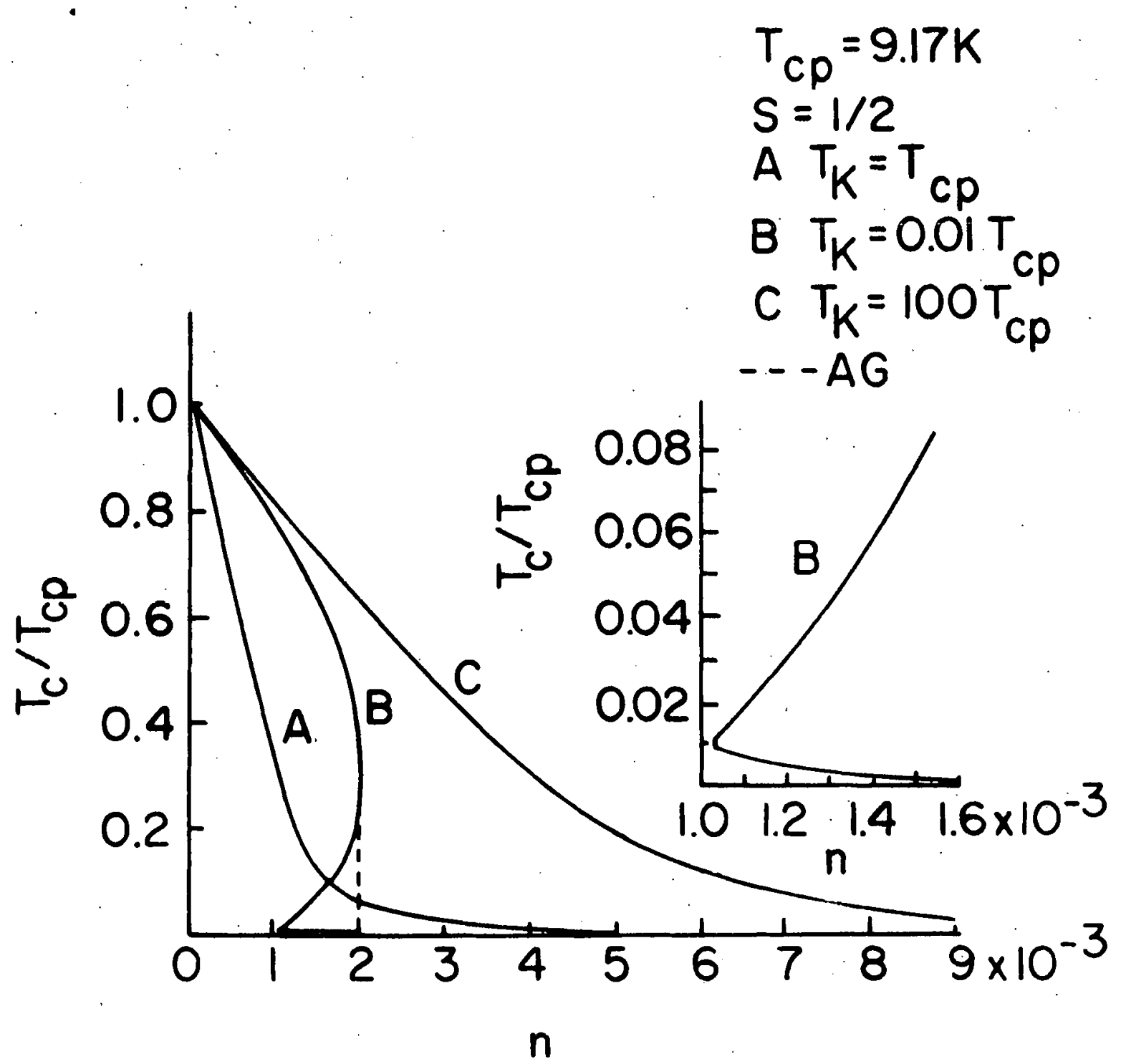

Figure 5. $\mathrm{T}_{c} / \mathrm{T}_{\mathrm{cp}}$ versus impurity concentration $\mathrm{n}$ for various values of $\mathrm{T}_{\mathrm{CP}} / \mathrm{T}_{\mathrm{K}}$ from Ludwig and Zuckermann (82). 
dependent pair-breaking given by Equation 42 . For $T_{C p} \gg T_{K^{\prime}}$ the alloy becomes superconducting at an upper transition temperature where the pair-breaking is still small. When the temperature is lowered, pair-breaking increases strongly as $\mathrm{T}_{\mathrm{K}}$ is approached, and superconductivity is again suppressed. Superconductivity then reappears for $T<T_{K^{\prime}}$ where the temperature is much less than the resonant temperature.

A reentrant $T_{C}(n)$ curve has been observed in the (La, Ce) $\mathrm{Al}_{2}$ system $(83,84)$, where $\mathrm{T}_{\mathrm{K}} \ll \mathrm{T}_{\mathrm{CP}}$, but no evidence was found for a third transition temperature even though measurements were extended to $0.006 \mathrm{~K}$. The extension of the Kondo effect by Maki (85) to type II superconductors is roungly in agreement with critical field curve measurements of this alloy system (86). Also, the specific heat jump at $\mathrm{T}_{\mathrm{C}}$ determined from specific heat measurements on ( $\left.\mathrm{La}, \mathrm{Ce}\right) \mathrm{Al}_{2}$ (87) are depressed below the AG value, in agreement with recent calculations by Müller-Hartmann and zittartz (88). This effect has also been observed in the ZnMn system (89), where $T_{K}<T_{C P}$. Boato et al. (90) first pointed out that nonmagnetic resonant states, viewed in the sense of the Friedel-Anderson model, could affect superconductivity. When they added Fe group impurities to $\mathrm{Al}$, for example, they found that $\mathrm{T}_{\mathrm{C}}$ was depressed at a rate intermediate between the strong depression due to magnetic impurities and the weak depression observed for nontransition metal impurities. For Fe group impurities in $\mathrm{Al}$, the resonant state widths are so large 
$(\Delta \gtrsim 1 \mathrm{ev})$ that a local moment cannot exist. However, virtual bound states present at the impurity sites increase the density of states of electrons near each impurity, and the resultant resonance scattering can depress $\mathrm{T}_{\mathrm{C}}$ below the value expected for a simple nonmagnetic impurity.

The first theoretical treatment of the effect of nonmagnetic impurity resonant states on superconductivity was presented by zuckermann (91). He found the initial linear decrease of $\mathrm{T}_{\mathrm{C}}$ to be inversely proportional to $\Delta$. In order to explain the experimental results, however, Zuckermann had to assume much too small widths for the resonant states. Ratto and Blandin (92) and Takanaka and Takano (93) extended zuckermann's theory to include the coulomb repulsion between d electrons of opposite spin on the impurity site. The calculation of Ratto and Blandin (RB) for the initial decrease in $T_{C}$ gave fair agreement with the experimental results obtained for dilute Al-Fe group alloys by Aoki and Ohtsuka (94), the largest discrepancy between theory and experiment occurring for AlMn. Using the RB Hamiltonian, Kiwi. and Zuckermann (95) calculated various thermodynamic properties for superconducting alloys, and they suggested that beyond a critical impurity concentration, gapless superconductivity might be observed. The latter was a surprising result in view of the fact that the RB Hamiltonian is timereversal invariant and pair-weakening, in contrast to the pair-breaking result for paramagnetic impurities. 
A more recent theoretical development concerning the effect of nonmagnetic localized states in superconducting alloys is due to Kaiser (96). Starting with the RB Hamiltonian, Kaiser carefully calculated the excitation spectrum and $T_{C}(n)$ for an isotropic BCS superconducting host. He found that Kiwi and Zuckermann's prediction of gaplessness was incorrect due to an error in their calculation of the order parameter, and showed that when the calculation was done correctly there exists no region of gapless superconductivity. According to this theory the alloys remain BCS like with

$$
T_{C}=1.14 \theta_{D} \exp (-1 / f \lambda)
$$

Here $\lambda=N(0) V^{\prime}$ is the coupling constant of the pure BCS host and, for an impurity with angular momentum $l$, the dilution factor $f$ is given by

$$
f=\frac{1-n c d}{1+n c} \text {, }
$$

where

$$
c=\frac{(2 \ell+1) \mathrm{N}^{(0)}}{\mathrm{N}(0)}
$$

and

$$
\mathrm{a}=\frac{\mathrm{N}^{(0)} \mathrm{v}_{\mathrm{c}}^{\mathrm{eff}}}{\lambda}
$$


$\mathrm{N}_{\ell}(0)$ is given by Equation 31. The denominator in $f$ is a dilution effect due to the participation of the l-orbital electrons in superconductivity via the resonance scattering. The numerator is a pair-weakening effect due to the coulomb repulsion between opposite spin l-orbital electrons. The $v_{C}^{e f f}$ term in $d$ is the effective coulomb potential proposed by Schrieffer and Mattis (97),

$$
v_{c}^{\text {eff }}=\frac{v_{c}}{1+\left(v_{c} / \pi E_{l}\right) \tan ^{-1}\left(E_{l} / \Delta\right)}
$$

which arises from correlations whereby a spin-up electron hops onto an impurity site as a spin-down electron hops off, and vice versa.

The resultant dependence of $T_{C}(n)$ is anomalously steep in its initial descent and approximately exponential:

$$
T_{C}=T_{C p} \exp [-A n /(1-D n)],
$$

where

$$
A=c(1+d) / \lambda
$$

and

$$
D=c d
$$

A critical concentration for the complete suppression of superconductivity 


$$
n_{c r}=\frac{N(0)(2 \ell+1) \lambda}{N_{l}(0)^{2} v_{c}^{e f f}}
$$

is also predicted by the Kaiser model.

The system which best seems to satisfy the conditions of the Kaiser theory is Thce (98). Figure 6 shows the reduced transition temperature of ThCe alloys as a function of Ce concentration at various pressures compared with Kaiser's theory. In addition, systems exhibiting Isf behavior, such as AlMn, for example, are described by the modified exponential $T_{C}(n)$ proposed by Kaiser. Huber and Maple (99) have proposed that this behavior may be a general result for the superconducting-normal phase boundary when the characteristic spin fluctuation temperature $T_{0} \gg T_{C p}$

Morandi (100) and Riblet (101) have presented models to describe the effect of lsf on the superconductivity of Ir based alloys. Morandi used the exchange enhanced model of Lederer and Mills (102) and found an expression for $T_{C}(n)$ considerably more complicated than Kaiser's but which gave qualitative agreement with experiment. Riblet fitted his experimental $T_{C}(n)$ curves for IrNi, IrCo, and IrFe with the McMillan (103) expression modified by the addition of a paramagnon coupling constant proportional to the impurity concentration.

Bennemann (104) has taken a completely different 
THIS PAGE

WAS INTENTIONALLY

LEFT BLANK 
Figure 6. Reduced transition temperature of ThCe alloys versus Ce concentration at various pressures from Huber and Maple (98). The solid lines represent the result of Kaiser's theory (Equation 48) fitted to the data by the method of least squares. 


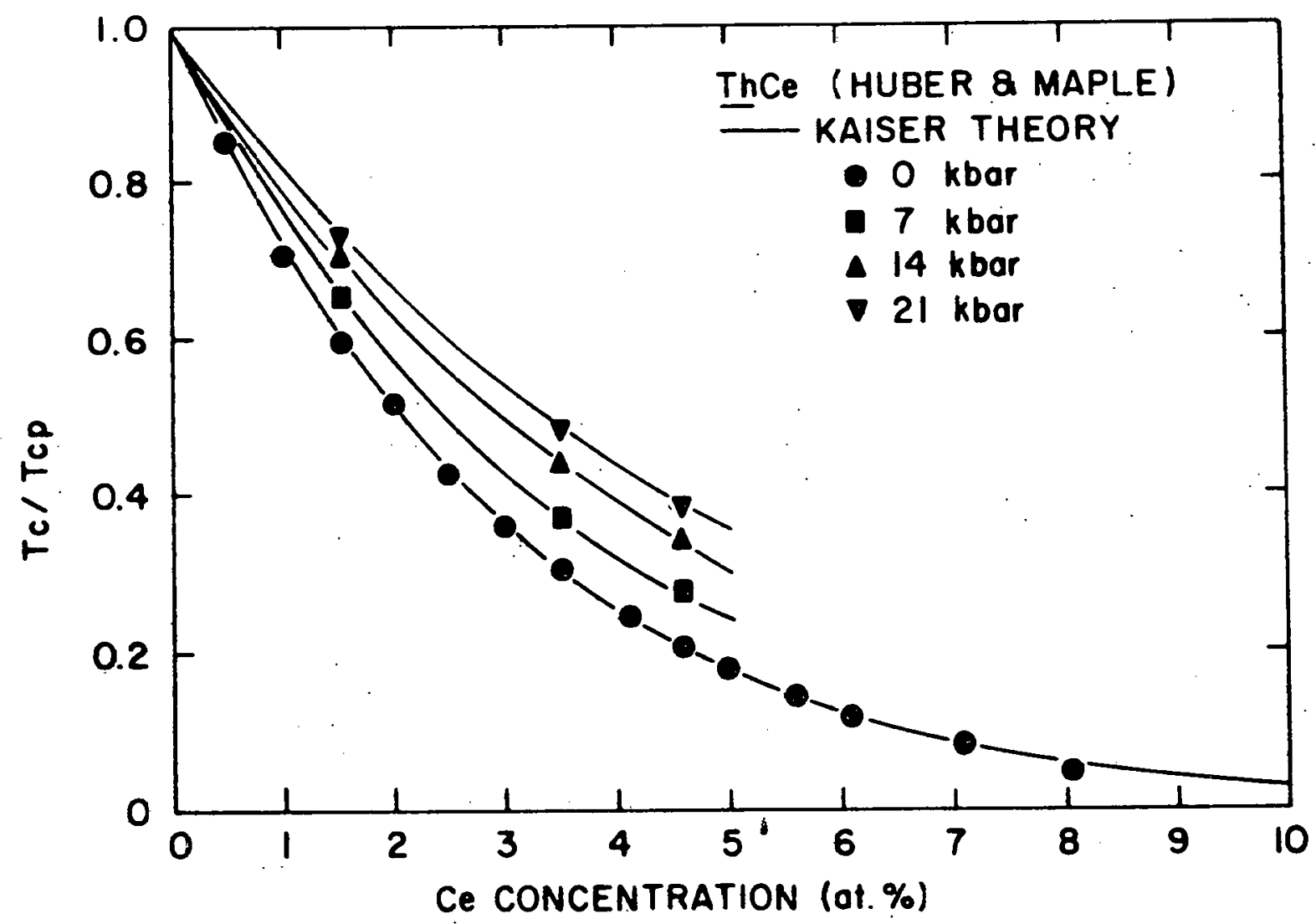

品 
approach to this problem. He has assumed that localized spin fluctuations break pairs and obtained a $T_{c}(n)$ curve of the same general shape as the experimental $T_{C}(n)$ curves for AlMn and AlCr by assuming a pair-breaking parameter $\alpha$ which varied at $T^{2}$. However, when the temperature dependence of $\alpha / \alpha_{c r}$ for 1 in is calculated directly from Equation 40 and experiment, it is found to vary as $\left[a+b(T / T C p)^{1 / 2}\right]$ (46, 47). Also, the critical field curves of AlMn (105) and the specific heat jumps at $\mathrm{T}_{C}$ of AlMn (106) follow the BCS law of corresponding states, indicating that pair-breaking interactions are not present.

Rivier and MacLaughlin (107) have treated the passage from the nonmagnetic to the magnetic regime in superconducting alloys with a lsf model, and hence have bridged the gap between the AG and Kaiser theories. In their model, a single parameter, the characteristic spin fluctuation temperature, $\mathrm{T}_{0}$, characterizes the material and determines the degree to which a local moment picture applies. The AG result for $T_{C}(n)$ is obtained for temperatures $T_{2} T_{0}$ in the limit $\mathrm{T}_{0} \ll \mathrm{T}_{\mathrm{CP}^{\prime}}$ whereas an exponential dependence of $\mathrm{T}_{\mathrm{C}}$ on $\mathrm{n}$, similar to Kaiser's result, is found for $\mathrm{T}_{0} \gg \mathrm{T}_{\mathrm{cp}}$. The effects of lsf in both the weakly magnetic and strongly magnetic regimes have also been studied by zuckermann (108) and Paton and zuckermann (109). 
E. Previous Measurements on ThU

Maple and coworkers (110) have presented strong evidence that the ThU alloy system shows Isf phenomena. A number of normal state properties point to this interpretation. The incremental normal state electrical resistivity, $\Delta \rho$, is described by the expression $\Delta p=\rho_{0}\left[1-\left(\mathrm{T} / \mathrm{T}_{0}\right)^{2}\right]$, where $\mathrm{T}_{0} \sim 100 \mathrm{~K}$. This value of $\mathrm{T}_{0}$ may be compared to $530 \mathrm{~K}$ for AlMn and $1200 \mathrm{~K}$ for $\mathrm{AlCr}$, as determined from their low temperature electrical resistivities (58). The temperature of the resistance minimum, $T_{\text {min }}$, obeys the relation $T_{\min } \propto n^{1 / 3}$, consistent with an incremental resistivity which varies as $\left[1-\left(\mathrm{T} / \mathrm{T}_{0}\right)^{2}\right]$ and a lattice contribution that varies as $\mathrm{T}^{5}$ for pure $T h(111)$. This behavior of $\Delta p$ and $T_{\min }$ for. ThU is quite different from the $\Delta \rho \propto-\ln T$ and $T_{\min } \propto \mathrm{n}^{1 / 5}$ behavior found in Kondo systems $(42,43)$ where the impurities sustain well-defined local moments. Additional evidence for the presence of lsf was provided by the observation of a large peak in the thermoelectric power centered at approximately $70 \mathrm{~K}$. Such "giant" thermoelectric powers have been observed in a number of impurity systems $(42,43,112)$ peaking about their characteristic temperatures, whether they be Kondo or lsf systems. Finally, the effective moment determined from the incremental susceptibility, $\Delta X=\Delta X_{\text {ThU }}-\Delta X_{T_{h^{\prime}}}$ decreased with decreasing temperature below approximately $170 \mathrm{~K}$. In the temperature region from about $170 \mathrm{~K}$ to $300 \mathrm{~K}, \Delta X$ could 
be fitted to a Curie-Weiss law giving an effective moment of $3.43 \pm 0.20 \mu_{B^{\prime}}$ where $\mu_{B}$ is the Bohr magneton. This value of the effective moment is close to that expected for a $U$ 5f-electron configuration of either two $\left(3.58 \mu_{B}\right)$ or three $\left(3.62 \mu_{B}\right)$ f electrons. All the above normal state measurements indicate that the ThU alloy system is described by a lsf model.

Superconductivity measurements of the ThU system give additional support to a lsf interpretation. Maple et al. (110) found an initially large rate of depression of $\mathrm{T}_{\mathrm{C}}$ $(7.95 \mathrm{~K} / \mathrm{at} . \% \mathrm{U})$ with $\mathrm{T}_{\mathrm{C}}(\mathrm{n})$ exhibiting positive curvature for increasing $n$, in sharp contrast to the AG theory prediction for a system with paramagnetic impurities. This $T_{C}(n)$ behavior is quite similar to that observed in the AlMn

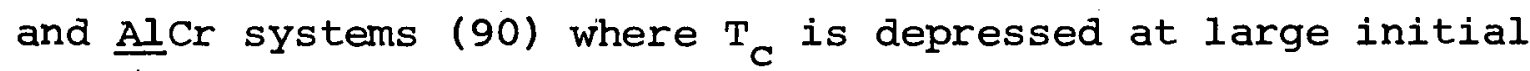
rates of $14 \mathrm{k} / \mathrm{at} . \% \mathrm{Mn}$ and $8.1 \mathrm{~K} / \mathrm{at} . \% \mathrm{Cr}$ and then has a positive curvature at higher concentrations. Luengo et al. (113) have measured the low temperature specific heat of a number of Thu alloys in the normal and superconducting state and found that the jump in specific heat at $\mathrm{T}_{c}$ closely followed the BCS law of corresponding states, thereby indicating that the depression of $T_{C}$ in this alloy system is due to pairweakening, rather than pair-breaking interactions. They also found that the electronic specific heat coefficient was strongly enhanced at a rate $d_{\gamma} / d_{n}=2.7 \mathrm{~mJ} / \mathrm{mole}-\mathrm{K}^{2}$ at. $\% \mathrm{U}$ 
at the lowest $U$ concentrations. If it is assumed that the initial increase in $\gamma$ with $U$ concentration is due to uranium $5 f$ resonant states, the local density of states at the Fermi level (for one spin direction) contributed by each $U$ atom would be 57 states/eV-U atom. Huber and Maple (114) have found that the depression of $\mathrm{T}_{\mathrm{C}}$ for ThU alloys decreases with pressure, and proposed that this result indicates that the lifetime of the lsf decreases with pressure.

The $\mathrm{T}_{C}(n)$ data of Maple et al. (110) have been compared with a number of previously mentioned models. The pairbreaking theory of Müller-Hartmann and zittartz (8I) gives a good fit by assuming $S=1 / 2$ and $T_{K} / T_{C p}=32$ in Equation 42 . However, in this theory, the calculated specific heat jumps at $\mathrm{T}_{\mathrm{C}}$ in the limit $\mathrm{T}_{\mathrm{K}} / \mathrm{T}_{\mathrm{CP}} \gg 1$ tend towards $\mathrm{AG}$ (88), contrary to the specific heat results. Kaiser's theory also gives a good fit $(46,47)$, but the density of states obtained from the depression of $\mathrm{T}_{\mathrm{C}}$ is small and negative, a clearly unphysical result.

Maple et al. (110) found that Morandi's model (100) gave a close fit. They also used Equation 40 and their experimental data to find the temperature dependence of the AG pair-breaking parameter $\alpha$ and found that $\alpha \propto\left(\mathrm{T} / \mathrm{T}_{\mathrm{Cp}}\right)^{1 / 2}$, which is in disagreement with the Bennemann pair-breaking model's prediction of $\alpha \propto T^{2}(104)$. Riblet has also found good agreement with $T_{C}(n)$ for ThU using his model (101). 
F. Objectives

The objective of this study has been to determine the magnetic state of $U$ impurities in Th from their effect on the superconductivity of Th by measuring the critical field curves, $\mathrm{H}_{\mathrm{C}}(\mathrm{T})$, of a number of ThU alloys and comparing the experimental results with the $A G$ and $B C S$ theories. An additional comparison was possible because the specific heat jump at $\mathrm{T}_{\mathbf{C}}$ calculated from the theories could be compared with the value calculated from the slope of the experimental $\mathrm{H}_{\mathrm{C}}(\mathrm{T})$ curve at $\mathrm{T}_{\mathrm{C}}$ using Rutger's formula, Equation 15. 


\section{EXPERIMENTAL APPARATUS AND PROCEDURE}

A. Sample preparation and Analysis

The ThU and pure Th samples were provided by D. T. Peterson's group of this laboratory. The procedure in the ThU preparation was to first prepare a master alloy containing 1.88 at. $\%$ U by arc melting together the appropriate constituents, under an argon atmosphere in a water cooled copper hearth, with a tungsten tip. The master alloy was then separated into small pieces and diluted with pure thorium, by the above method, to attain the desired concentrations. The samples, which weighed approximately $11.5 \mathrm{~g}$, were then swaged to dimensions of approximately 0.1 inches in diameter by 1.5 inches in length, electropolished in a perchloric acid and methanol solution, sealed in a Ta crucible and annealed at $1200^{\circ} \mathrm{C}$ for one week, then slow cooled to room temperature. A chemical analysis was performed on ali the ThU samples by J.S. Fritz's group of this laboratory using a spectrophotometric method which was accurate to the nearest 0.001 at. $\%$ U. The results are given in Table 1 . The solid solubility of $U$ in Th has been reported to be 1.5 at.\% at $800^{\circ} \mathrm{C}(115)$, and so for the low $\mathrm{U}$ concentrations used in this study, we are assured of having good solid solutions (116). 
Table 1. Atomic percent $U$ determined by chemical analysis

\begin{tabular}{llll}
\hline $\begin{array}{c}\text { Nominal } \\
\text { at.\% U }\end{array}$ & Run 1 & Run 2 & $\begin{array}{l}\text { Average } \\
\text { at.\% U }\end{array}$ \\
\hline 0.025 & 0.030 & 0.030 & 0.030 \\
0.050 & 0.051 & 0.053 & 0.052 \\
0.050 & 0.060 & 0.057 & 0.059 \\
0.075 & 0.076 & 0.077 & 0.077 \\
0.100 & 0.101 & 0.102 & 0.102 \\
0.125 & 0.126 & 0.127 & 0.127 \\
0.150 & 0.149 & 0.150 & 0.150 \\
0.150 & 0.161 & 0.161 & 0.161 \\
0.200 & 0.212 & 0.217 & 0.215 \\
\hline
\end{tabular}

B. Cryostat

Temperatures ranging from $1.4 \mathrm{~K}$ to $0.3 \mathrm{~K}$ were attained using the $\mathrm{He}^{3}$ cryostat shown in Figure 7. Temperatures above $1.2 \mathrm{~K}$ were obtained by admitting 5 or $6 \mathrm{~mm}$ of $\mathrm{He}^{3}$ gas to the $\mathrm{He}^{3}$ system to thermally link the $\mathrm{He}^{3}$ and $\mathrm{He}^{4}$ evaporation chambers. Temperatures below $1.2 \mathrm{~K}$ were maintained by pumping on $\mathrm{He}^{3}$ which had been condensed in the $\mathrm{He}^{3}$ evaporation chamber.

A copper holder for three symmetrically spaced coil forms and the germanium resistance thermometer, GR-928, was bolted onto the bottom of the $\mathrm{He}^{3}$ evaporation chamber. The surfaces in this and other pressure contacts were coated with Apiezon $\mathrm{N}$ grease in order to improve thermal conductivity at the contact boundary. Heat leaks into the system via 


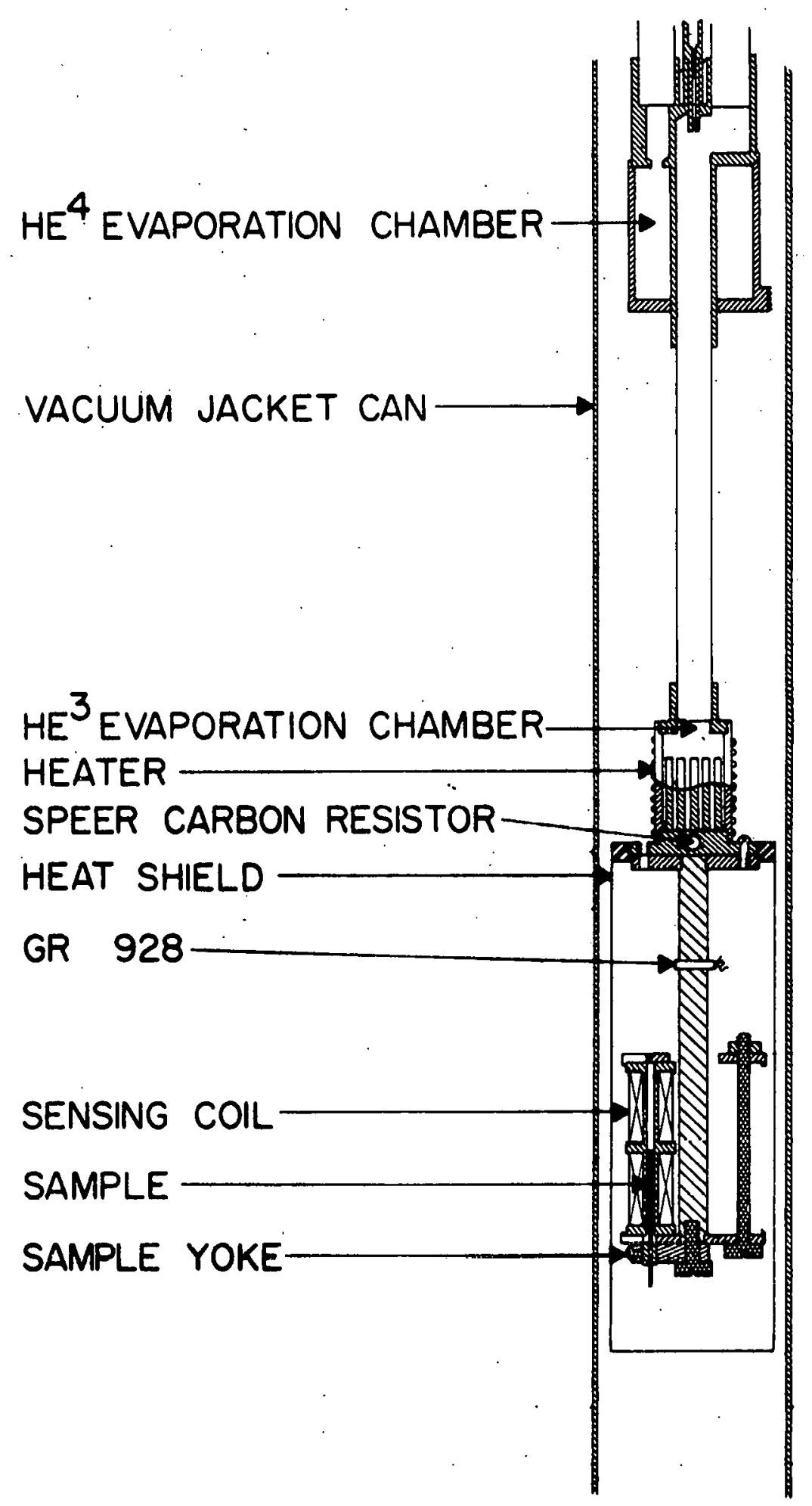

Figure 7. Cross-section of $\mathrm{He}^{3}$ cryostat. 
electrical connections were minimized by cementing the leads from the germanium resistance thermometer and transition sensing coils to the coil holder, $\mathrm{He}^{3}$ evaporation chamber, and $\mathrm{He}^{4}$ evaporation chamber with stycast $2850 \mathrm{GT}$, and radiation effects were minimized by enclosing the coil holder with a copper heat shield connected to the $\mathrm{He}^{3}$ evaporation chamber. The coils, which were used to sense the superconducting transition, were wound on linen fiber coil forms 1.8 inches long with 0.5 inch outer diameters and 0.125 inch bores. In order to make ac susceptibility measurements, each coil was wound with about 1500 turns of No. 40 copper wire on the primary, while each half of the astatic secondary contained 5000 turns of No. 40 copper wire. The turns were cemented to the coil forms with GE 7031 adhesive.

One end of the superconducting samples was coated with Apiezon $\mathrm{N}$ grease and placed in a 0.125 inch collar. Sufficient sample extended above the collar to fill the lower half of the transition sensing coil when the collar was held in the yoke attached to the bottom of the sample holder.

The vacuum jacket can was soldered to the can top by Wood's metal and this seal could easily be broken to change samples. The can top also contained an electrical feedthrough constructed with nylon and stycast 2850 GT to allow the electrical leads to pass from the $\mathrm{He}^{4}$ bath to the vacuum space. 
C. Thermometry and Temperature control

Temperatures were measured using a Honeywell germanium resistance thermometer, GR-928. This thermometer had previously been calibrated by W. R. Decker (117) against the susceptibility of cerium-magnesium-nitrate (CMN) at 58 points from $0.3 \mathrm{~K}$ to $1.3 \mathrm{~K}$, and had been calibrated against the T-58 $\mathrm{He}^{4}$ vapor pressure tables (118) at 12 points. by $W$. R. Decker (117) and at 49 points by L. J. Williams (119) from $1.3 \mathrm{~K}$ to $4.2 \mathrm{~K}$ to form a complete set of data from $0.3 \mathrm{~K}$ to $4.2 \mathrm{~K}$. Figure 8 is a block diagram of the direct current fourlead potentiometric method used to measure the resistance of the germanium resistance thermometer. The resistance was obtained by comparing the voltage drop across the unknown resistance (GR-928) with the voltage drop across the General Radio Type 500-H $1 \mathrm{k} \Omega$ standard resistor for a fixed current. The germanium resistance thermometer constant current power supply is shown in Figure 9. Other components of the system included a Guideline Type 9180-B potentiometer, a constant current potentiometer power supply, an Eppley standard cell (No. 788370), and a Keithley 150B null detector. Circuit ground was at the low side of the null detector. The constant current potentiometer power supply (120) employed an Analog Devices Model $184 \mathrm{~J}$ operational amplifier. A temperature compensated zener diode was used as an input reference voltage. The output current drifted about 1 ppm per day. 


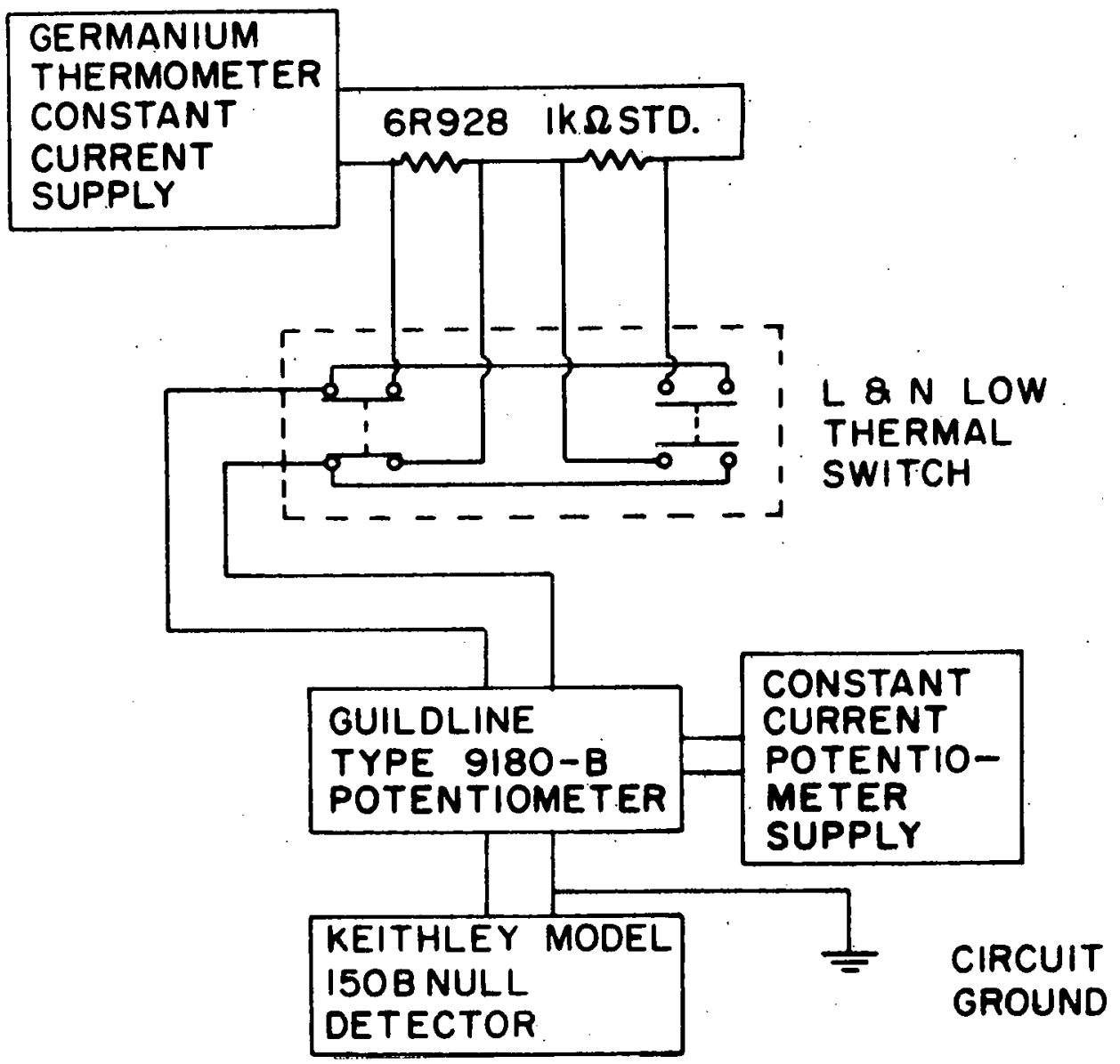

Figure 8. Germanium resistance thermometer circuit. 


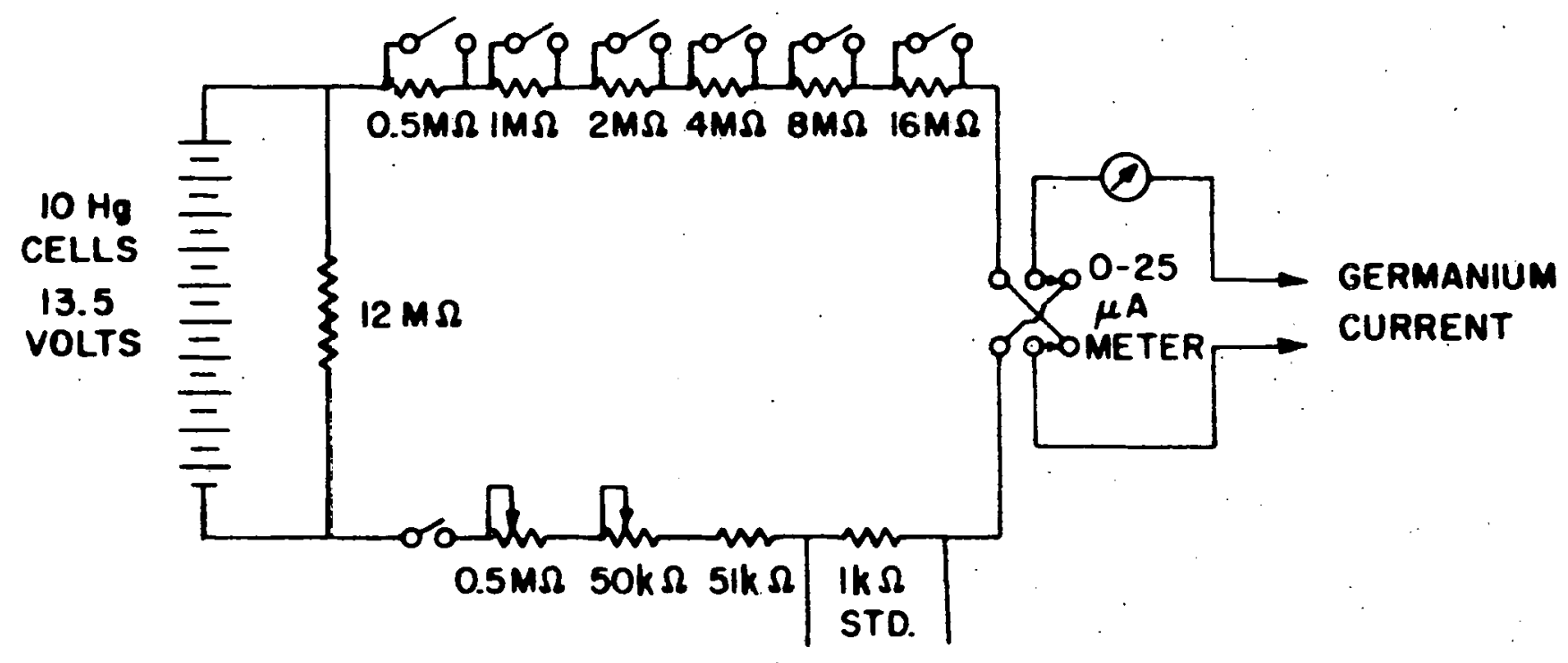

Figure 9. Germanium resistance thermometer constant current power supply. 
The potentiometer was standardized by adjusting the output current of the power supply.

The temperature control circuit is shown in Figure 10. The temperature control circuit used a three-lead direct current. Wheatstone bridge which compared the resistance of a Speer carbon resistor ( $1 / 2 \mathrm{~W}$, nominal $470 \Omega$, grade 1002) mounted on the $\mathrm{He}^{3}$ evaporation chamber with a variable decade resistance (General Radio Type 1434-X decade resistor). The off-balance bridge voltage was amplified by a Keithley Model 150AR null detector and sent to a transistor controlled heater power supply which turned the heater current on (off) whenever the speer carbon resistor's resistance became greater (less) than that of the decade resistor. The heater consisted of about $100 \Omega$ of No. 36 manganin wire wound astatically and cemented to the $\mathrm{He}^{3}$ evaporation chamber with Stycast $2850 \mathrm{GT}$. The temperature could be controlled with Speer resistance variations of less than $0.1 \Omega$, the minimum resistance of the decade resistor. Typically, temperatures were maintained within a precision of $0.0001 \mathrm{~K}$ for the $1 / 2$ hour periods during which a data point was measured.

\section{Magnetic Field Sources}

The applied field at the samples was generated by a Iiquid nitrogen cooled solenoid with an inner diameter of four inches and length of ten inches. The solenoid was 


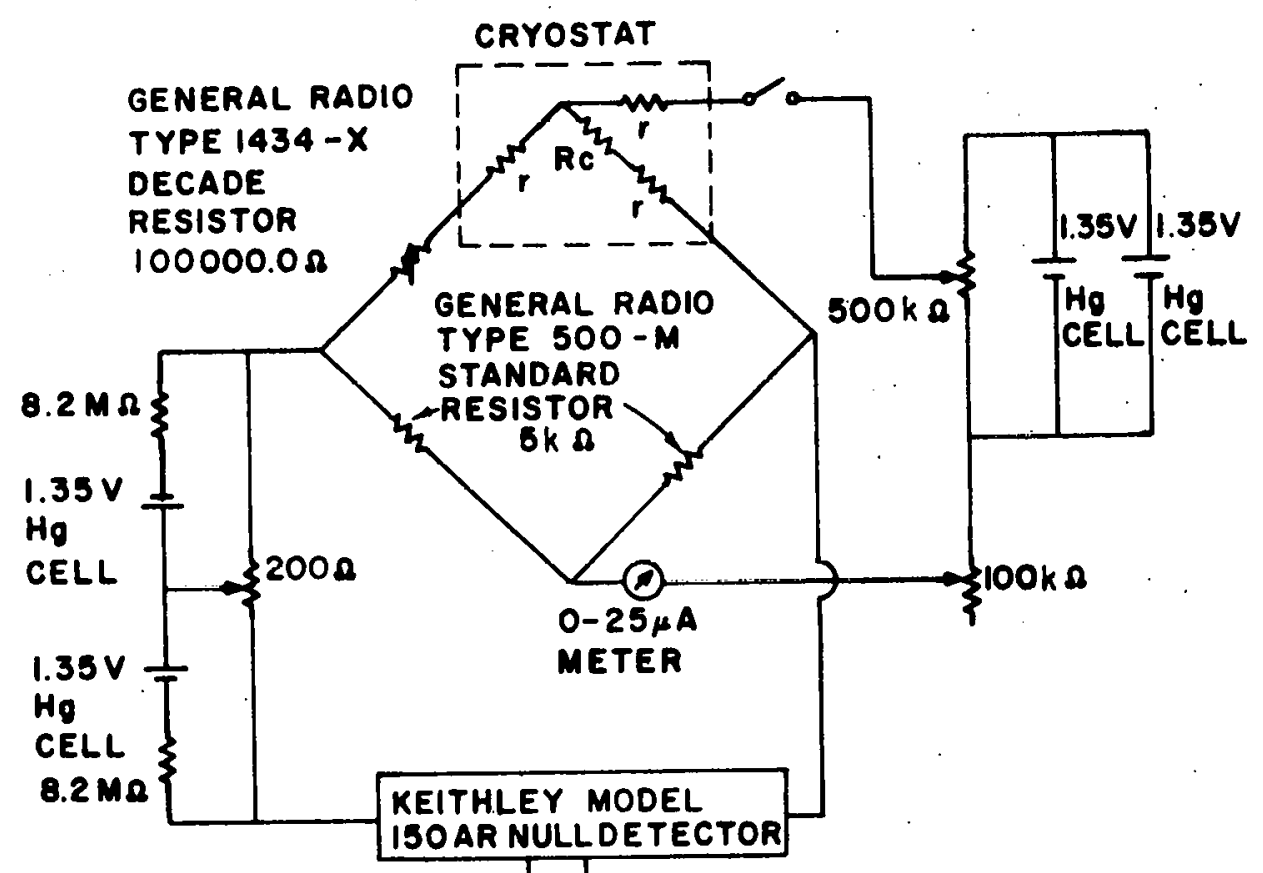

Figure 10. Wheatstone bridge used for temperature control. 
wound with No. 14 square polythermalize coated copper wire with 1138 turns in eight layers and 128 turns overlaid on the ends to compensate for end effects. The layers were separated by 0.031 inch thick teflon strips placed parallel to the solenoid's axis to allow penetration of liquid nitrogen through the windings. A magnetic field homogeneity measurement using a Bell 240 Incremental Gaussmeter showed axial field homogeneity within $0.11 \%$ over a range of $3.81 \mathrm{~cm}$ and within $0.24 \%$ over a range of $5.72 \mathrm{~cm}$ from the center of the solenoid. The solenold was calibrated at liquid nitrogen temperatures by nuclear magnetic resonance in water. Data points were taken at the center of the solenoid for six fields between $1260 \mathrm{Oe}$ and $1360 \mathrm{Oe}$ and yielded a value of $56.599 \mathrm{Oe} / \mathrm{A}$. The solenoid was powered by a Kepco Model KS 36-30M DC power supply with current regulation stable to one part in $10^{4}$. The output current of the power supply was programmed with external resistance provided by a Dekabox DB62 decade resistor and a General Radio Type 1432-N decade resistor connected in series. An increase in the external resistance by $0.1 \Omega$ increased the field by approximately 0.060 0e. The resistance of the solenoid was measured by the direct current four-lead potentiometric circuit shown in Figure 11. Components of the circuit included a Leeds and Northrup $\mathrm{K}-3$ potentiometer, a Dynage BSTC-2C constant voltage supply, an Eppley standard cell (No. 775042), a Leeds and Northrup 


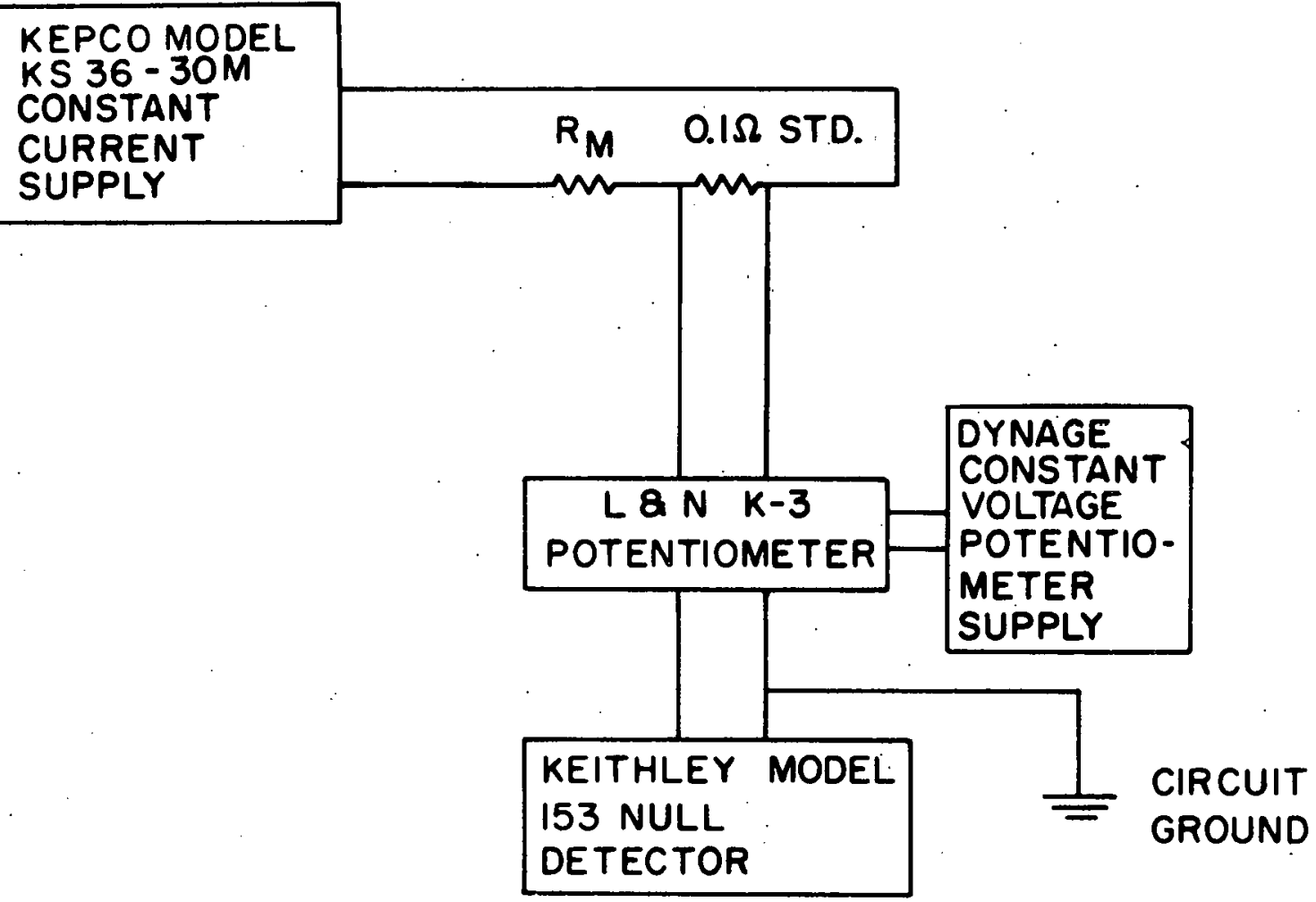

Figure 11. Magnetic field measurement circuit. 
$0.1 \Omega, 15 \mathrm{~A}$ shunt, and a Keithley 153 null detector. The earth's magnetic field was compensated to approximately 0.01 oe with a Helmholtz pair. The current and position required by the Helmholtz pair to cancel the horizontal and vertical components of the earth's magnetic field in the sample region of the cryostat were determined using the Beil 240 Incremental Gaussmeter. The Helmholtz pair power supply is shown in Figure 12 .

\section{E. Mutual Inductance Bridge}

The superconducting transition temperatures of the samples were initially determined using the modified Hartshorn mutual inductance bridge shown in Figure 13. Similar bridges have been described by others (121-124).

The $32.05 \mathrm{~Hz}$ oscillator with a quartz crystal frequency control arove the primary circuit via a Gertsch Model CRTI2AT coaxial ratio transformer. The output of the secondary of the fixed reference mutual inductance lump provided a constant voltage to the Gertsch Model 1011 AC ratio transformer. Proper adjustment of the ratio transformer balanced the inductive component of the signal from the secondary of the sample measuring coils. Quadrature phase signals due to eddy current losses and nonideal inductors in the circuit were nulled out by the resistive network. The voltage introduced into the detection circuit was proportional to 


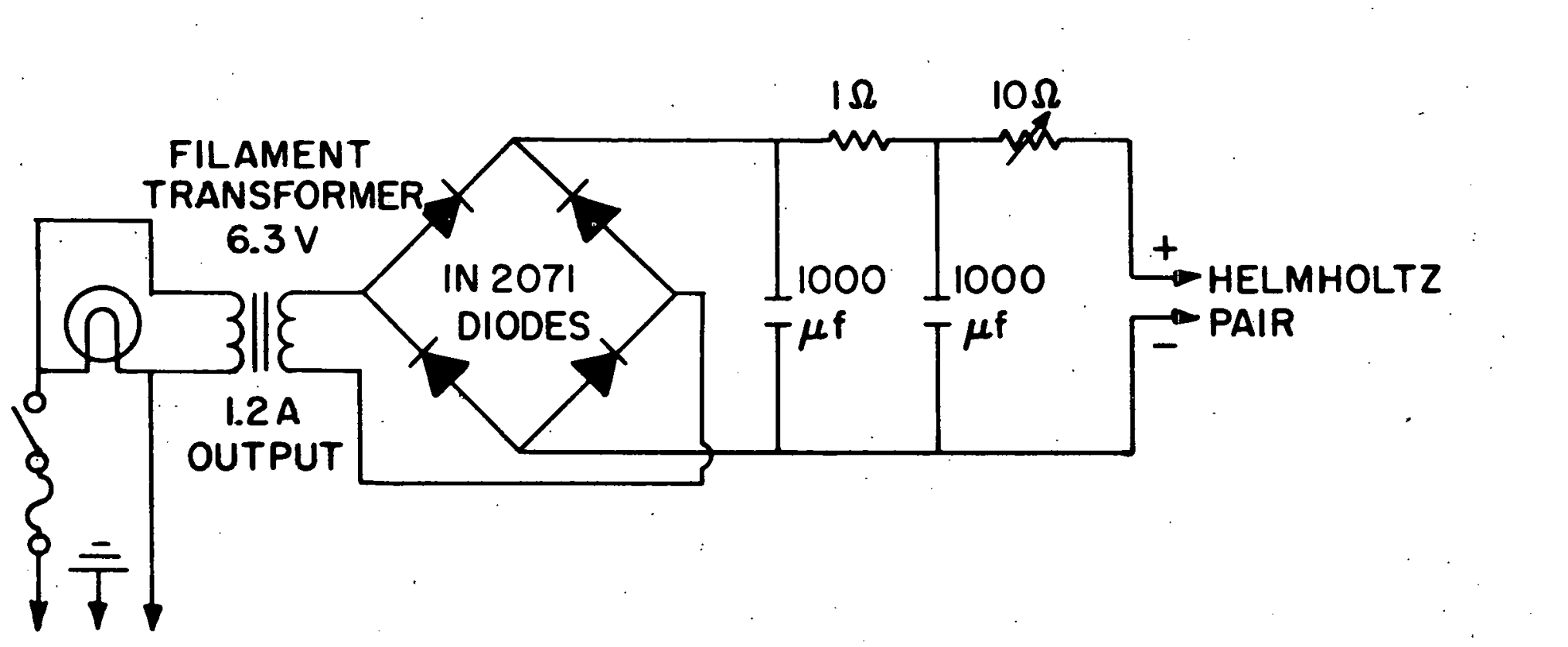




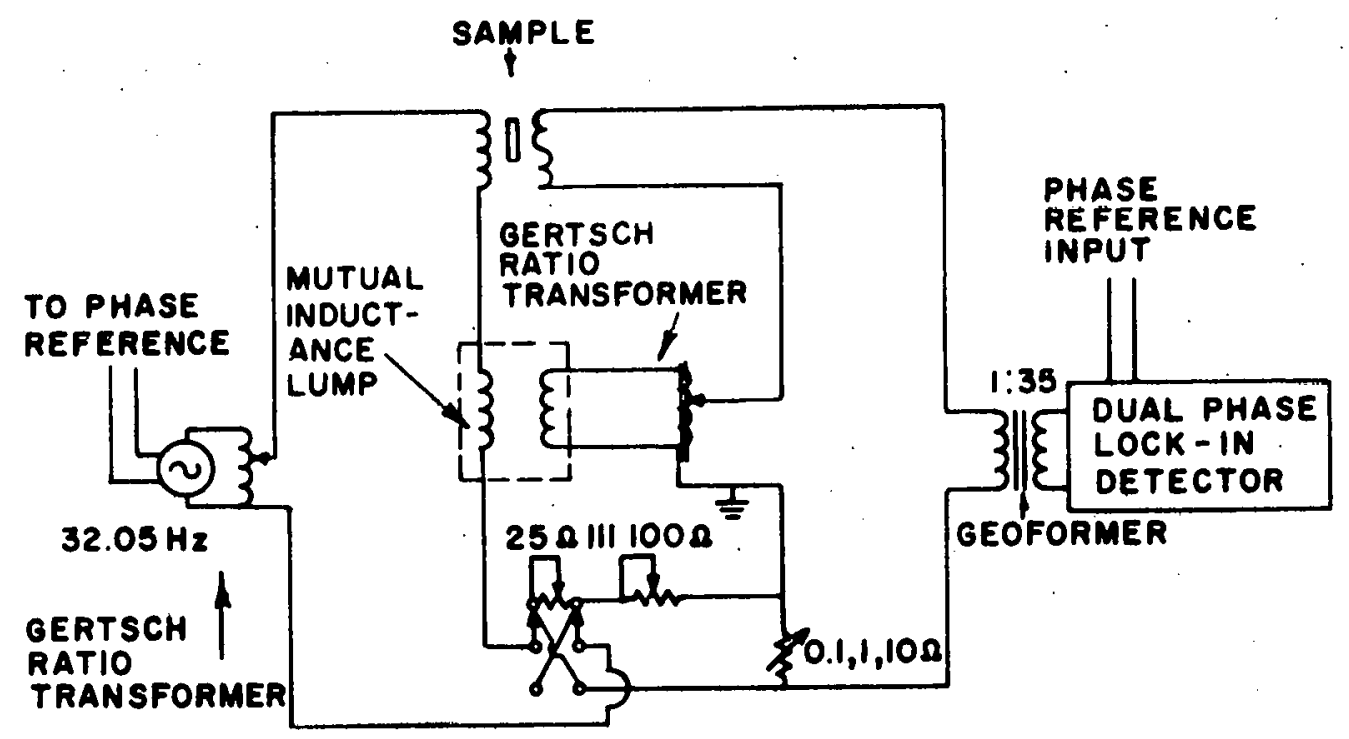

Figure 13. $32.05 \mathrm{~Hz}$ mutual inductance bridge. 
$1 /(111100 \ldots R)$ where $R$ is the setting of the variable resistors. A locally built dual phase lock-in detector (i25; 126) coupled to the circuit through a $35: 1$ Geoformer acted as a null detector.

When. the superconducting sample undergoes a transition from the normal to the superconducting state, it becomes diamagnetic and thus changes the mutual inductance between the primary and secondary of the coils surrounding the sample. This, in turn, varies the reference voltages necessary for a null condition in the secondary circuit.

\section{F. Critical Field Measurements}

The superconducting transitions in the applied magnetic fields were determined isothermally by a ballistic induction technique first reported by cochran et al. (127). The sample, which filled the lower half of the astatically wound transition sensing coil, was placed in the cryostat at the center of the liquid nitrogen cooled solenoid with the magnetlc field slightly less than $\mathrm{H}_{\mathrm{C}}$. The applied magnetic field was increased stepwise through $\mathrm{H}_{\mathrm{C}}$ and changes in flux linking the sample were measured by a ballistic galvanometer in series with the transition sensing coil. The average. permeability of the specimen as it made a superconducting to normal transition was determined from the galvanometer deflection accompanying each step, since each deflection 
was proportional to the flux which enters the sample. The average permeability of the sample at the $\mathrm{m}^{\text {th }}$ step is defined as

$$
\mu_{m}=\sum_{i=1}^{m} \delta_{i}^{\prime} \sum_{\text {all }}{ }_{\text {steps }} \delta_{i}
$$

where $\delta_{i}$ is the deflection accompanying the $i^{\text {th }}$ step. $H_{c}$ was then determined by extrapolating a plot of $\mu$ versus the applied field to $\mu=1$ for the temperature maintained. The procedure followed in taking data was to stabilize the temperature, wait about 15 minutes, then measure the superconducting to normal and normal to superconducting transitions. 


\section{RESULTS AND DISCUSSION}

Critical field curve data which were obtained for seven different ThU alloys are listed in Appendix A and shown graphically in Figures 14 and 15. The data show excellent agreement with the BCS critical field curves indicated by the solid curves, but marked disagreement with the AG theory critical field curves, as illustrated for Th-0.127 at.\% U by the dashed curve. The procedure used to obtain the theoretical curves will be discussed in detail later.

The primary factor limiting the accuracy of these results is the presence of hysteresis in the superconducting transitions. For our purposes we define hysteresis as the magnitude of the difference between the values of $\mathrm{H}_{\mathrm{C}}$ obtained in increasing and decreasing magnetic fields for a given transition. The effect of annealing on the magnitude of the hysteresis is illustrated in Figure 16 by the superconducting transition obtained for Th-0.059 at.\% U near $\mathrm{T}=0.350 \mathrm{~K}$. The transition after the sample had been annealed at $800^{\circ} \mathrm{C}$ for one-half hour is indicated by the open circles and the magnitude of the hysteresis is approximateiy $6 \% \mathrm{H}_{\mathrm{C}}$. Annealing at temperatures close to the alloy phase transition tended to homogenize the samples and, in addition to reducing the hysteresis considerably, the transitions were generally sharpened. As shown in Figure 16, the hysteresis loop after the anneal tended to center on the previously 


\section{THIS PAGE \\ WAS INTENTIONALLY \\ LEFT BLANK}


Figure 14. Critical field data for the ThU alloys exhibiting small hysteresis plotted versus temperature. The solid curves indicate the BCS theory critical field curves and the dashed curve indicates the AG theory critical field curve for Th-0.127 at. $\% \mathrm{U}$. The bars on the Th-0.030 at. $\% \mathrm{U}$ and the Th- 0.059 at. $\% \mathrm{U}$ data show the width of the superconducting transitions. 
64

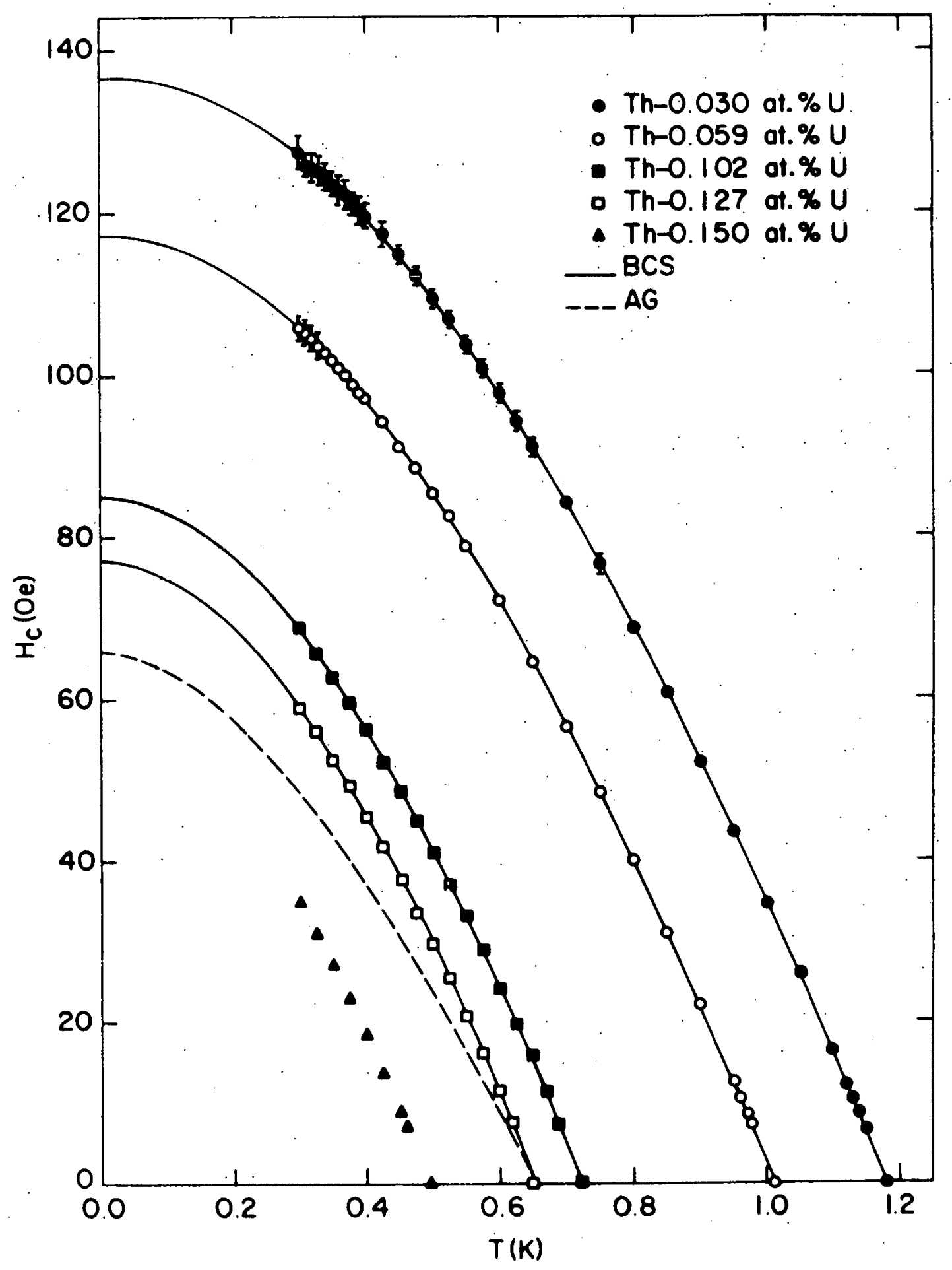


THIS PAGE

\section{WAS INTENTIONALLY \\ LEFT BLANK}


Figure 15. Critical field data for the ThU alloys exhibiting large hysteresis plotted versus temperature. The solid curves indicate the BCS critical: field curves. The bars on the data show the width of the superconducting transitions. 
66

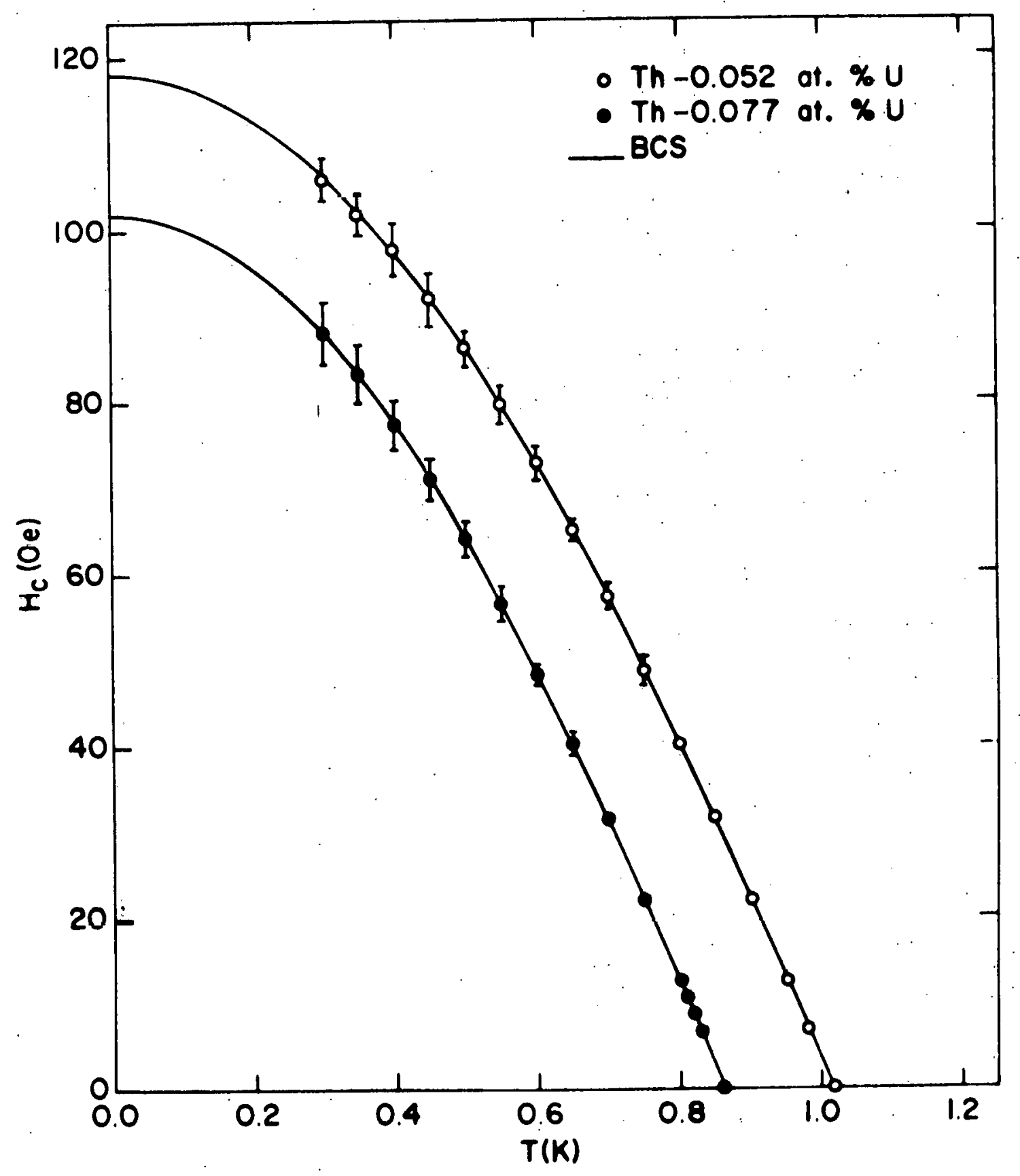




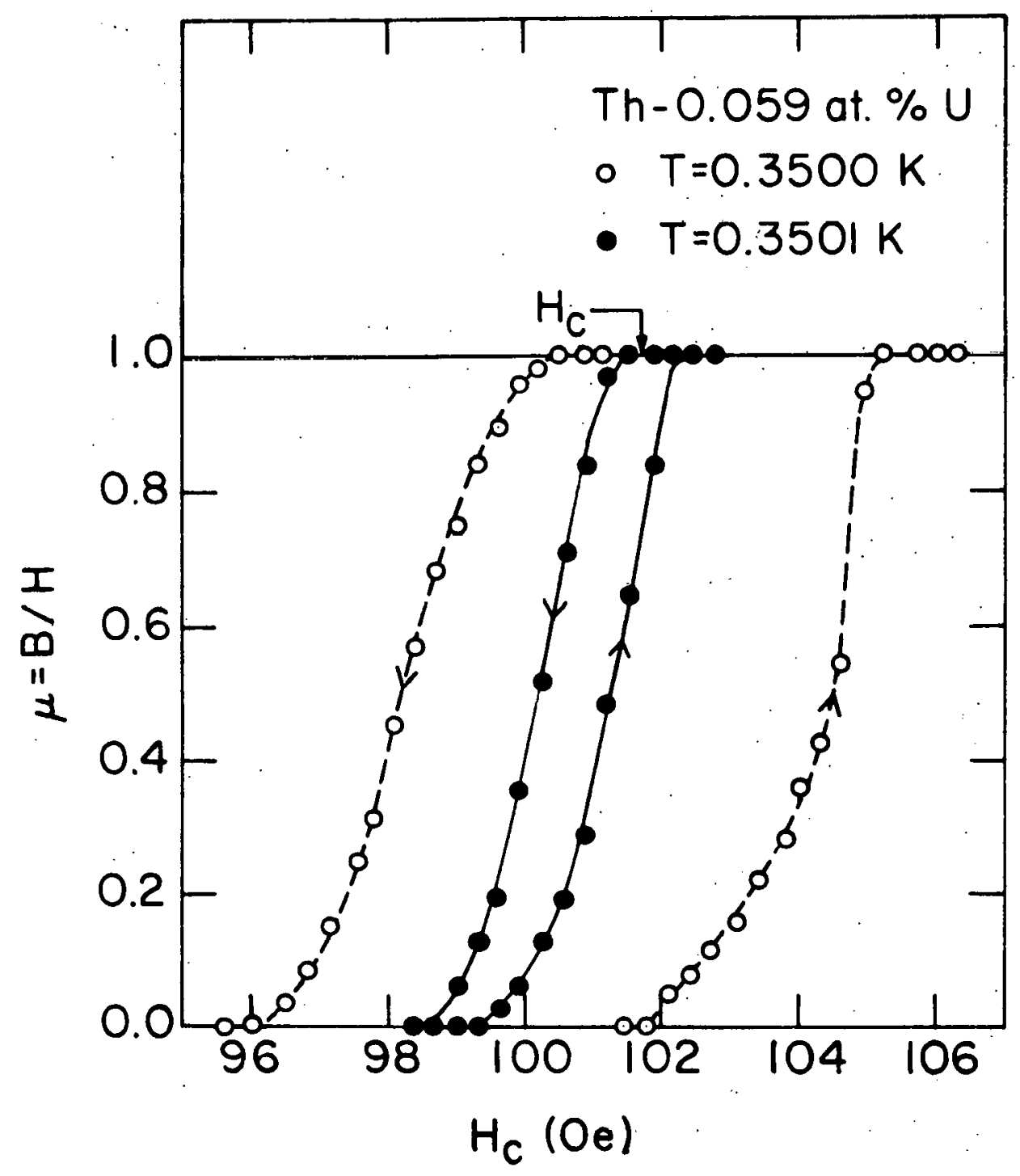

Figure 16. Superconducting transitions for Th-0.059 at.\% U annealed at $8000^{\circ} \mathrm{C}$ for one-half hour (open circles) and at $12000^{\circ}$ for one week (dark circles). $\mathrm{H}_{\mathrm{C}}$ is an average critical field for the transition with the least hysteresis. 
obtained larger hysteresis loop. Because of this behavior, the value of $\mathrm{H}_{\mathrm{C}}$ for the smaller hysteresis loop was chosen as the midpoint of the values of $\mathrm{H}_{\mathrm{C}}$ obtained in increasing and decreasing magnetic fields.

Generally speaking, the magnitude of the hysteresis found for the annealed samples was about a $1 \%$ effect: The Th-0.059, -0.102, -0.127, and -0.150 at.\% U samples each had a maximum hysteresis of about $1 \% \mathrm{H}_{\mathrm{C}^{\prime}}$ but there were deviations from this pattern. For example, the Th-0.030 at.\% $\mathrm{U}$ sample had a maximum hysteresis of about $3 \% \mathrm{H}_{\mathrm{C}}$ at lower temperatures; and for two samples, Th-0.052 at.\% $\mathrm{U}$ and Th-0.077 at.\% $U$, the superconducting transitions observed at low temperatures were very broad and the maximum hysteresis was as large as $7 \% \mathrm{H}_{\mathrm{C}}$. For some reason, the $1200^{\circ} \mathrm{C}$ anneal did not improve the quality of these samples. Hence, the low temperature values of $\mathrm{H}_{\mathrm{C}}$ obtained for these two samples are much less accurate than the low temperature values of $\mathrm{H}_{\mathrm{C}}$ obtained for the other samples and probably should be ignored. The magnitudes of the hysteresis obtained for the superconducting transitions of the alloys are listed in Appendix A.

One additional feature of the superconducting transitions obtained for the ThU alloys is illustrated in Figure 16. Each of the alloys displayed the same total galvanometer deflections if one stepped through a given 
superconducting transition several times in increasing and decreasing magnetic fielads, indicating that the alloys exhibited the Meissner effect.

The superconducting transition temperatures for the above alloys were determined by least squares fitting the $\mathrm{H}_{\mathrm{C}}(\mathrm{T})$ data near $\mathrm{T}=\mathrm{T}_{\mathrm{C}}$ to $\mathrm{H}_{\mathrm{C}}(\mathrm{T})=\mathrm{A}+\mathrm{BT}^{2}$. and extrapolating to $\mathrm{H}_{\mathrm{C}}=0$. In addition, the transition temperatures for the nominally pure Th sample, $T_{\mathrm{CP}^{\prime}}$, and for the Th-0.161 at.\% U and $\mathrm{Th}-0.215$ at.\% $U$ samples were determined from a careful ac susceptibility measurement. The $T_{c}(n)$ datia are presented in Figure 17 where the reduced transition temperature, $\mathrm{T}_{\mathrm{C}} / \mathrm{T}_{\mathrm{Cp}}$ ' for the alloys are plotted versus uranium concentration. The solid line in the figure represents the result of the AG theory (Equation 40) with $T_{C P}=1.360 \mathrm{~K}$ and $\mathrm{n}_{\mathrm{cr}}=0.157$ at.\% $\mathrm{U}$. The value of $\mathrm{n}_{\mathrm{cr}}$ was obtained by using Equation 41 to fit the lower uranium concentration alloys. These $T_{C}(n)$ data are in good agreement with those obtained by Maple et al. (110). The depression of $\mathrm{T}_{\mathrm{c}}$ is initially linear with increasing $U$ concentration and then exhibits positive curvature for larger concentrations, in sharp disagreement with the result of the AG theory (64) for a superconductor containing paramagnetic impurities but in good agreement with the Kaiser theory (96).

The next step in the analysis of the $H_{C}(T)$ data was to compare the $H_{C}(T)$ data with the predictions of the $A G$ and 
THIS PAGE

\section{WAS INTENTIONALLY \\ LEFT BLANK}


Figure 17. Reduced transition temperature, $\left(T_{c} / T_{C p}\right)$, of ThU versus $U$ concentration. The solid curve represents the result of the $A G$ theory (Equation 40) with $\mathrm{T}_{\mathrm{CP}}=1.360 \mathrm{~K}$ and $\mathrm{n}_{\mathrm{Cr}}=0.157$ at. $\% \mathrm{U}$. The bars on the one point indicate the uncertainty in the uranium concentration for that sample (Th-0.215 at.\% U). 


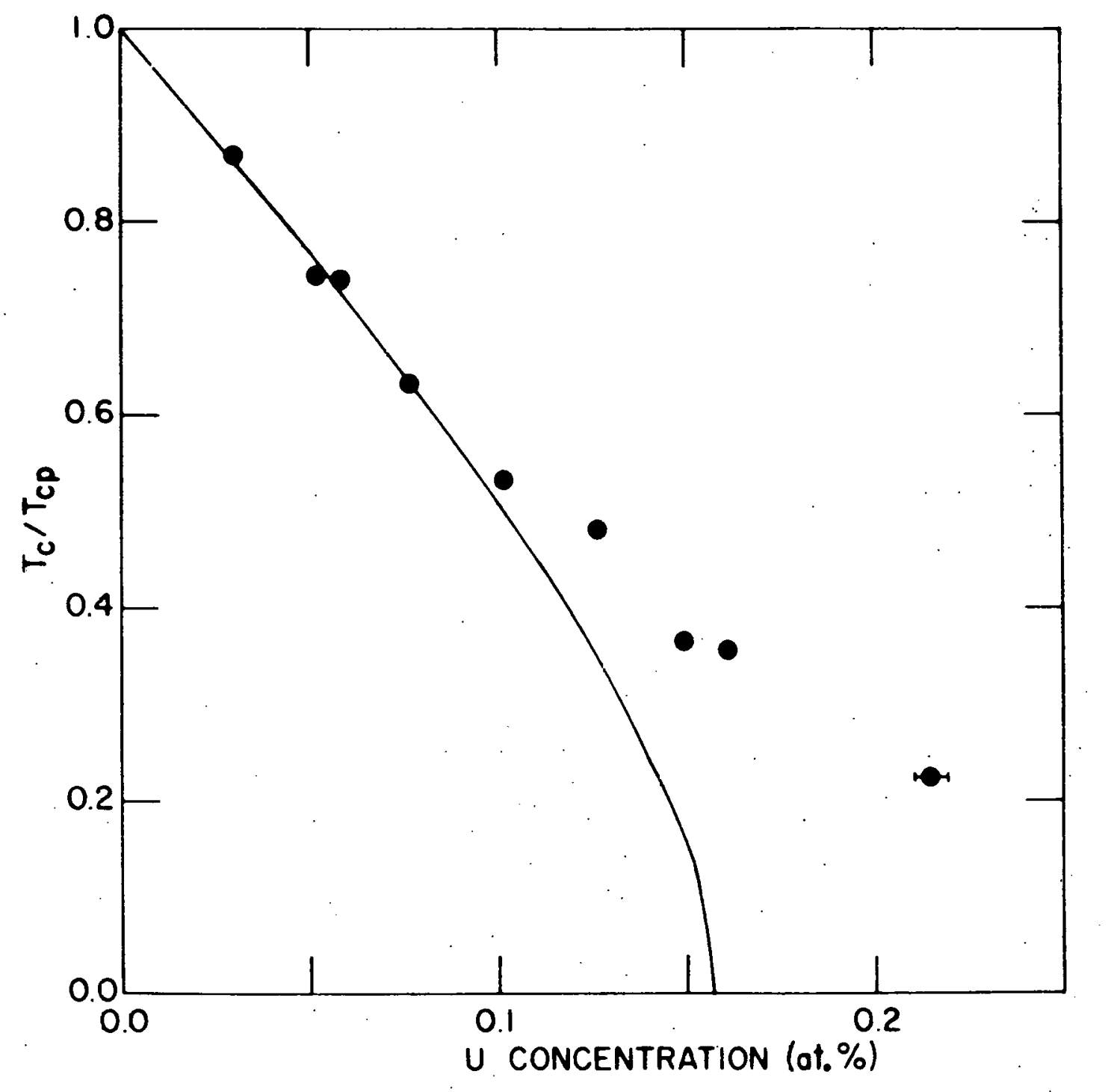


the BCS theories. The ratios of $\mathrm{T}_{C} / \mathrm{T}_{\mathrm{CP}}$ for each alloy for which $\mathrm{H}_{\mathrm{C}}(\mathrm{T})$ data were obtained were used to calculate the AG theory critical field curves (66) using the method explained in detail by Decker (117). When these calculations were completed, it was found that the $A G$ theory $H_{C}(T)$ values were considerably smaller than the $\mathrm{H}_{C}(\mathrm{~T})$ data, as shown in detail in Figure 14 where the AG theory critical field curve for Th-0.127 at.\% $U$ is indicated by the dashed curve. Indeed, the $\mathrm{H}_{\mathrm{C}}(\mathrm{T})$ data lay much closer to the BCS theory critical field curves.

In order to compare the data with the BCS theory in greater detail, it was necessary to determine a value of $\mathrm{H}_{\mathrm{O}}$ for each alloy. The usual procedure used for obtaining $\mathrm{H}_{0}$ is to fit experimental $\mathrm{H}_{\mathrm{C}}(\mathrm{T})$ data to Equation 17 for $\mathrm{T}<0.25 \mathrm{~T}_{\mathrm{C}^{\prime}}$ thereby determining both $\mathrm{H}_{0}$ and $Y$ for a given sample, but this method was inapplicable here because the $\mathrm{He}^{3}$ evaporation refrigerator used in this experiment was unable to attain sufficiently low temperatures. Hence, instead of using Equation 17 to determine $\mathrm{H}_{0}$ and $\varphi$, we have used two different methods. First, we fitted the data to the simple BCS theory to verify that the theory applied rather well, and then we used a more complicated anisotropy corrected BCS expression (40) for the final fitting.

For the analysis where simple BCS was used, values of $\mathrm{H}_{0}$ were obtained for all values of $t^{2}=\left(T / T_{C}\right)^{2}<0.4$ for 
each alloy by using the BCS critical field calculations made by Swenson (128). The average value of $\mathrm{H}_{0}$ determined by this procedure was then taken to be the value of $\mathrm{H}_{0}$ for a particular alloy as shown in Figures 18 and $19 . \mathrm{H}_{0}$ derived in this way is constant within the accuracy. of the data, so a simple BCS extrapolation fits the data rather well. A value of $\mathrm{H}_{0}$ could not be obtained for Th-0.150 at.\% $U$ using this procedure because $T_{C}=0.495 \mathrm{~K}$ and sufficiently low reduced temperatures could not be attained with the $\mathrm{He}^{3}$ evaporation refrigerator. The results of these $\mathrm{H}_{0}$ calculations are given in Appendix B. A BCS critical field curve was then generated for each alloy using the values of $\mathrm{T}_{\mathrm{C}}$ and $\mathrm{H}_{\mathrm{O}}$. Figures 14 and 15 show the excellent agreement between the data and the BCS critical field curves which are indicated by the solid lines.

The critical field curves are also presented in Figures 20-25 as deviations from the fiducial parabola given by Equation 1, where the deviation function, $D(t)$, is given by

$$
D(t)=h-\left(1-t^{2}\right)
$$

Data over the whole temperature range for all the alloys lay within about $0.3 \%$ of the BCS theory, indicated by the solid curves. These results can be contrasted to the behavior of $D(t)$ versus $t^{2}$ for the ThGa alloy system studied by Decker and Finnemore (72) (hereafter referred to as DF). The DF 
THIS PAGE

\section{WAS INTENTIONALLY LEFT BLANK}


Figure 18. Results of the calculations of $\mathrm{H}_{0}$ from the $\mathrm{BCS}$ theory for the ThU samples exhibiting small hysteresis. The bars on the Th-0.030 at. $\% \mathrm{U}$ data show the width of the superconducting transitions. The dark lines indicate the average value of $\mathrm{H}_{0}$ obtained. 


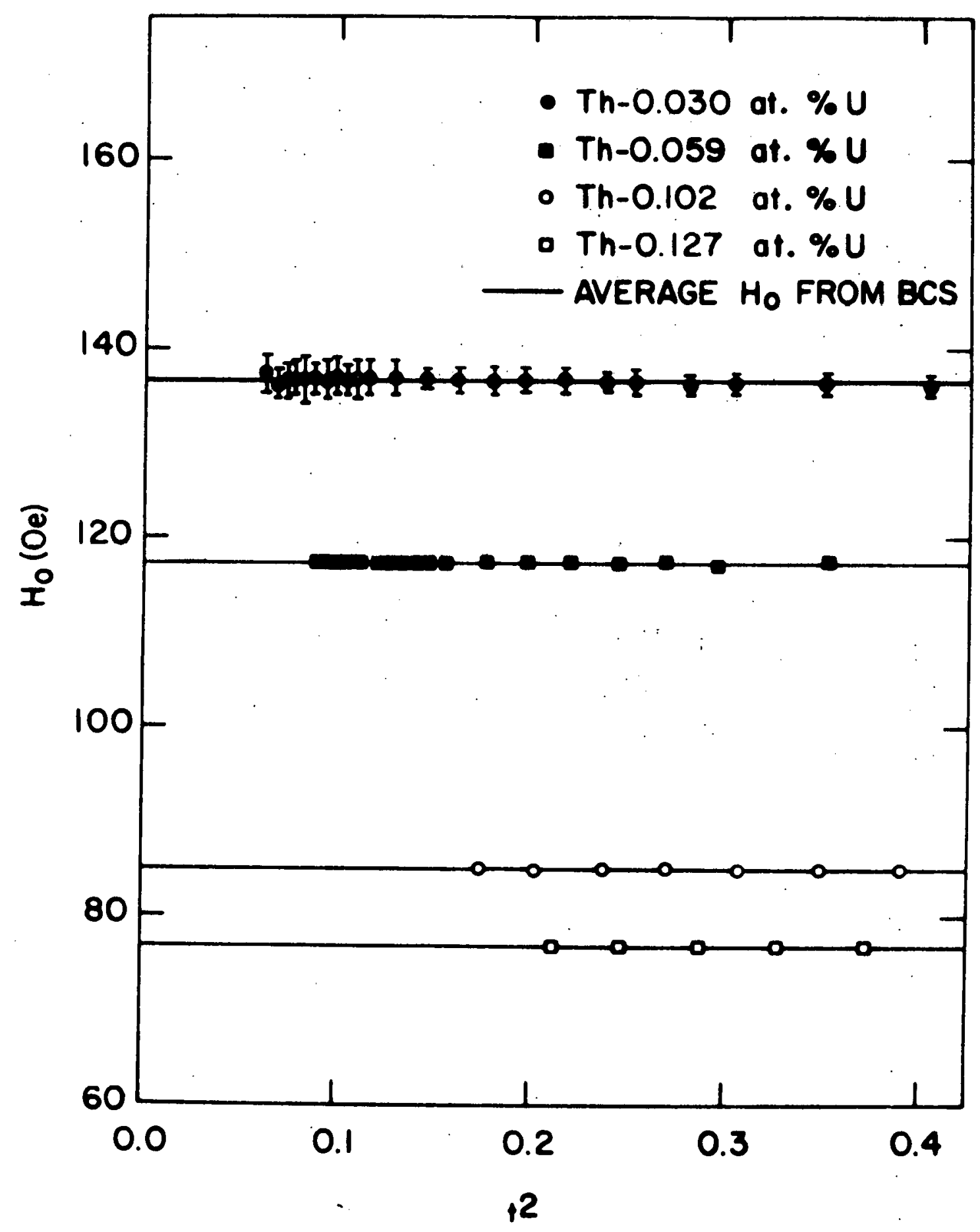


THIS PAGE

WAS INTENTIONALLY

LEFT BLANK 
Figure 19. Results of the calculations of $\mathrm{H}_{\mathrm{O}}$ from the BCS theory for the ThU samples exhibiting large hysteresis. The bars on the data show the width of the superconducting transitions. The dark lines indicate the average value of $\mathrm{H}_{0}$ obtained. 


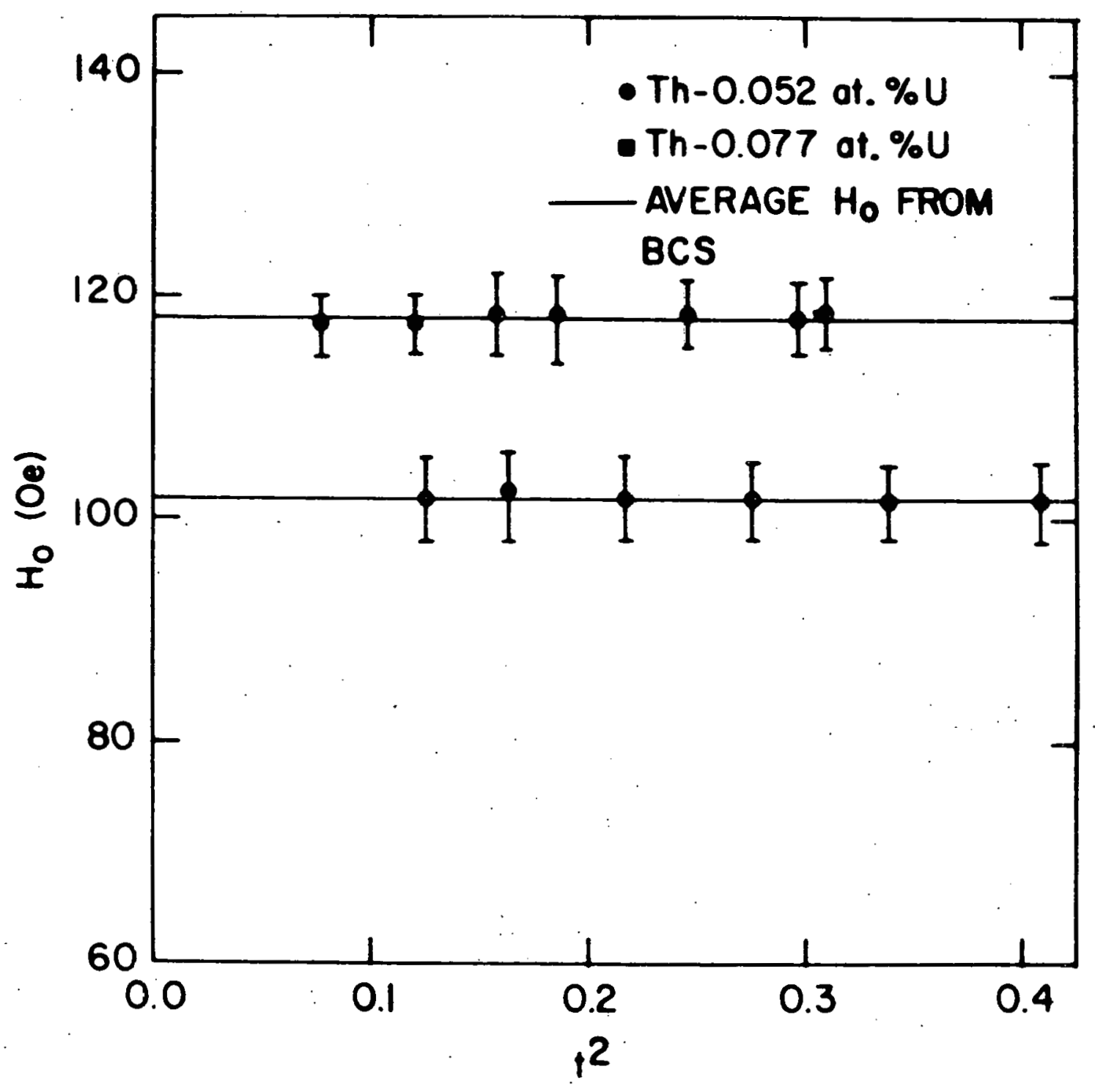




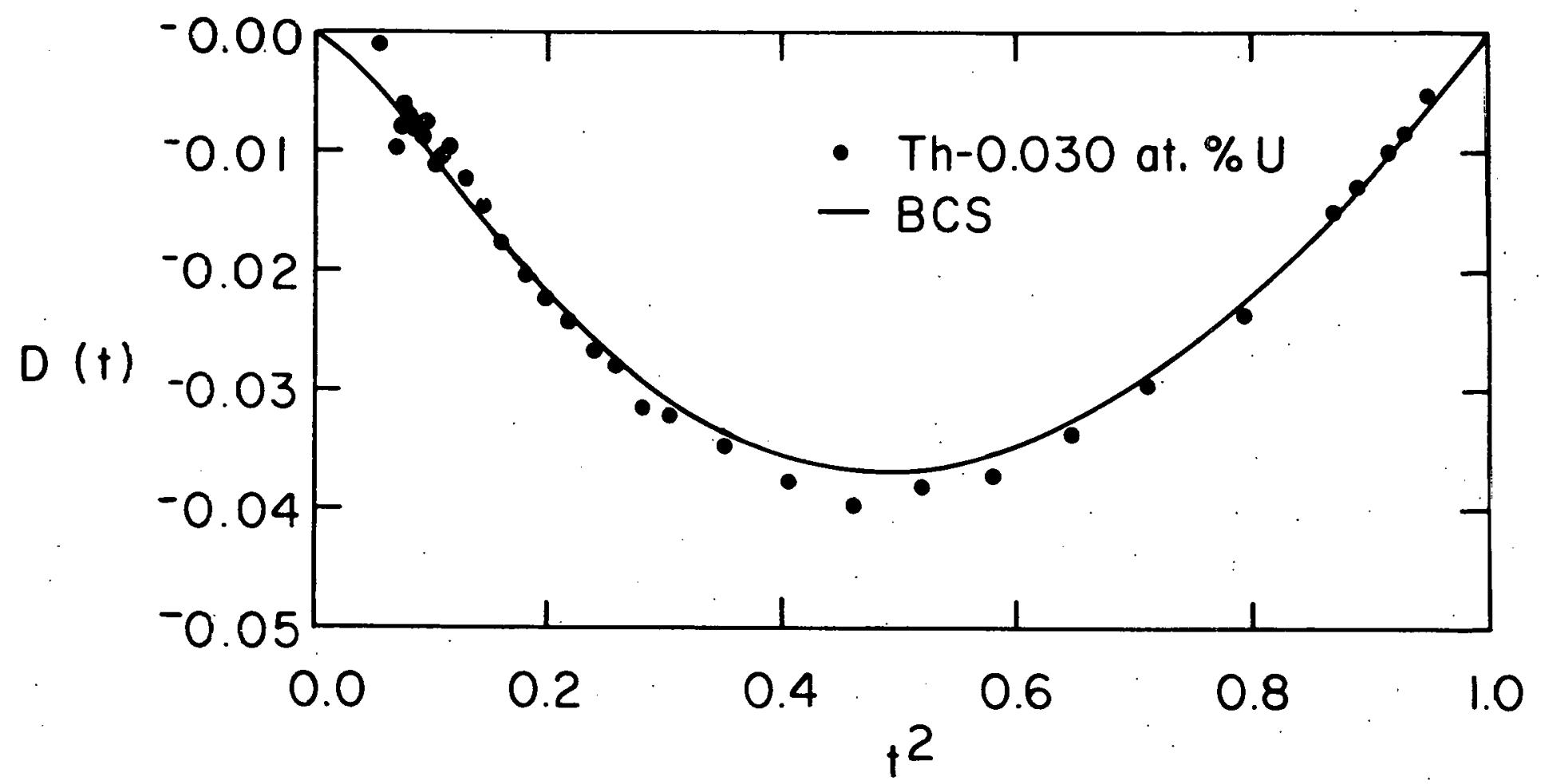

$\vec{\infty}$

Figure 20. Deviation of the Th-0.030 at.\% U critical field curve from the fiducial parabola compared to the BCS theory (solid curve). 


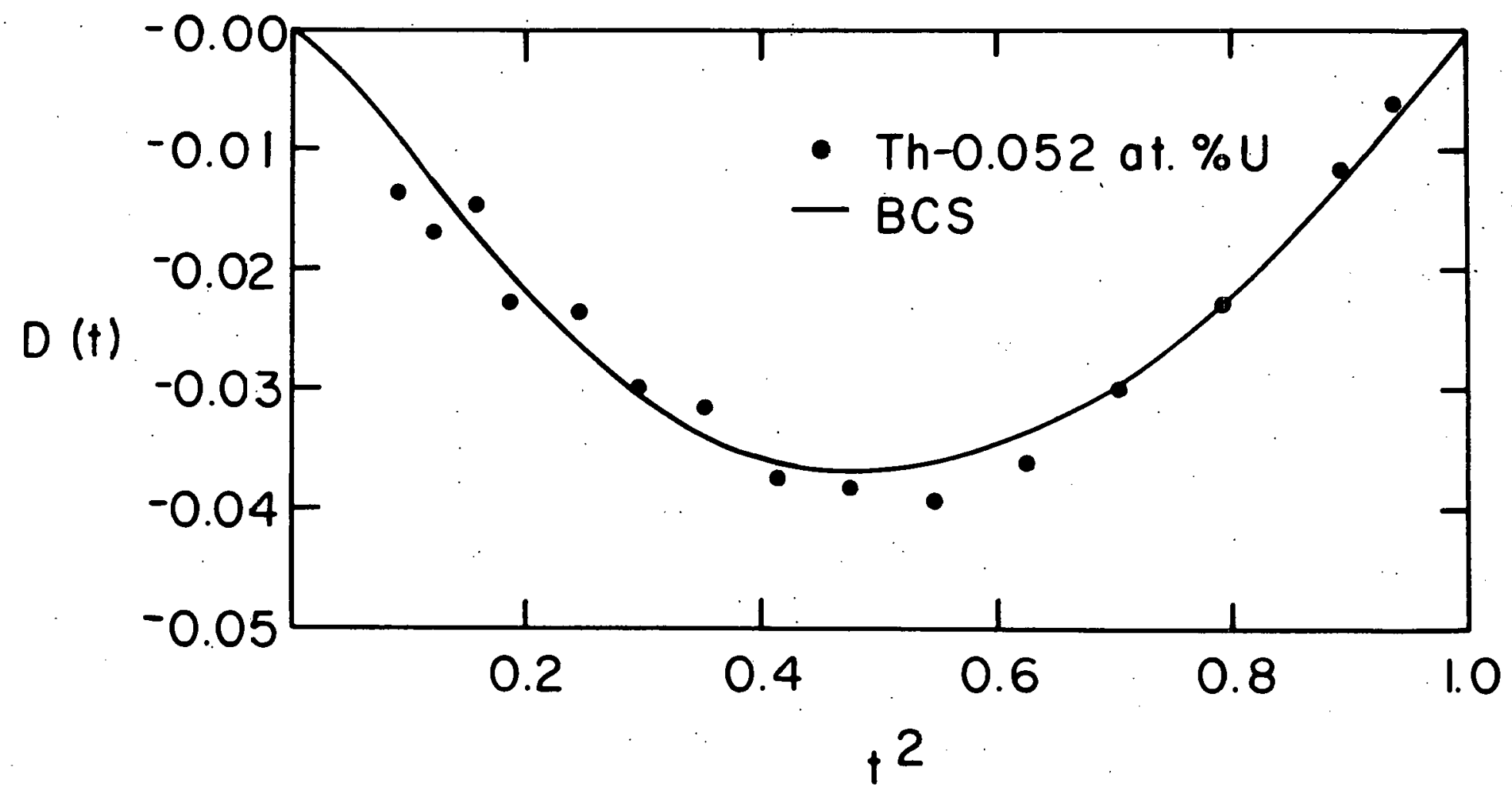

Figure 21. Deviation of the Th-0.052 at.\% U critical field curve from the fiducial parabola compared to the BCS theory (solid curve). 


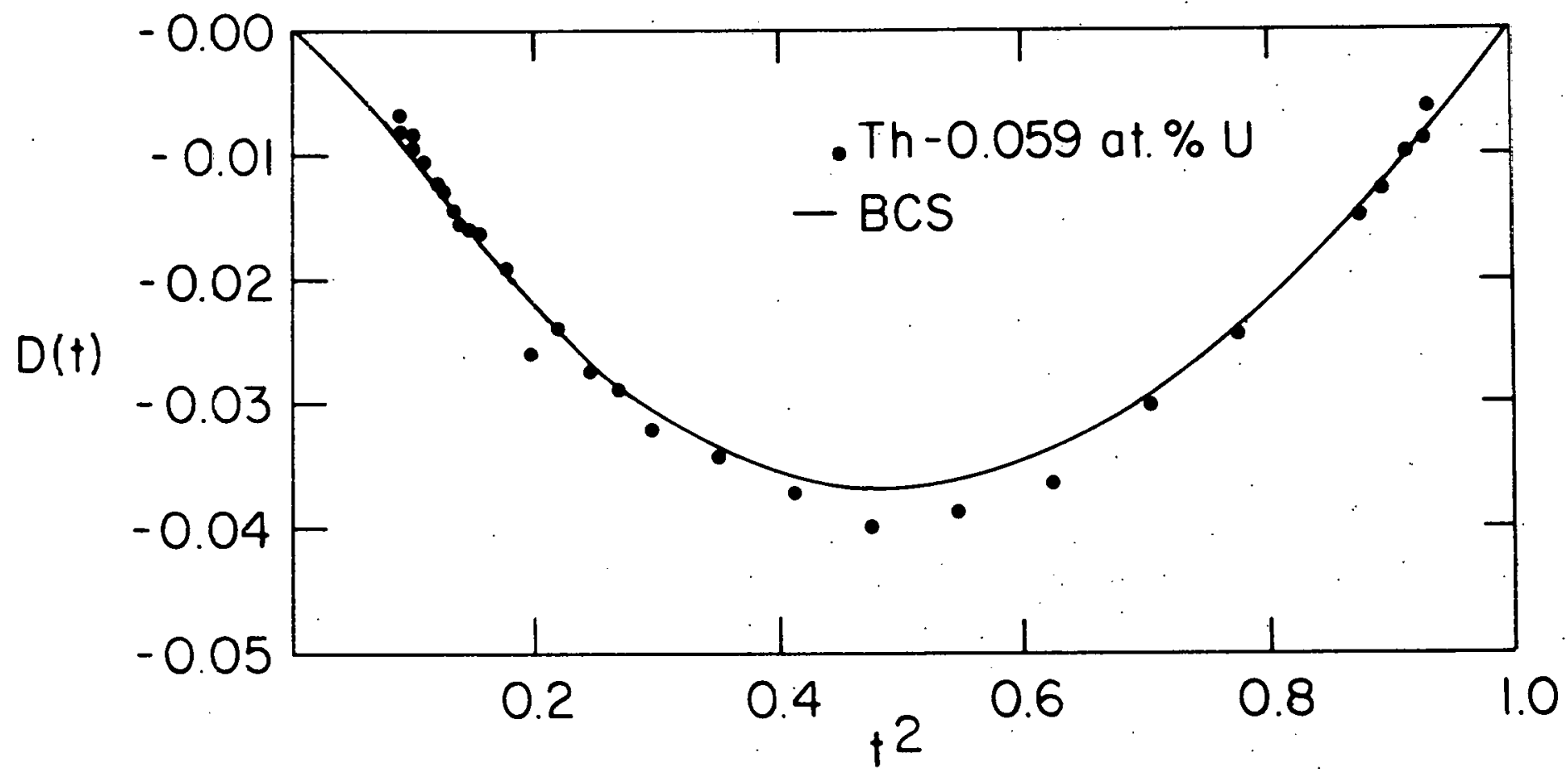

Figure 22. Deviation of the Th- 0.059 at.\% U critical field curve from the fiducial parabola compared to the BCS theory (solid curve). 


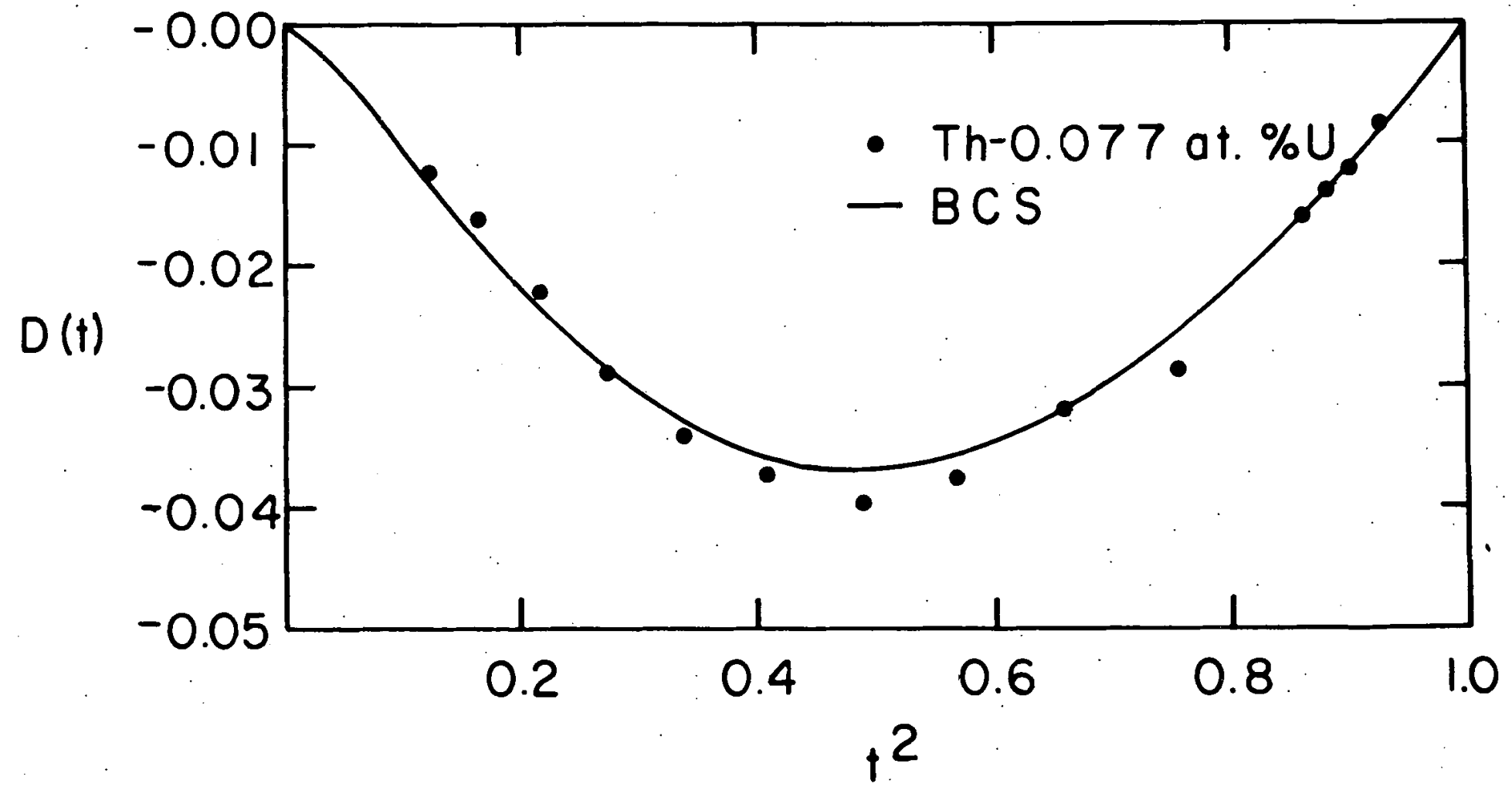

$\stackrel{\infty}{\mapsto}$

Figure 23. Deviation of the Th-0.077 at.\% U critical field curve from the fiducial parabola compared to the BCS theory (solid curve). 


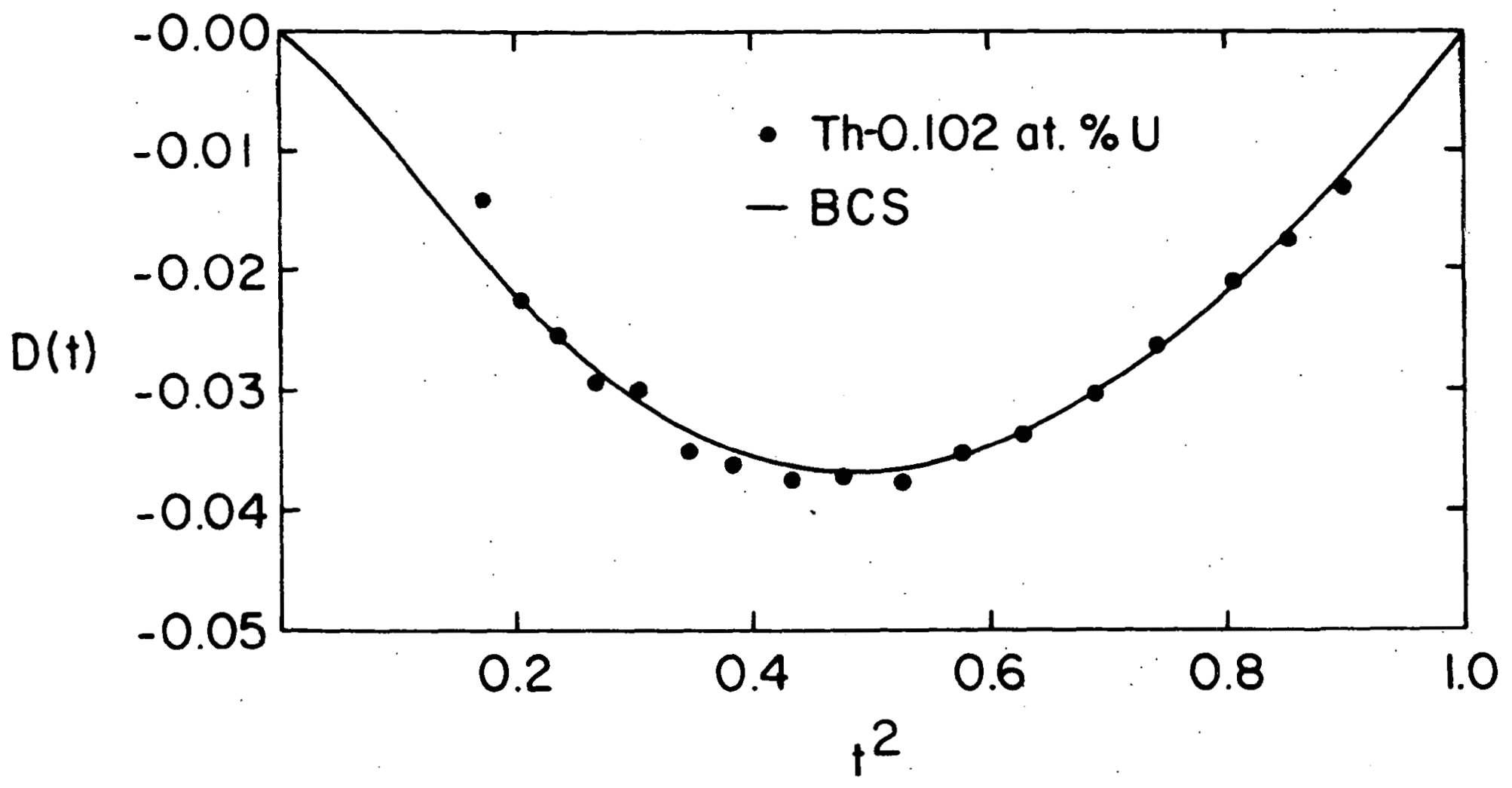

Figure 24. Deviation of the Th-0.102 at.\% U critical field curve from the fiducial parabola compared to the BCS theory (solid curve). 


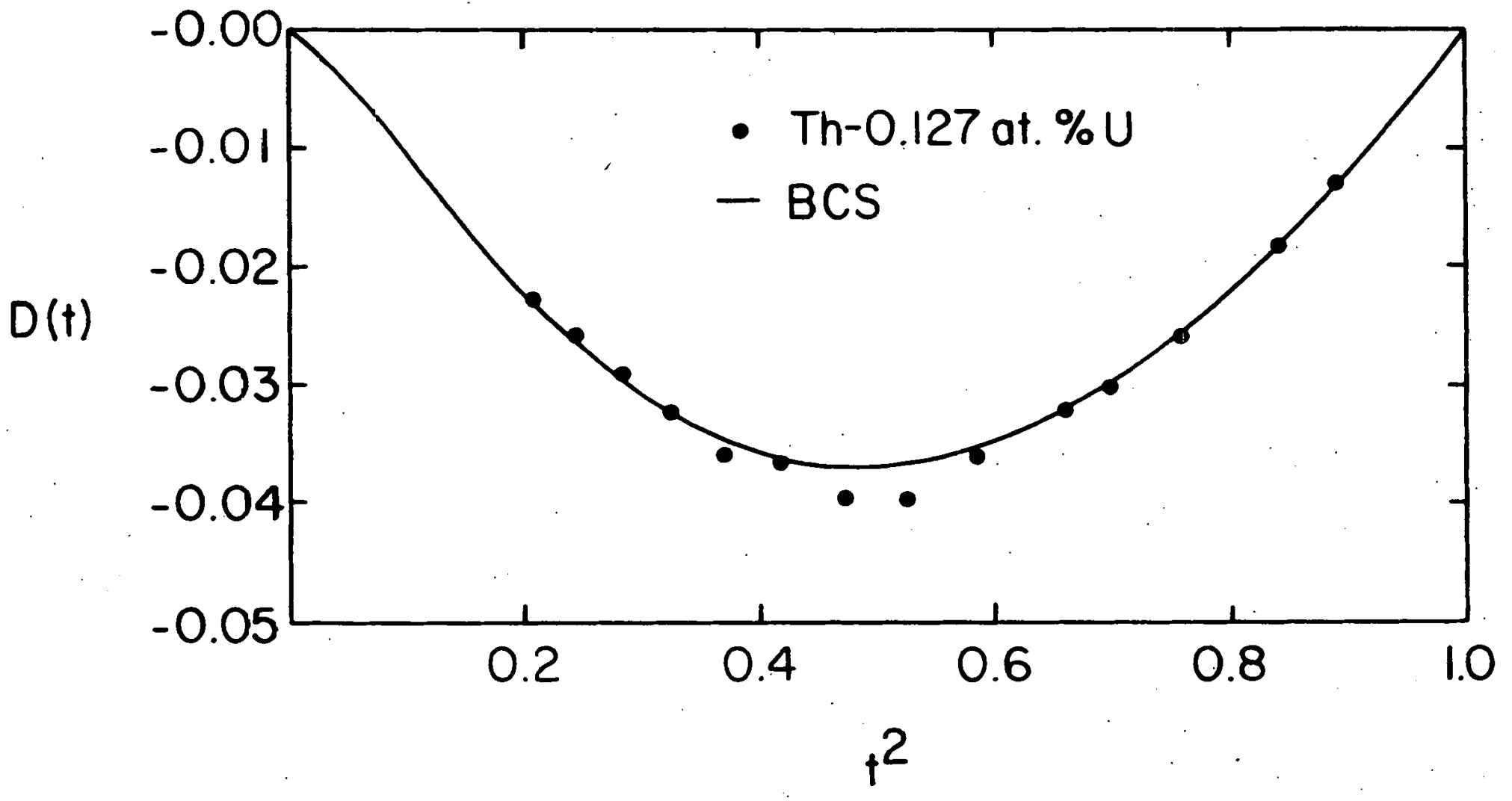

Figure 25. Deviation of the Th-0.127 at.\% U critical field curve from the fiducial parabola compared to the BCS theory (solid curve). 
data are presented in Figure 26 and compared to the BCS and AG theories, indicated by the dashed curves and solid curves, respectively. The ThGd data lie considerably below the BCS theory curves and are in close agreement with the AG theory curves.

The electronic specific heat coefficient, $\gamma$, was also calculated from simple BCS using the relation (35)

$$
Y=\mathrm{H}_{0}^{2} / 8 \pi(0.2364) \mathrm{T}_{\mathrm{C}}{ }^{2}=0.168 \frac{\mathrm{H}_{\mathrm{O}}{ }^{2}}{\mathrm{~T}_{\mathrm{C}}{ }^{2}} \mathrm{~V}_{\mathrm{M}}
$$

where $\mathrm{V}_{\mathrm{M}}$ is the molar volume. Using Equation 54 with $\mathrm{V}_{\mathrm{M}}=19.7 \mathrm{~cm}^{3} / \mathrm{mole}(117), \mathrm{T}_{\mathrm{C}}=1.390 \mathrm{~K}$, and $\mathrm{H}_{0}=159.22 \mathrm{Oe}$ for a pure Th sample, DF found that $\gamma=4.34 \mathrm{~mJ} / \mathrm{mole}-\mathrm{K}^{2}$, in close agreement with the calorimetric value of $4.31 \mathrm{~mJ} / \mathrm{mole}$ $\mathrm{K}^{2}$ (129). The values of $\mathrm{T}_{\mathrm{C}}$ and $\mathrm{H}_{\mathrm{O}}$ used by $\mathrm{DF}$ were obtained by the same method discussed above for determining $\mathrm{T}_{\mathrm{C}}$ and $\mathrm{H}_{\mathrm{O}}$ for the ThU alloys. The results of the $\gamma$ calculations for all the alloys are presented in Figure 27, where $\gamma$ is plotted versus uranium concentration. The same data are also displayed in Figure 28 except the samples with large hysteresis have been excluded. Also included in these figures are calurimetric $\gamma$ values for Thu by Luengo et al. (113) and the pure thorium $Y$ of DF. An examination of the data indicates that $\gamma$ values calculated from simple BCS are in reasonable agreement with the calorimetric $\gamma$ values but 


\section{THIS PAGE}

\section{WAS INTENTIONALLY LEFT BLANK}


Figure 26. Deviations of the ThGa critical field curves from the fiducial parabola compared to the BCS theory (dashed curves) and the $A G$ theory (solia curves) from Decker and Finnemore (7.2): 

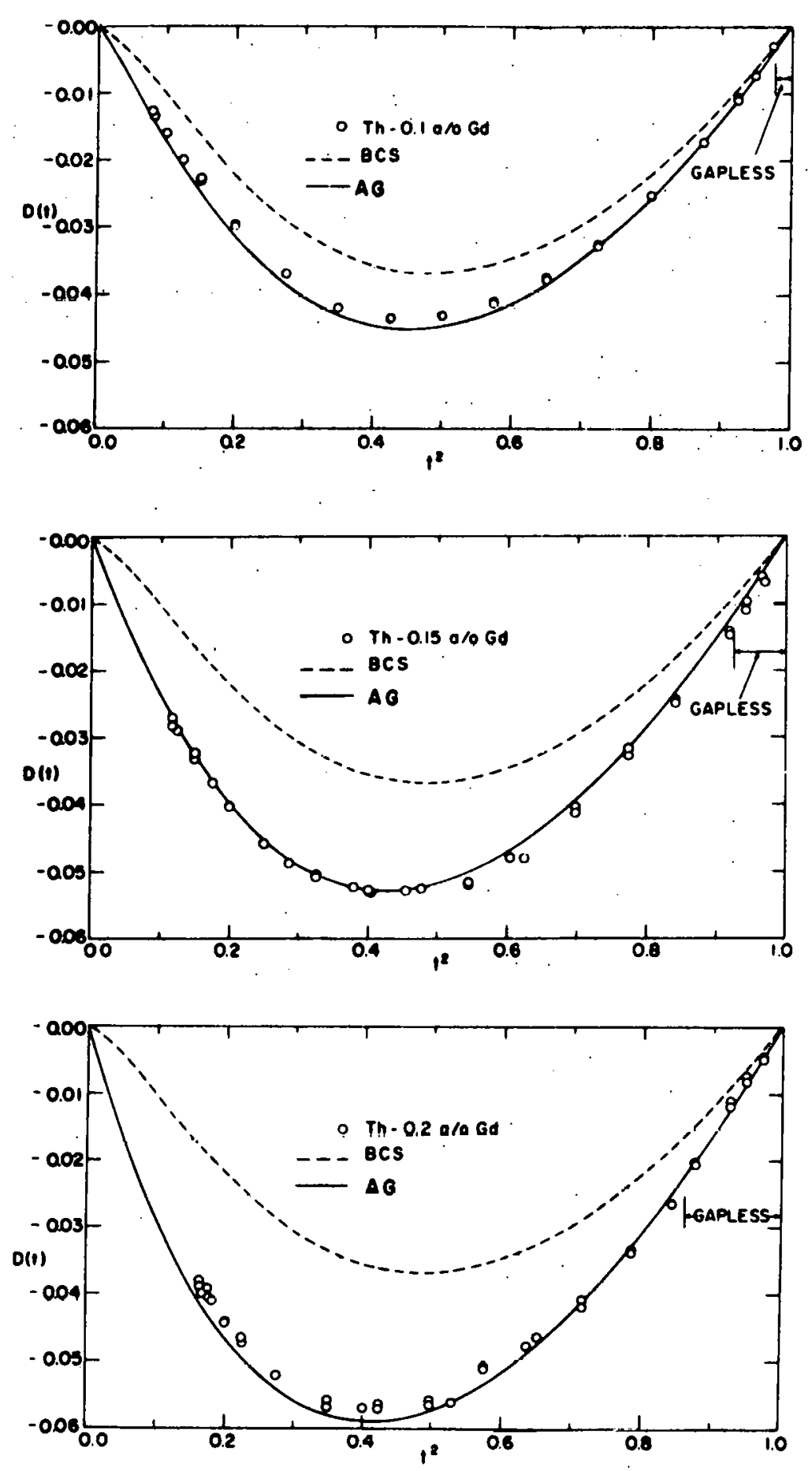


\section{THIS PAGE}

\section{WAS INTENTIONALLY LEFT BLANK}


Figure 27. The electronic specific heat coefficient, $Y$, versus $U$ concentration in ThU obtained from this study (solid.squares) and from the calorimetric study (open circles) by Luengo et al. (113). The value of $\gamma$ obtained for pure Th by Decker and Finnemore $(72)$ is also show (solid circle). 


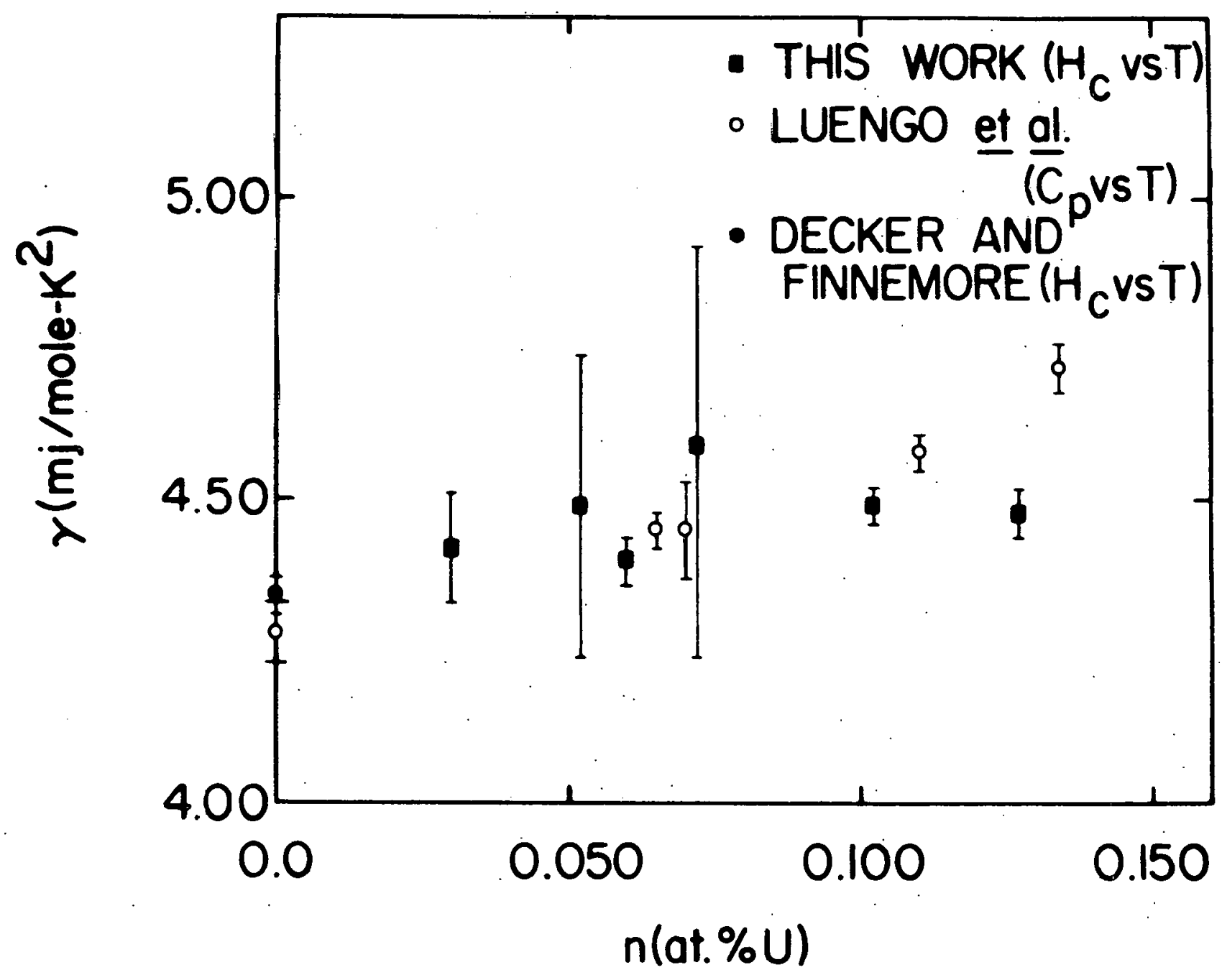


THIS PAGE

WAS INTENTIONALLY

LEFT BLANK 
Figure 28. The electronic specific heat coefficient, $Y$, versus $U$ concentration for ThU obtained from this study for the samples exhibiting small hysteresis (solid squares) and from the calorimetric study (open circles) by Luengo et al. (113). The value of $\gamma$ obtained for pure Th by Decker and Finnemore (72) is also shown (solid circle). 


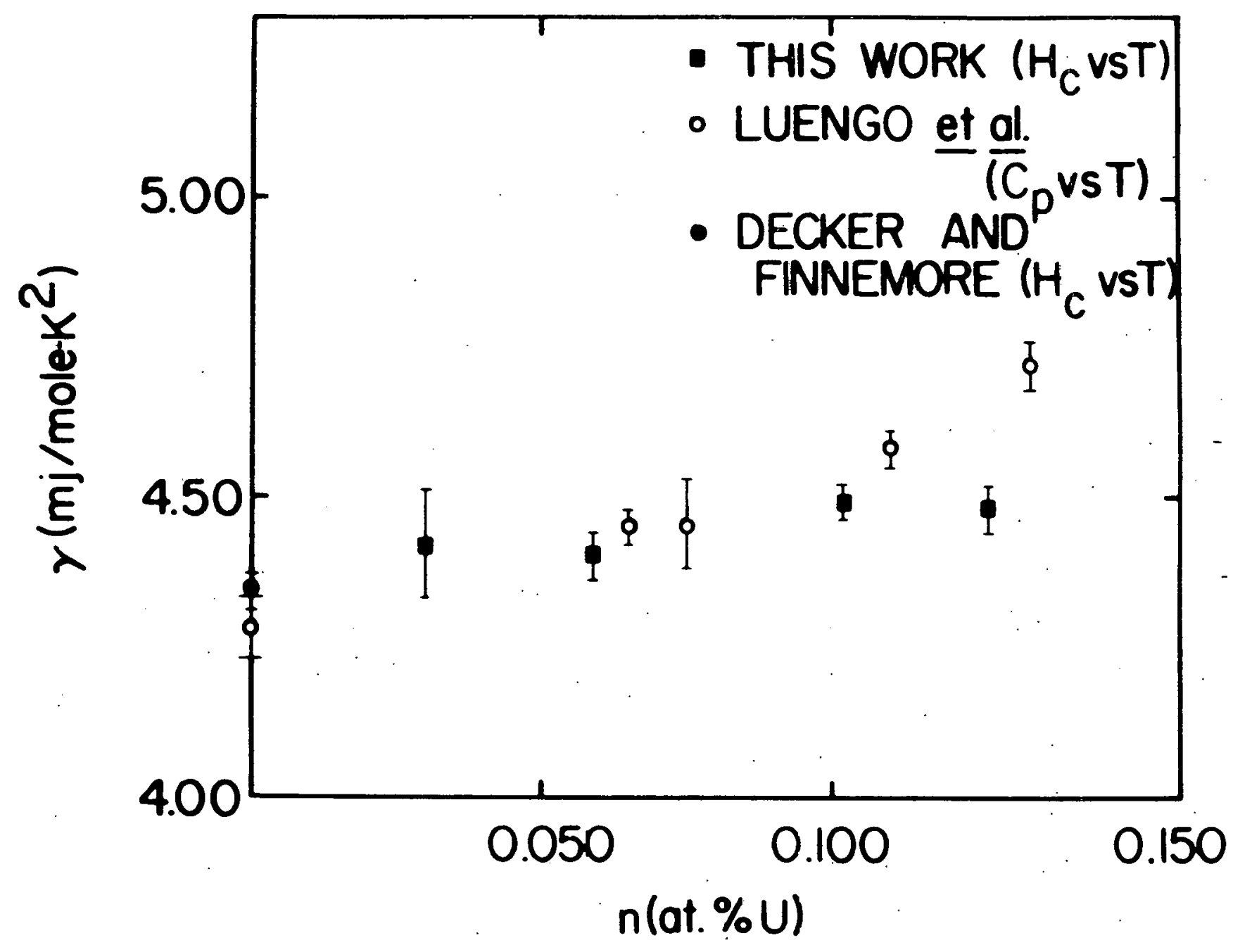


tend to iie somewhat lower if the samples with large hysteresis are excluded as in Figure 28. The general trend of these data indicates that $y$ is enhanced at a rate of about $2 \mathrm{~mJ} / \mathrm{mole}-\mathrm{K}^{2}$ at.\% U. If $\mathrm{it}$ is assumed that the increase in $Y$ with $U$ concentration is due to uranium $5 f$ resonant states, the local density of states at the Fermi level, for one spin direction, contributed by each $U$ atom would be about 42 states/eV-atom, a rather large number.

The reduced specific heat jumps, $\left(\Delta \mathrm{C} / \Delta \mathrm{C}_{\mathrm{p}}\right)_{\mathrm{T}_{\mathrm{c}}}$, are plotted versus the reduced transition temperatures in Figure 29. The values of $(\Delta \mathrm{C})_{\mathrm{T}_{\mathrm{C}}}$ were calculated by first fitting the data near $\mathrm{T}_{\mathrm{C}}$ to $\mathrm{H}_{\mathrm{C}}=\mathrm{A}+\mathrm{BT}^{2}$ and then using Rutger's formula (Equation 15). The value of $\left(\Delta C_{p}\right)_{T_{C}}$ was obtained by extrapolating the $(\Delta \mathrm{C})_{\mathrm{T}_{\mathrm{C}}}$ data to $\mathrm{T} / \mathrm{T}_{\mathrm{CP}}=1$, and this procedure yielded a value of $\left(\Delta c_{p}\right)_{T_{c}}=8.75 \pm 0.25 \mathrm{~mJ} / \mathrm{mole}-\mathrm{K}^{2}$, in good agreement with $\left(\Delta \mathrm{C}_{\mathrm{p}}\right)_{\mathrm{T}}=8.5 \pm 0.2 \mathrm{~mJ} / \mathrm{mole}-\mathrm{K}^{2} \mathrm{ob}-$ tained by Luengo et al. (113) and the value of $\left(\Delta \mathrm{C}_{\mathrm{p}}\right)_{\mathrm{T}_{\mathrm{C}}}=$ $9.0 \mathrm{~mJ} / \mathrm{mole}-\mathrm{K}^{2}$ obtained by $\mathrm{DF}$. The bars on the data points indicate the uncertainties in the slopes of the critical field curves near $T_{C^{*}}$ BCS (31) give the relation

$$
\frac{(\Delta \mathrm{C})_{\mathrm{T}_{\mathrm{C}}}}{\gamma^{\mathrm{T}_{\mathrm{C}}}}=1.43 \text {, }
$$

and if $Y$ is assumed to remain constant, then Equation 55 gives 


\section{THIS PAGE}

WAS INTENTIONALLY

\section{LEFT BLANK}


Figure 29. Reduced specific heat jump, $\left(\Delta \mathrm{C} / \Delta \mathrm{C}_{\mathrm{p}}\right)_{\mathrm{T}_{\mathrm{C}}}$, versus $\mathrm{T}_{\mathrm{C}} / \mathrm{T}_{\mathrm{Cp}}$. The dashed and the dot-dashed lines represent the BCS theory results, and the solid line is the AG theory result. The values obtained in this study of ThU are shown by the dark circles, while the values obtained by Luengo et al. (113) are shown by the open circles. Also included are results, for ThGd (solid squares) by Decker and Finnemore (72) and for ( $\mathrm{La}, \mathrm{Ce}) \mathrm{Al}_{2}$ (solid triangles) by Luengo, Maple, and Fertig (87). 


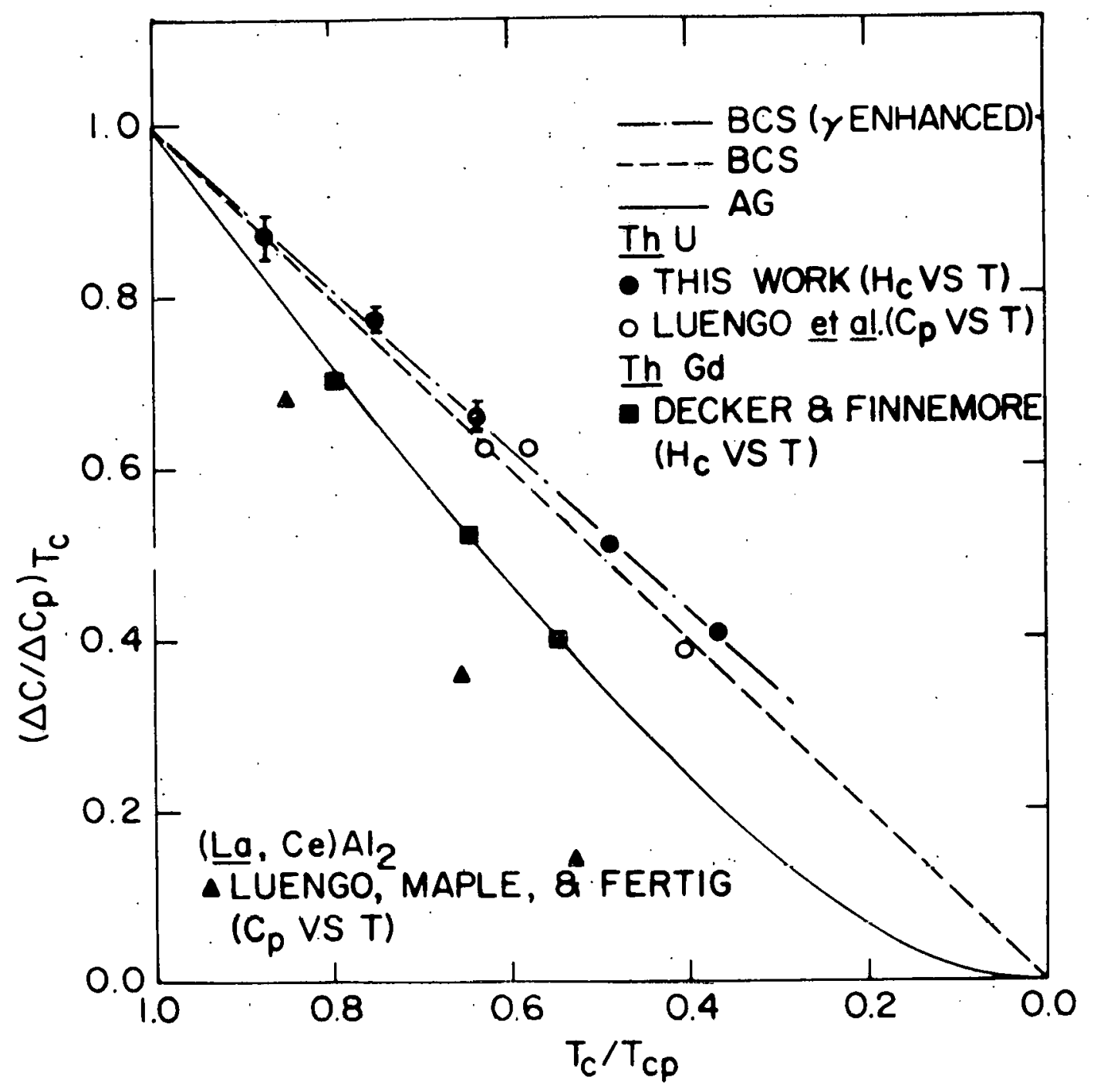




$$
\left(\Delta C / \Delta C_{p}\right)_{T}=T_{C} / T_{C p}
$$

and one obtains the dashed line shown in Figure 29. If, however, $\gamma$ changes, as $\mathrm{T}_{\mathrm{C}}$ changes, as would be expected from Figure 28, then Equation 55 becomes

$$
\left(\Delta \mathrm{C} / \Delta \mathrm{C}_{\mathrm{p}}\right)_{\mathrm{T}_{\mathrm{c}}}=Y / Y_{\mathrm{p}} \mathrm{T}_{\mathrm{C}} / \mathrm{T}_{\mathrm{Cp}}
$$

If it is assumed that $y$ is enhanced at a constant rate of about $2.0 \mathrm{~mJ} / \mathrm{mole}-\mathrm{K}^{2}$ at. $\% \mathrm{U}$, then the dot-dashed line shown in Figure 29 is obtained. It is seen that the Thu data from both this study and Luengo et al. (113) lie close to the predictions of BCS theory given either by Equation 56 or Equation 57. Also shown in Figure 29 are the reduced specific heat jumps for ThGd (72) which closely follow the AG theory indicated by the solid curve, and the reduced specific heat jumps for the superconducting Kondo alloy system $(\underline{L a}, \mathrm{Ce}) \mathrm{Al}_{2}$ (87), which lie considerably below the AG theory result.

Measured and calculated values (using simple BCS) of various sample characteristics for the ThU alloys used in this study are given in Table 2. An anomaly which appears in the data is that the values of $\mathrm{T}_{C}$ for $\mathrm{Th}-0.052$ at.\% $\mathrm{U}$ and Th-0.059 at.\% U are almost the same, even though the chemical analysis of these alloys indicated significantly different uranium concentrations. As noted above, however, the 
Table 2. Measured and calculated (from simple BCS) values for ThU alloys

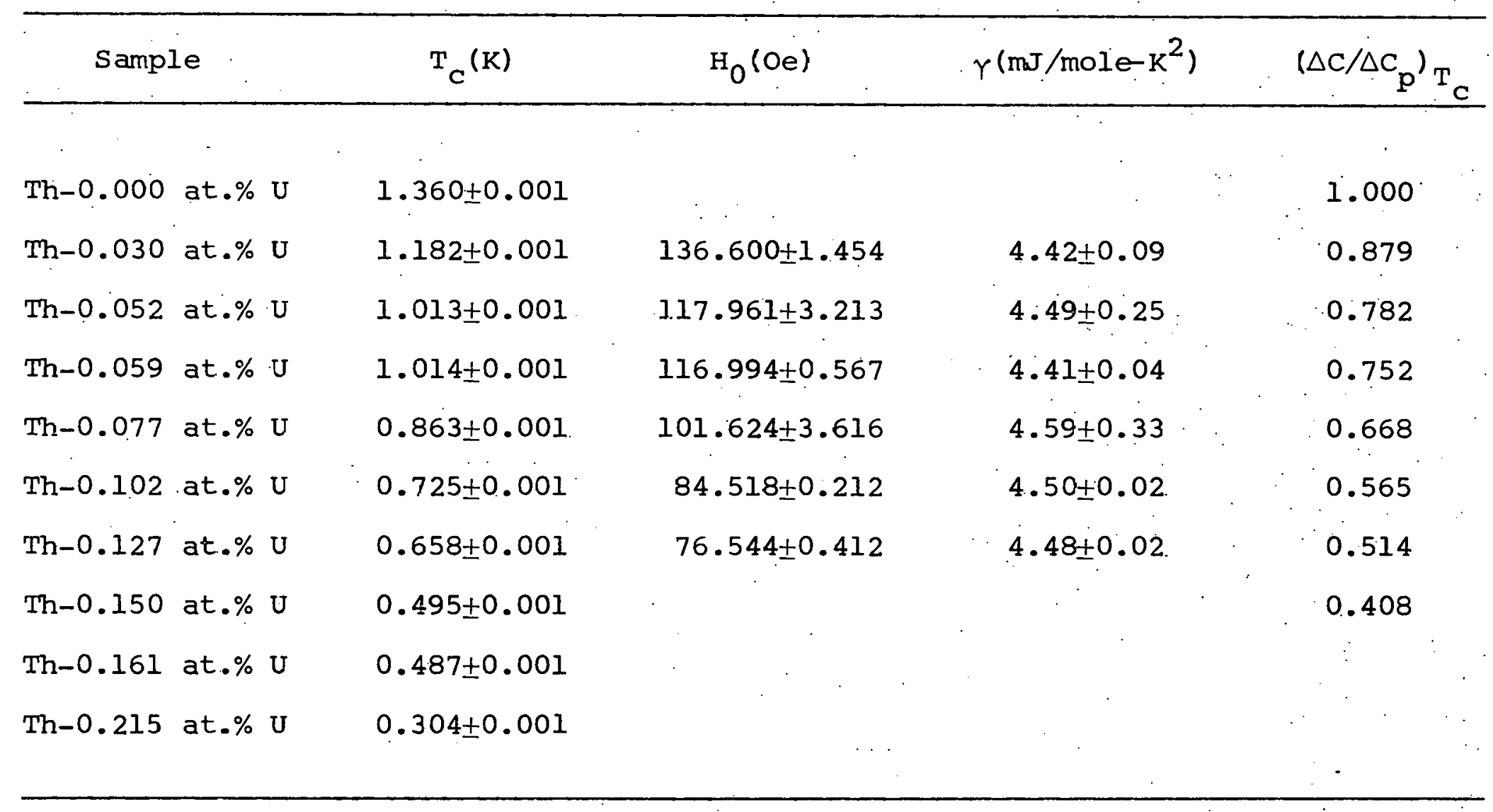


lower temperature superconducting transitions for the Th-0.052 at.\% U sample exhibited large hysteresis and this could imply that the uranium atoms in this sample were not homogeneous. Because only a small portion of each sample was cut off and analyzed, clustering of uranium atoms in that portion could give a higher uranium concentration than actually exists in the bulk specimen. It should also be noted that the uranium concentrations of the ThU alloys reported in the specific heat study of Luengo et al. (113) are highly suspect because the $T_{C}$ values differ significantly from those found by Maple et al. (110) and from those reported here. For example, Luengo et al. reported that no superconducting transition could be observed in their $\mathrm{Th}-0.134$ at.\% $\mathrm{U}$ sample for temperatures as low as $\mathrm{T} \simeq 0.3 \mathrm{~K}$, whereas Maple et al. (110) and this work found that a nominal Th-0.2 at.\% $\mathrm{U}$ sample undergoes a superconducting transition above $0.3 \mathrm{~K}$. Also Luengo et al. reported $\mathrm{T}_{\mathrm{C}}=0.550 \mathrm{~K}$ for Th-0.110 at.\% $U$, which is significantly lower than the value of $T_{C}=0.650 \mathrm{~K}$ for $T_{h}-0.127$ at. $\% \mathrm{U}$ found in this study.

Before proceeding to the details of the final fitting of the critical field curve data to take into account anisotropy effects, we shall first provide a bit of theoretical background. Markowitz and Kadanoff (130) (hereafter referred to as MK) worked out the details of the Anderson anisotropy 
idea (63) discussed earlier using a model in which the constant effective electron-electron interaction $V^{\prime}$ in the simple BCS model is replaced by the effective electron-electron matrix element

$$
v_{p p^{\prime}}^{\prime}=\left(1+a_{p}\right) v^{\prime}\left(1+a_{p}^{\prime}\right)
$$

where $a_{p}$ is an anisotropy function chosen to have zero average over the Fermi surface. MK calculated the change in $\mathrm{T}_{\mathrm{C}^{\prime}} \delta \mathrm{T}_{\mathrm{C}^{\prime}}$ for a pure superconductor containing simple nonmagnetic impurities and found that

$$
\delta \mathrm{T}_{\mathrm{c}}(x)=\mathrm{K}^{\mathrm{i}} \mathrm{X}+\left\langle\mathrm{a}^{2}\right\rangle \mathrm{T}_{\mathrm{c}} \mathrm{I}_{\mathrm{c}}(x),
$$

where $X$ is proportional to the impurity density, $x^{i}$ is a constant dependent on the impurity and the host, $\left\langle a^{2}\right\rangle=$ $\left\langle a_{p}^{2}\right\rangle$ av. is the mean-squared anisotropy, and $I_{c}$ is a function obtained by numerical integration. The first term in Equation 59 is the valence effect, while the second term is the anisotropy effect. In the experimental regions of interest, MK showed that $I_{C}$ could be approximated by

$$
I_{c} \simeq 0.78 \times \ln x-0.36 \times \text {. }
$$

Both $X$ and the residual resistivity ratio, $P_{r^{\prime}}$ defined by

$$
\rho_{r}=\frac{R_{4.2 K}}{R_{273 K}-R_{4.2 K}} \text {, }
$$


where $R_{4.2} \mathrm{~K}$ and $R_{273 \mathrm{~K}}$ are the resistances of the alloy at $4.2 \mathrm{~K}$ and $273 \mathrm{~K}$, respectively, are proportional to the impurity concentration. Hence, MK write

$$
x=\alpha^{i} p_{r},
$$

where $\alpha^{i}$ is a constant of proportionality. Equation 59 can then be written in the form

$$
\delta T_{c}=A p_{r}+B \rho_{r} \ln p_{r}
$$

where $A$ and $B$ are arbitrary constants.

Clem (40) extended the MK model to study the changes in the critical field curves of weak-coupling superconductors due to the presence of anisotropy in the energy gap. The second paper given in reference 40 (hereafter referred to as Clem II) is of particular interest here, because Clem II describes the detailed shape of the critical field curve, $\mathrm{H}_{C}(T)$, in terms of the reduction of the effect due to anisotropy when simple nonmagnetic impurities are added to a pure superconductor. $\mathrm{Clem}$ II shows that the reduced critical field, $h(t)=\mathrm{H}_{C}(t) / \mathrm{H}_{0}$, and the term $\tilde{\mathrm{H}}_{\mathrm{C}^{\prime}}$, defined by

$$
\tilde{\mathrm{H}}_{\mathrm{C}}^{2} \equiv \mathrm{H}_{\mathrm{C}}^{2} / 8 \pi \gamma \mathrm{T}_{\mathrm{C}}^{2}
$$

should be nearly free from valence effects and, therefore, are the relevant quantities when considering changes due to anisotropy. The results are formulated, then, in terms of 
$h, \tilde{H}_{C^{\prime}} t$, and two additional parameters, $\lambda=X / 2 \pi$ and the function $\mathrm{x}_{\mathrm{H}}(t, \lambda)$ used to describe the effectiveness of anisotropy corrections to $\tilde{\mathrm{H}}_{\mathrm{C}}^{2}$.

Two different methods have been used to evaluate $\left\langle\mathrm{a}^{2}\right\rangle$ for pure Th. Anderson et al. (131) found that $\left\langle\mathrm{a}^{2}\right\rangle=$ 0.021 by measuring the depression of $\mathrm{T}_{C}$ of pure $\mathrm{Th}_{\mathrm{i}}$ as carbon impurities were added. Gubser (132), on the other hand, obtained a value of $\left\langle a^{2}\right\rangle=0.019$ using the slope of the critical field curve at $\mathrm{T}_{\mathrm{C}}$ of the pure Th sample of $\mathrm{DF}$. A value of $\left\langle a^{2}\right\rangle=0.02$ will be used here.

Figure 30 shows the deviation function obtained by DF for pure Th compared to the simple BCS theory (solid curve), and to the clem theory (dot-dashed curve) for a pure superconductor with $\left\langle\mathrm{a}^{2}\right\rangle=0.02$. On this figure it can be seen that the $D(t)$ data lie closer to the BCS theory curve than to the clem theory curve, but this is a bit misleading because, as noted above, the $\mathrm{H}_{0}$ obtained by $\mathrm{DF}$ for pure Th was calculated from the simple BCS theory which neglects anisotropy corrections. A more accurate calculation of $\mathrm{H}_{0}$ should include anisotropy corrections in the extrapolation. Similarly, the $\gamma$ value obtained by DF also needs to include anisotropy rorrections.

In view of the above arguments, we have reanalyzed the DF pure Th data. The value of $\lambda$ for the DF pure Th sample is essentially zero, so the low temperature data of DF were 
100

THIS PAGE

\section{WAS INTENTIONALLY \\ LEFT BLANK}


Figure 30. Deviation function for pure thorium from Decker and Finnemore (72). The BCS theory result is indicated by the solid curve. The dot-dashed curve is the clem theory result for a pure superconductor with $\left\langle\mathrm{a}^{2}\right\rangle=0.02$. 


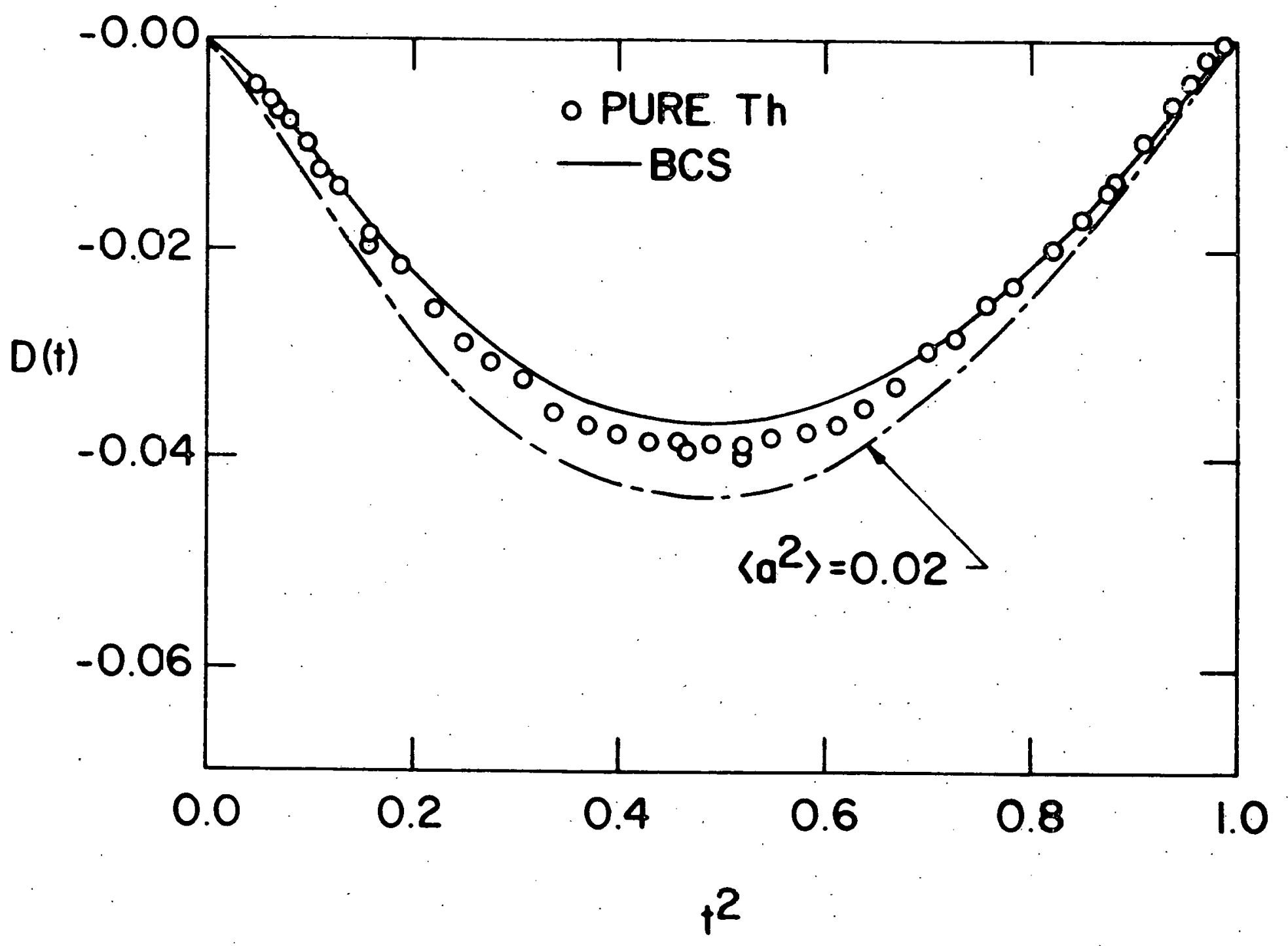


fitted to the clem II relation

$$
h(t, \lambda)=h^{0}(t)\left\{I-\left\langle a^{2}\right\rangle\left[x_{H}(t, \lambda)-x_{H}(0, \lambda)\right]\right\} .
$$

with $\lambda=0$. The $h^{0}(t)$ in Equation 65 are the simple BCS critical field values and may be obtained from Swenson's tables $(128) .\left\langle a^{2}\right\rangle$ is taken to be 0.02 and $x_{H}(0,0)=1$. Using the Clem II notation, $\mathrm{X}_{\mathrm{H}}(t, 0)$ is given by

$$
X_{H}(t, 0)=\left[\frac{\varepsilon_{O}(t)}{\varepsilon_{0}(0)} \frac{H_{O}}{H_{C}(t)}\right]^{2} \frac{\Lambda(0)}{\Lambda(t)},
$$

where $\varepsilon_{0}(t)$ and $\Lambda(t)$ are the energy gap and penetration depth, respectively, of a simple BCS superconductor. values of $\varepsilon_{0}(t) / \varepsilon_{0}(0)$ and $\Lambda(0) / \Lambda(t)$ may be obtained from Mühlschelegel's tables (35). The value of $\mathrm{H}_{0}$ calculated by this procedure is 159.73 Oe, which is about 0.5 Oe larger than the value obtained by DF. This anisotropy corrected $\mathrm{H}_{0}$ was then used to calculate $\mathrm{D}(t)$. The results of this calculation are shown in Figure 31, where the $x^{\prime}$ s indicate the anisotropy corrected $D(t)$ values. The anisotropy corrected data now lie closer to the clem theory curve (dotdashed curve) than to the simple BCS theory curve (solid curve).

An anisotropy corrected $\gamma$ for pure Th may now be obtained from the clem II relation

$$
\tilde{\mathrm{H}}_{\mathrm{O}}^{2}(\lambda)=\tilde{\mathrm{H}}_{\mathrm{O}}^{2}\left[1-2\left\langle\mathrm{a}^{2}\right\rangle \mathrm{X}_{\mathrm{H}}(0, \lambda)\right] \text {, }
$$




\section{THIS PAGE}

WAS INTENTIONALLY

LEFT BLANK 
Figure 31. Deviation function for pure thorium using both the simple BCS theory $\mathrm{HO}_{0}$ (open circles) and the anisotropy corrected $\mathrm{H}_{0}\left(\mathrm{x}^{\prime} \mathrm{s}\right)$. The BCS theory result is indicated by the solid curve. The dotdashed curve is the clem theory result for a pure superconductor with $\left\langle a^{2}\right\rangle=0.02$. 


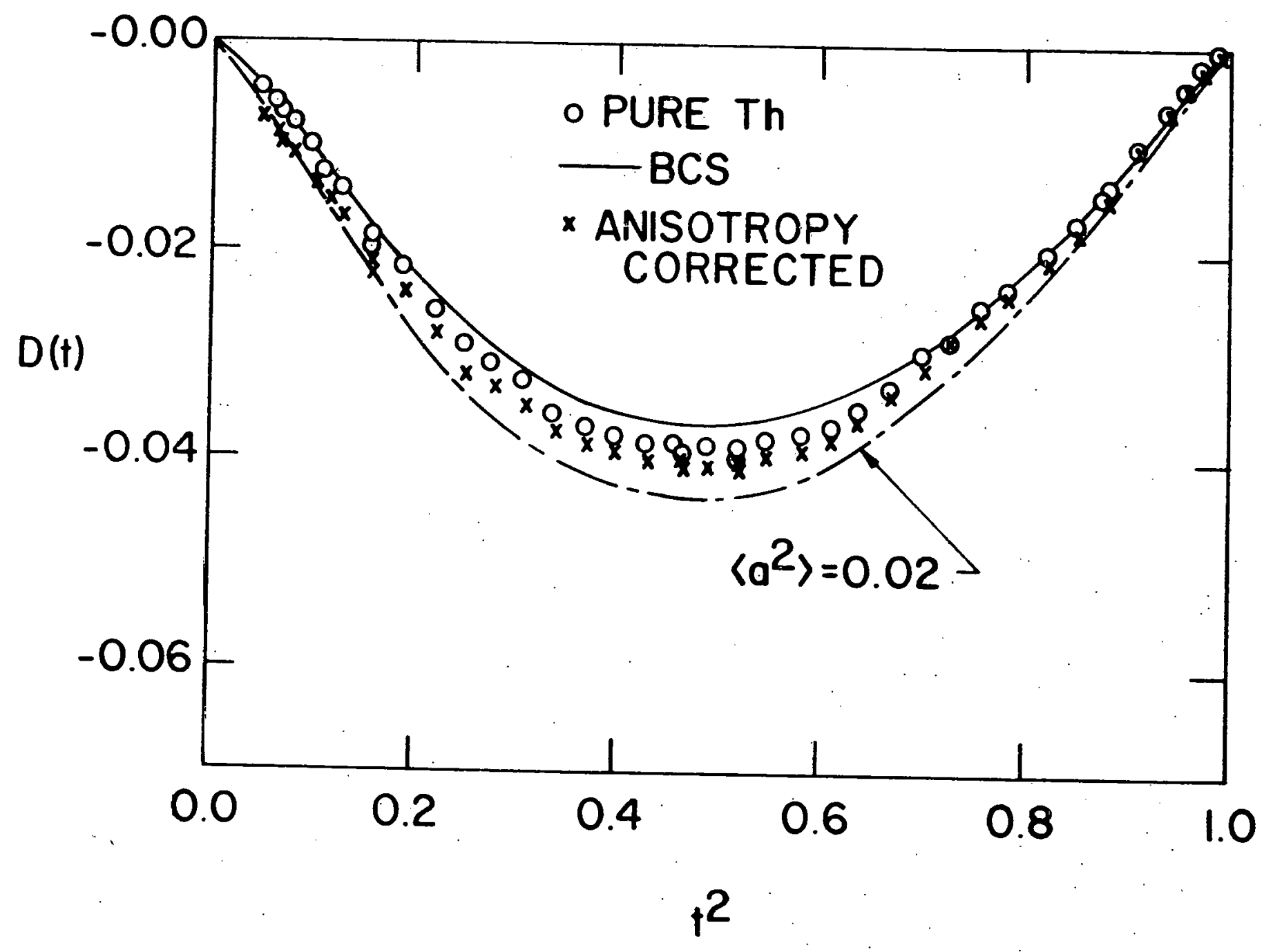


where the superscript "O" indicates the BCS value of $\tilde{\mathrm{H}}_{0}^{2}$. Using Equation 54, we may write Equation 67 as

$$
\gamma=\frac{0.168 \mathrm{H}_{\mathrm{O}}^{2} \mathrm{~V}_{\mathrm{M}}}{\mathrm{T}_{\mathrm{C}}^{2}\left[1-2<\mathrm{a}^{2}>\mathrm{X}_{\mathrm{H}}(0, \lambda)\right]} .
$$

Substitution of $\mathrm{H}_{\mathrm{O}}=159.73 \mathrm{Oe}, \mathrm{V}_{\mathrm{M}}=19.7 \mathrm{~cm}^{3} / \mathrm{mole}$, $\mathrm{T}_{\mathrm{C}}=1.390 \mathrm{~K},\left\langle\mathrm{a}^{2}\right\rangle=0.02$, and $\mathrm{x}_{\mathrm{H}}(0,0)=1$ into Equation 68 gives $Y=4.55 \mathrm{~mJ} / \mathrm{mole}-\mathrm{K}^{2}$, a value about $5 \%$ larger than the $4.34 \mathrm{~mJ} / \mathrm{mole}^{2} \mathrm{~K}^{2}$ obtained by $\mathrm{DF}$.

The study of anisotropy corrections to $\mathrm{H}_{0}, \mathrm{D}(\mathrm{t})$, and $\gamma$ for the Thu alloys was limited to four samples: Th-0.030, $-0.059,-0.102$, and -0.127 at.\% U. $\rho_{r}$ values, however, were obtained for all the alloys and are listed in Table 3 along with the measured values of $\delta T_{C}=T_{C}-T_{C p}$, where $T_{C p}$ is taken to be the DF value of $1.390 \mathrm{~K}$. These data are presented in Figures 32 and 33. Also included in Figure 33 are the results of Anderson et al. (131), indicated by the dashed curve, for the depression of $\mathrm{T}_{\mathrm{C}}$ of pure thorium by carbon impurities, which have a small valence effect. It should be noted that the scattering cross section of a uranium impurity is only about $10 \%$ the cross section of a carbon impurity (133) .

In the calculation of anisotropy corrections for the four Thu alloys, the value of $\alpha^{i}=100$ obtained by Anderson (133) was used to determine values of $X$ and hence $\lambda$. The 
Table 3. Residual resistivity ratios and $\delta T_{C}$ values for ThU

\begin{tabular}{ccc}
\hline Sample & $\rho_{r}$ & $\delta T_{C}(\mathrm{~K})$ \\
\hline Th-0.000 at.\% U & 0.034 & -0.030 \\
Th-0.030 at.\% U & 0.044 & -0.208 \\
Th-0.052 at.\% U & 0.053 & -0.377 \\
Th-0.059 at.\% U & 0.055 & -0.376 \\
Th-0.077 at.\% U & 0.057 & -0.527 \\
Th-0.102 at.\% U & 0.063 & -0.665 \\
Th-0.127 at.\% U & 0.068 & -0.732 \\
Th-0.150 at.\% U & 0.076 & -0.896 \\
Th-0.161 at.\% U & 0.077 & -0.903 \\
Th-0.215 at.\% U & 0.102 & -1.086 \\
\hline
\end{tabular}

value of $\lambda$ for $\underline{T h}-0.102$ at.\% $U$ was 1.003 , so $X_{H}(\lambda, t)$ values were obtained from the $x_{H}(1, t)$ plot given in Figure 1 of Clem II. The values of $\lambda$ obtained for Th-0.030, -0.059, and 0.127 at. $\% U$ were $0.700,0.875$, and 1.082 , respectively, and $x_{H}(t, \lambda)$ functions had to be derived for each of these samples. For $\lambda \neq 0, x_{H}(t, \lambda)$ is defined in terms of another function $\tilde{\mathrm{x}}_{\mathrm{H}}(t, \lambda)$, where

$$
\mathrm{x}_{\mathrm{H}}(t, \lambda)=\left[\frac{\varepsilon_{0}(t)}{\varepsilon_{0}(0)} \frac{\mathrm{H}_{C}(0)}{\mathrm{H}_{C}(t)}\right]^{2} \tilde{\mathrm{x}}_{\mathrm{H}}(t, \lambda) .
$$

Clem II shows that the function $\tilde{\mathrm{x}}_{\mathrm{H}}(t, \lambda)$ can be expanded 


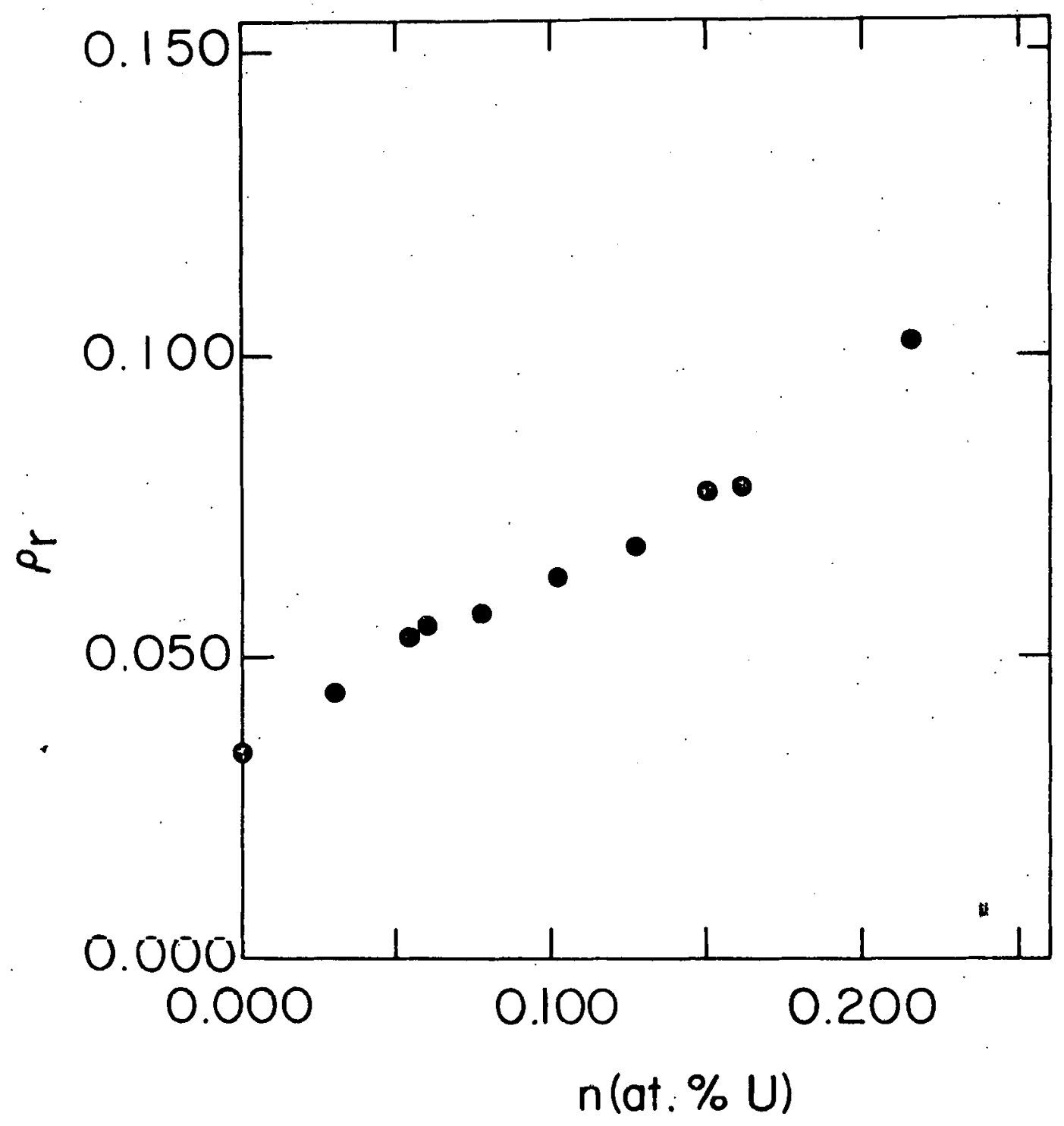

Figure 32. Residual resistivity ratio, $\rho_{r^{\prime}}$ as a function of uranium concentration for ThU? 


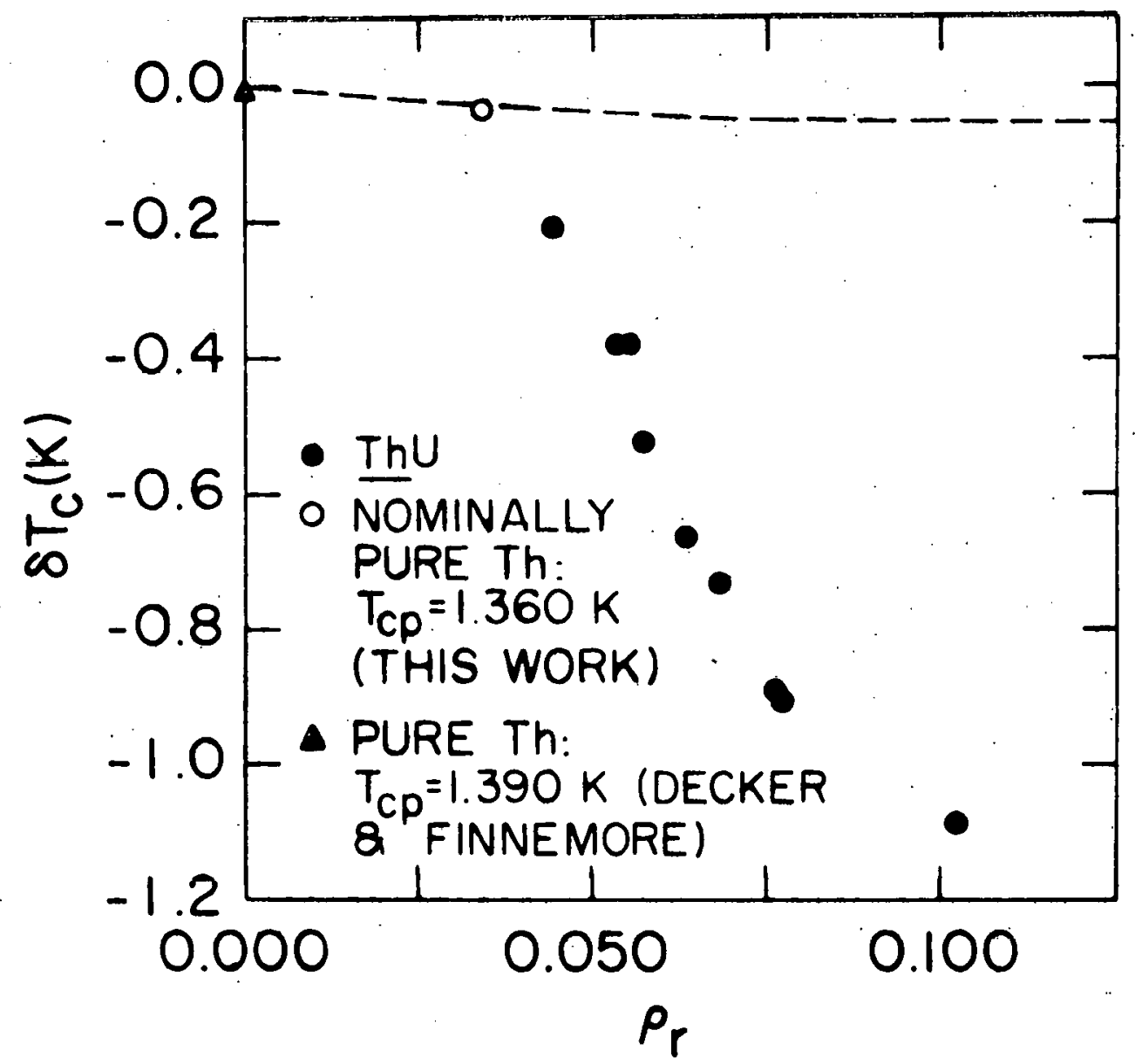

Figure 33. Depression of $T_{C}$ for ThU as a function of $\rho_{r}$. The pure Th sample of Decker and Finnemore (72), indicated by the solid triangle, is taken as the reference $\mathrm{T}_{\mathrm{cp}}$ value. The dashed curve, from the work of Anderson et al. (131), indicates the depression of $T_{C}$ which would be obtained for $a$ simple nonmagnetic impurity with a small valence effect. 
about the critical temperature in terms of $(1:-t)$,

$$
\tilde{x}_{H}(t, \lambda)=B_{1}(\lambda)(1-t)+B_{2}(\lambda)(1-t)^{2}+\cdots,
$$

where $B_{1}(\lambda)$ and $B_{2}(\lambda)$ are given by Equations 34 and 35 in Clem II. The zero-temperature result is given by

$$
\tilde{x}_{H}(0, \lambda)=\left\{\begin{array}{l}
\pi / 2 y-\left(1-y^{2}\right)^{1 / 2} \cos ^{-1} y / y-x(\lambda), y \leq 1 \\
\pi / 2 y+\left(y^{2}-1\right)^{1 / 2} \cosh ^{-1} y / y-x(\lambda), y \geq 1
\end{array}\right\},
$$

where $y=\gamma_{e} \lambda$ and $x(\lambda)$ is defined by

$$
\chi(\lambda) \equiv \psi\left(\frac{1+\lambda}{2}\right)-\psi(1 / 2) \quad .
$$

Clem II shows that Equations 70 and 71 are sufficient to give a fairly good approximation to $\tilde{x}_{H}(t, \lambda)$ versus $t^{2}$ as a function of $\lambda$. We have calculated $\tilde{x}_{H}(t, \lambda)$ for $\lambda=0.700$, 0.875 , and 1.082 and the results are shown in Figures 34-36. The horizontal dashed line in each figure is the $\tilde{\mathrm{x}}_{\mathrm{H}}(0, \lambda)$ value obtained from Equation 71 , and the dashed curve is the result of the power series expansion given by Equation 70. The solid curves are interpolated by eye between the two dashed curves and the accuracy of this method should be approximately $2 \%$, as estimated in Clem II. Using values of $\tilde{\mathrm{x}}_{H}(t, \lambda)$ and Equation 69, the function $x_{H}(t, \lambda)$ was calculated and the 


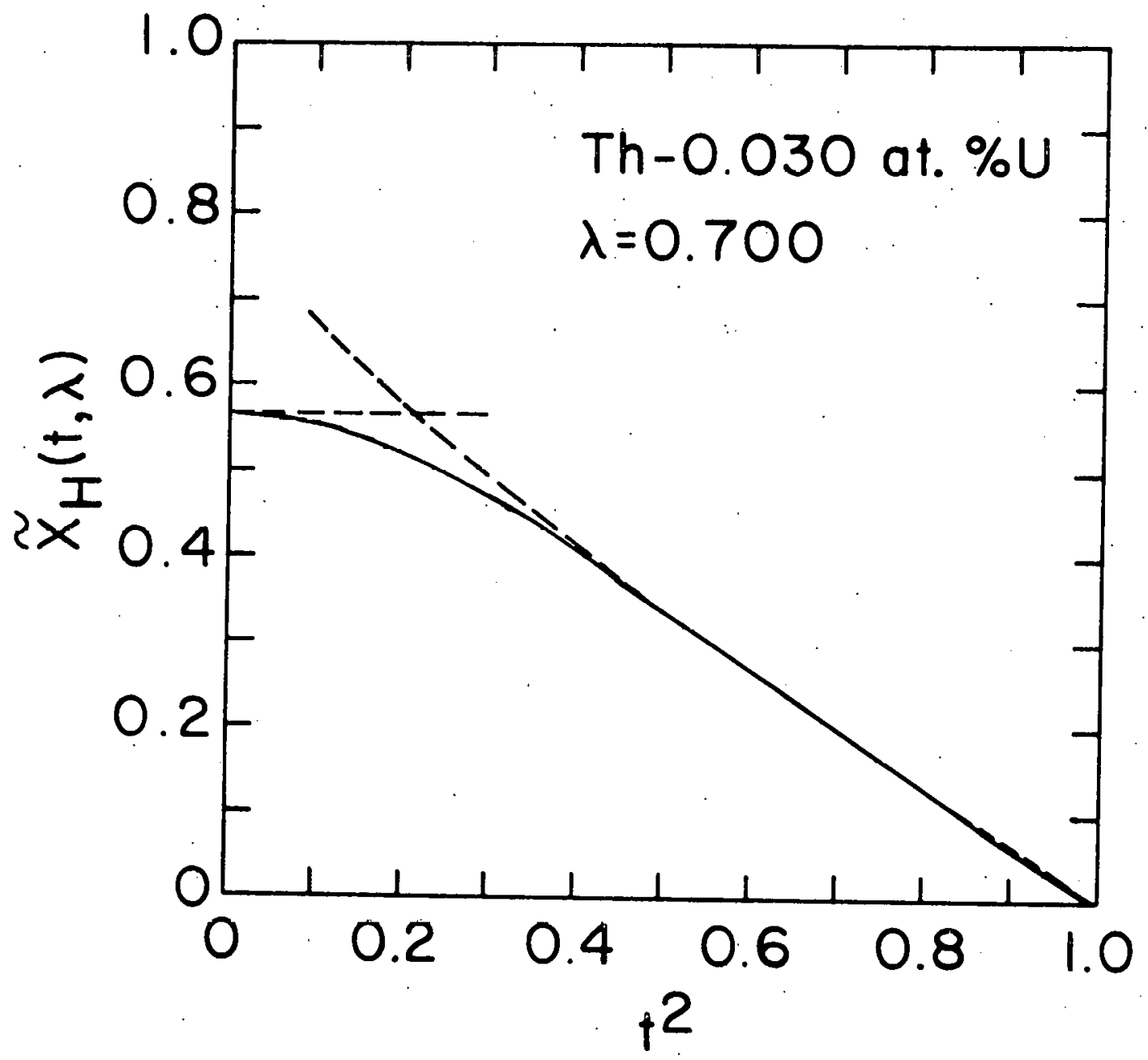

Figure 34. $\tilde{x}_{H}(t, \lambda)$ versus $t^{2}$ for Th-0.030 at.\% $U$ with $\lambda=0.700$. The horizontal dashed line is obtained from Equation 71, and the dashed curve is obtained from Equation 70. The solid curve is the result of interpolating by eye between the dashed curves. 


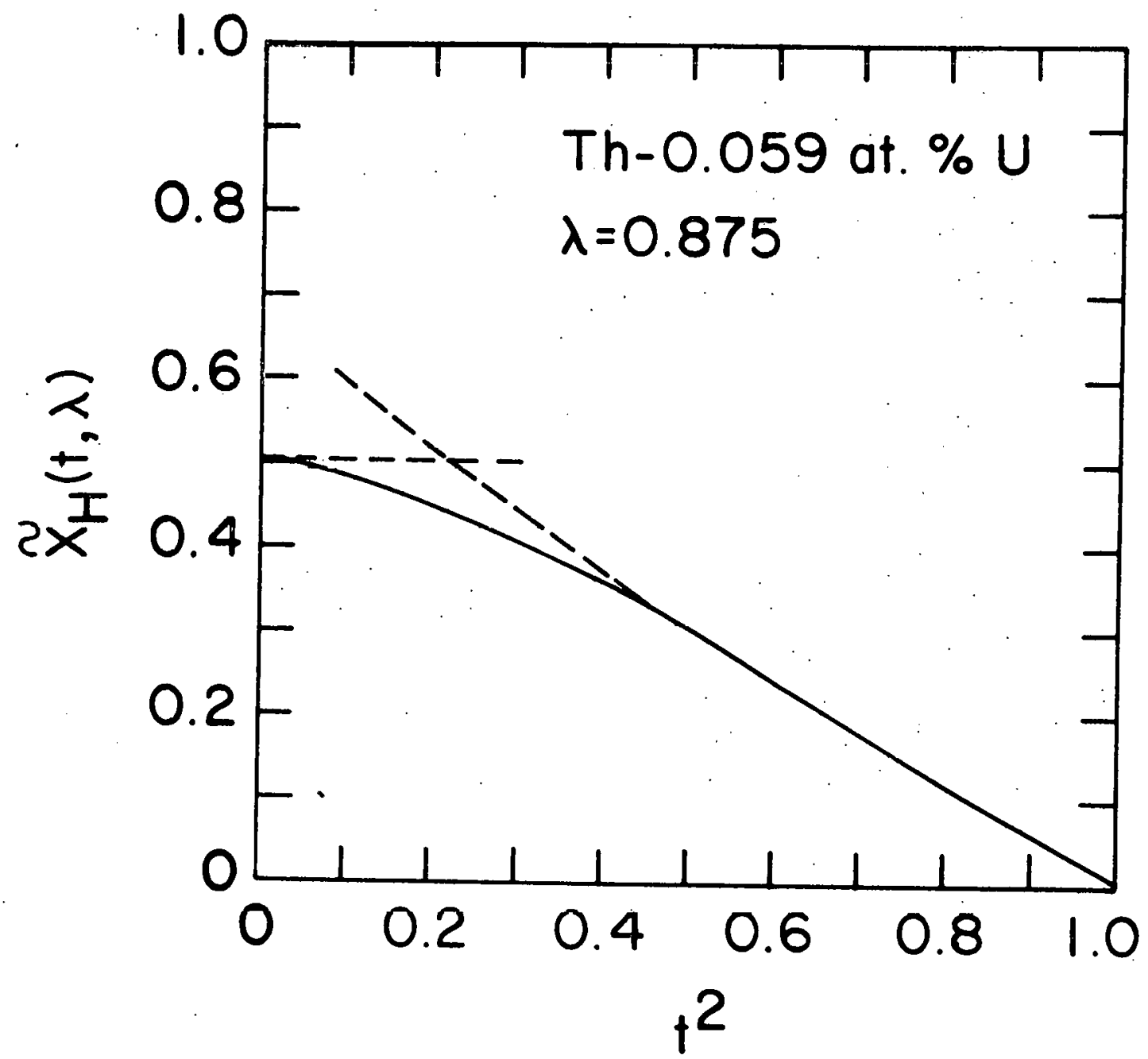

Figure 35. $\tilde{x}_{H}(t, \lambda)$ versus $t^{2}$ for Th-0.059 at.\% $U$ with $\lambda=$ 0.875 . The horizontal dashed line is obtained from Equation 71, and the dashed curve is obtained from Equation 70. The solid curve is the

- result of interpolating by eye between the dashed curves. 


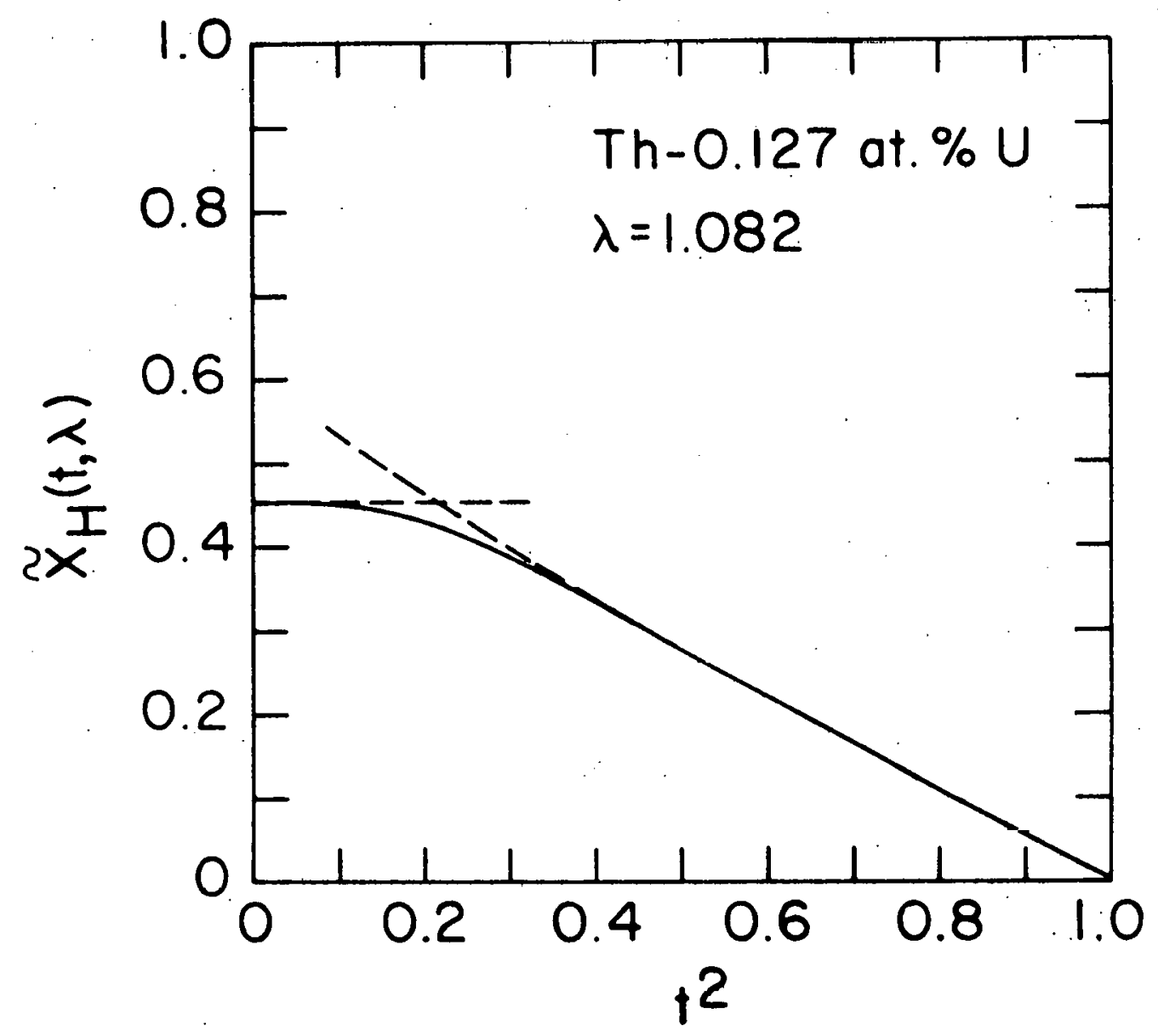

Figure 36. $\tilde{x}_{H}(t, \lambda)$ versus $t^{2}$ for Th-0.127 at.\% $U$ with $\lambda=$ 1.082. The horizontal dashed line is obtained from Equation 71, and the dashed curve is obtained from Equation 70. The solid curve is the result of interpolating by eye between the dashed curves. 
results are shown in Figures 37-39.

An anisotropy corrected $\mathrm{H}_{\mathrm{O}}$ was obtained for each alloy using the $x_{H}(t, \lambda)$ versus $t$ plots and Equation 65. The results of the calculations are given in Appendix C. The anisotropy corrected $\mathrm{H}_{0}$ values were then used to calculate the deviation functions for the alloys, which are also listed in Appendix $C$ and shown in Figures 40-43. The critical field curve data for these ThU alloys deviate a maximum of about $0.6 \%$ from the BCS theory. The anisotropy corrected $\mathrm{H}_{0}$ values and Equation 68 were used to calculate $\gamma$ values for the alloys. The results of these calculations are listed in Table 4 .

Table 4. Calculated anisotropy corrected values of pure Th and Thu

\begin{tabular}{lccc}
\hline \multicolumn{1}{c}{ Sample } & $\lambda$ & $\mathrm{H}_{\mathrm{O}}(\mathrm{Oe})$ & $\gamma\left(\mathrm{mJ} / \mathrm{mole}^{2}\right)$ \\
\hline Pure Th(DF) & $\sim \mathrm{K}$ & 159.73 & 4.55 \\
Th-0.030 at.\% U & 0.700 & $137.16 \pm 1.46$ & $4.57 \pm 0.09$ \\
Th-0.059 at.\% U & 0.875 & $117.39 \pm 0.57$ & $4.54 \pm 0.04$ \\
Th-0.102 at.\% U & 1.003 & $84.97 \pm 0.21$ & $4.64 \pm 0.03$ \\
Th-0.127 at.\% U & 1.082 & $76.97 \pm 0.41$ & $4.62 \pm 0.05$ \\
\hline
\end{tabular}




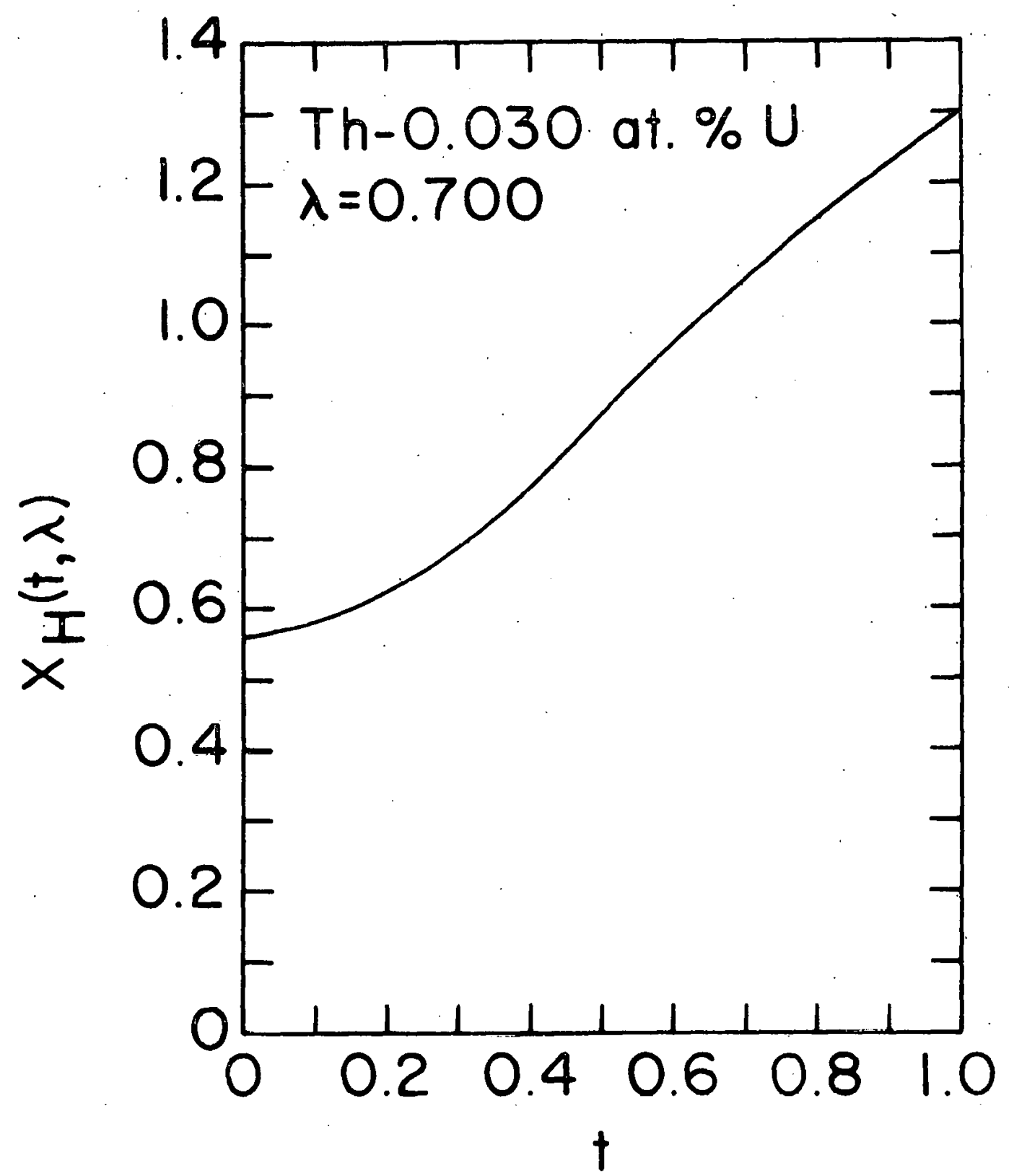

Figure 37. $x_{\mathrm{H}}(t, \lambda)$, which describes the effectiveness of anisotropy corrections to $\mathrm{H}_{\mathrm{c}}^{2} / 8 \pi \gamma^{\mathrm{T}} \mathrm{C}^{2}$, versús $t$ for $T h-0.030$ at. $\%$ with $\lambda \stackrel{c}{=} 0.700$. 


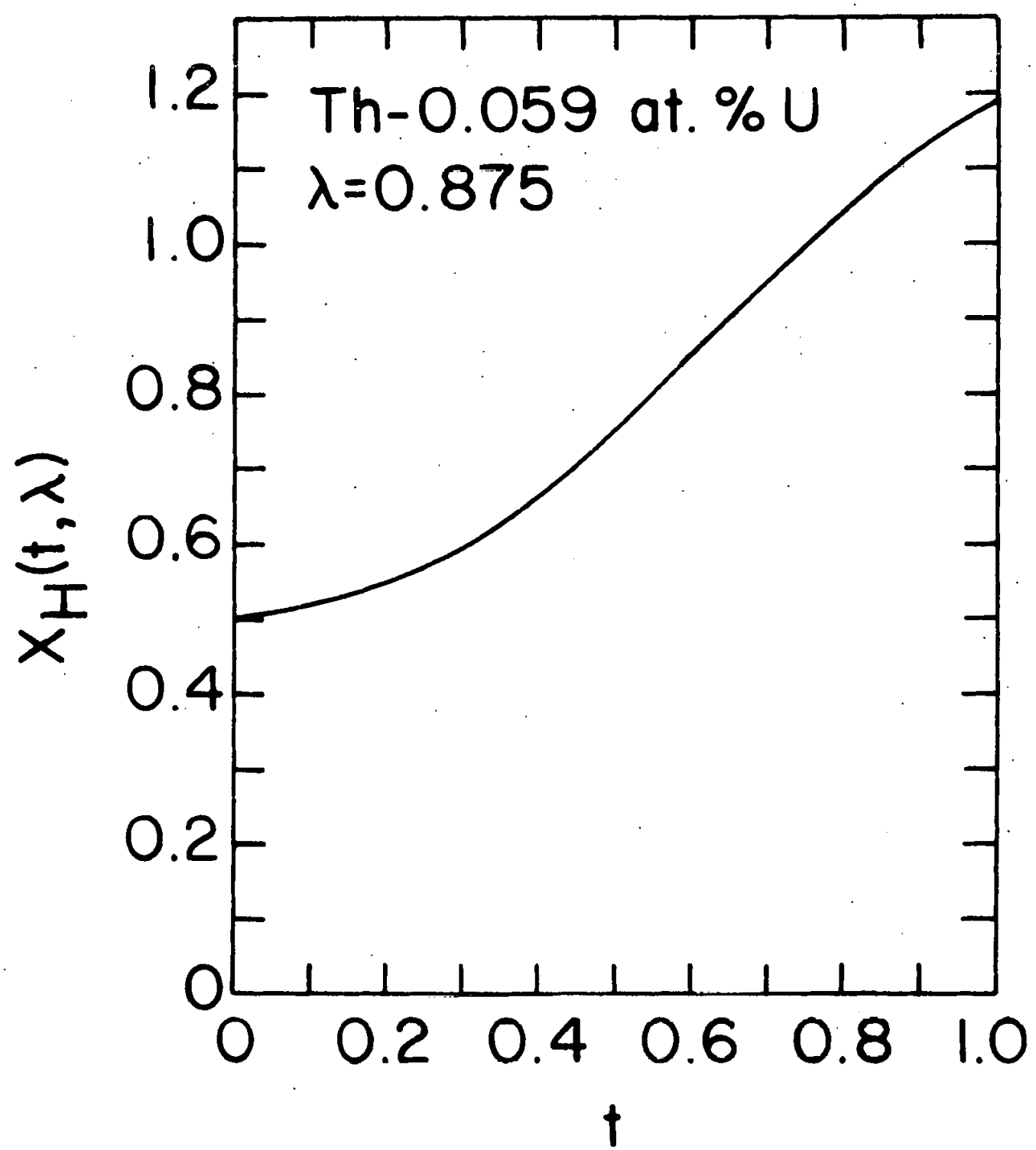

Figure 38. $x_{H}(t, \lambda)$, which describes the effectiveness of anisotropy corrections to $\mathrm{H}_{C}^{2} / 8 \pi \gamma^{\mathrm{T}} \mathrm{T}_{\mathrm{C}}^{2}$, versus $t$ for $\mathrm{Th}-0.059$ at. $\% \mathrm{U}$ with $\lambda=0.875$. 


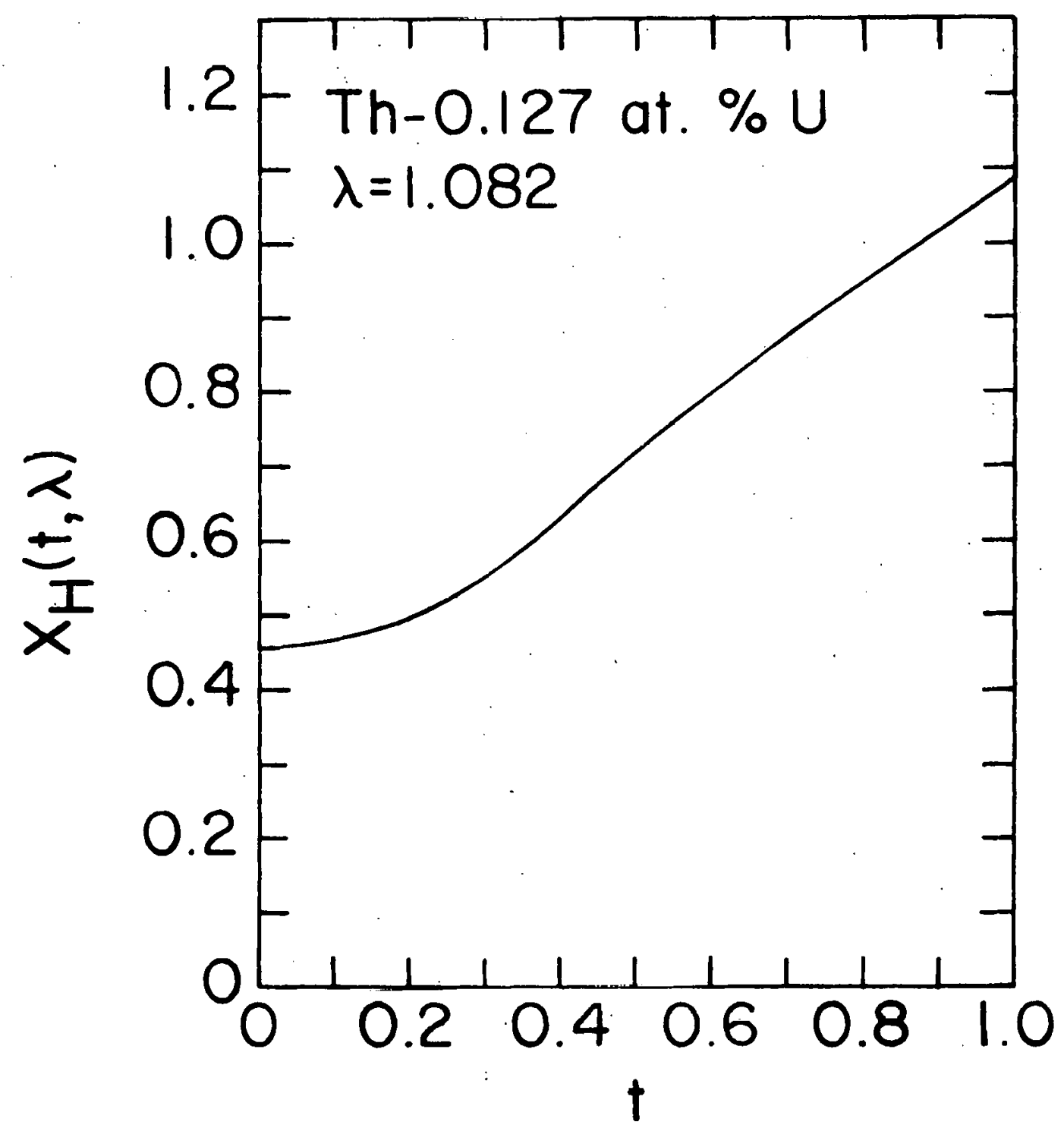

Figure 39. $x_{H}(t, \lambda)$, which describes the effectiveness of anisotropy corrections to $\mathrm{H}_{\mathrm{C}}^{2} / 8 \pi \gamma^{\mathrm{T}} \mathrm{T}_{\mathrm{C}}^{2}$, versus $t$ for Th-0.127 at. $\% \mathrm{U}$ with $\lambda=1.082$. 


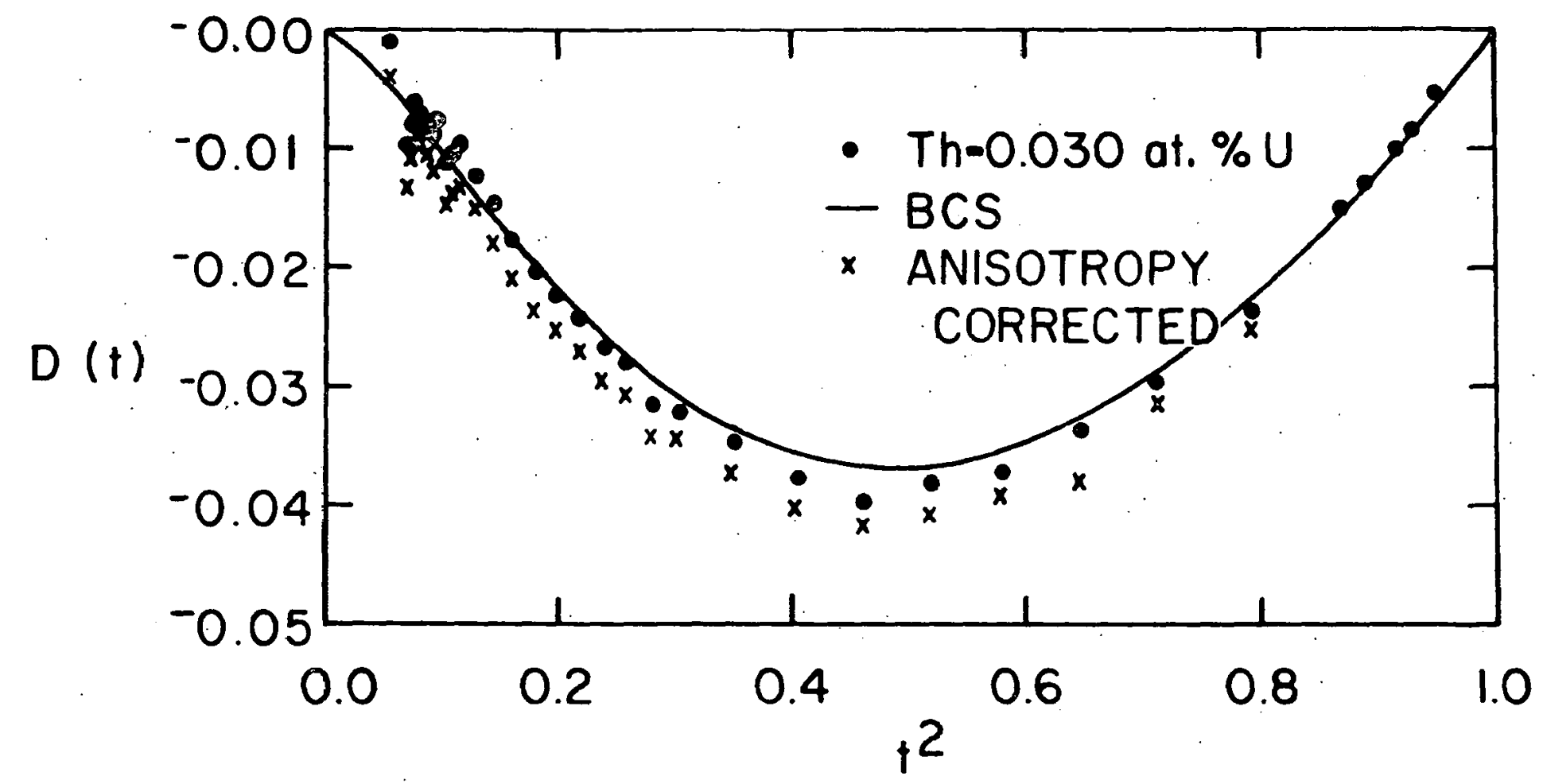

Figure 40. Deviation function for Th-0.030 at.\% $U$ using both the simple BCS theory $\mathrm{H}_{0}$ (solid circles) and the anisotropy corrected $\mathrm{H}_{0}\left(\mathrm{x}^{\prime} s\right)$ compared to the BCS theory (solid curve). 


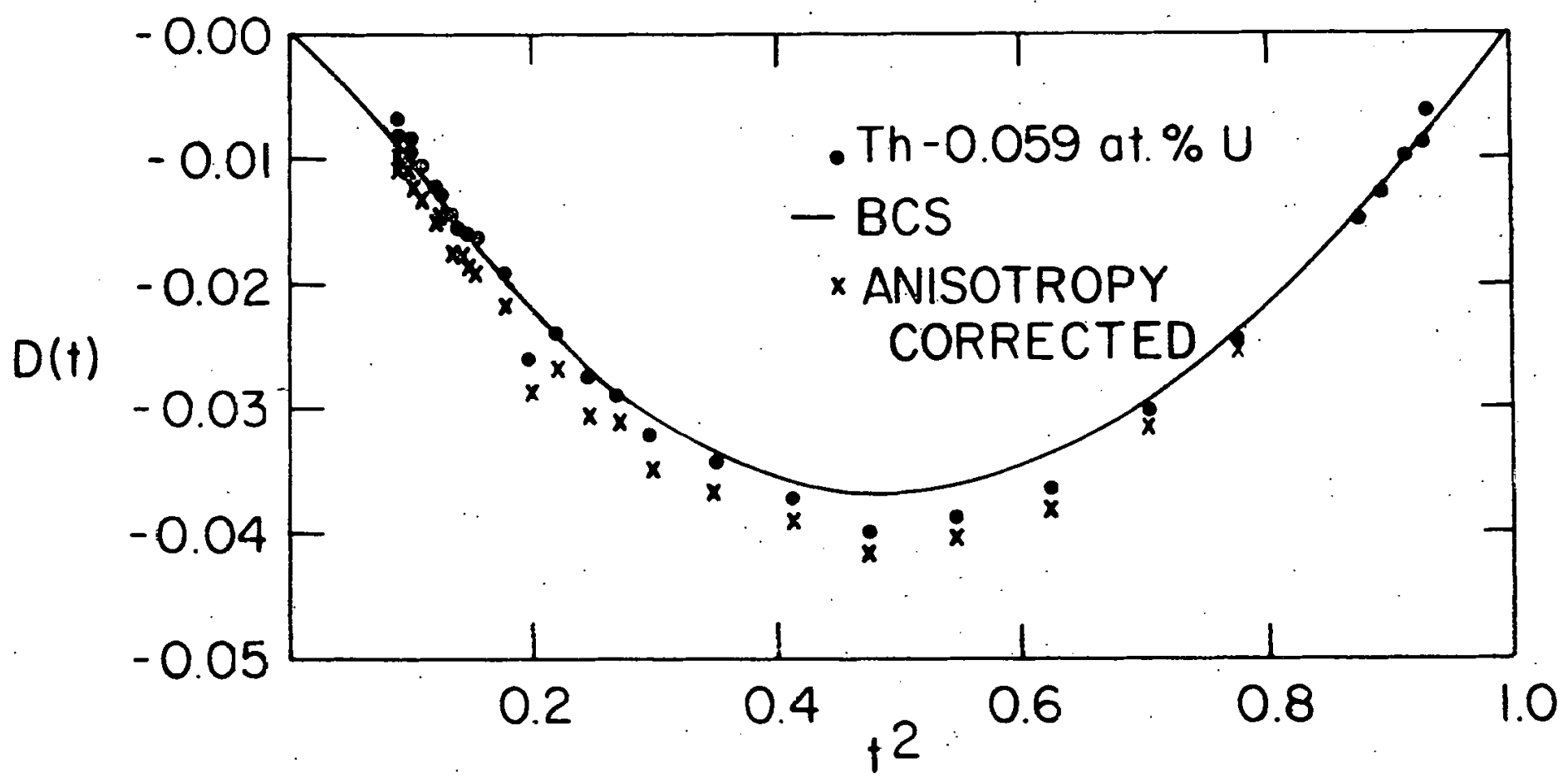

Figure 41. Deviation function for Th-0.059 at.\% U using both the simple BCS theory $\mathrm{H}_{0}$ (solid circles) and the arisotropy corrected $\mathrm{H}_{0}$ ( $x^{\prime} s$ ) compared to the BCS theory (solid curve). 


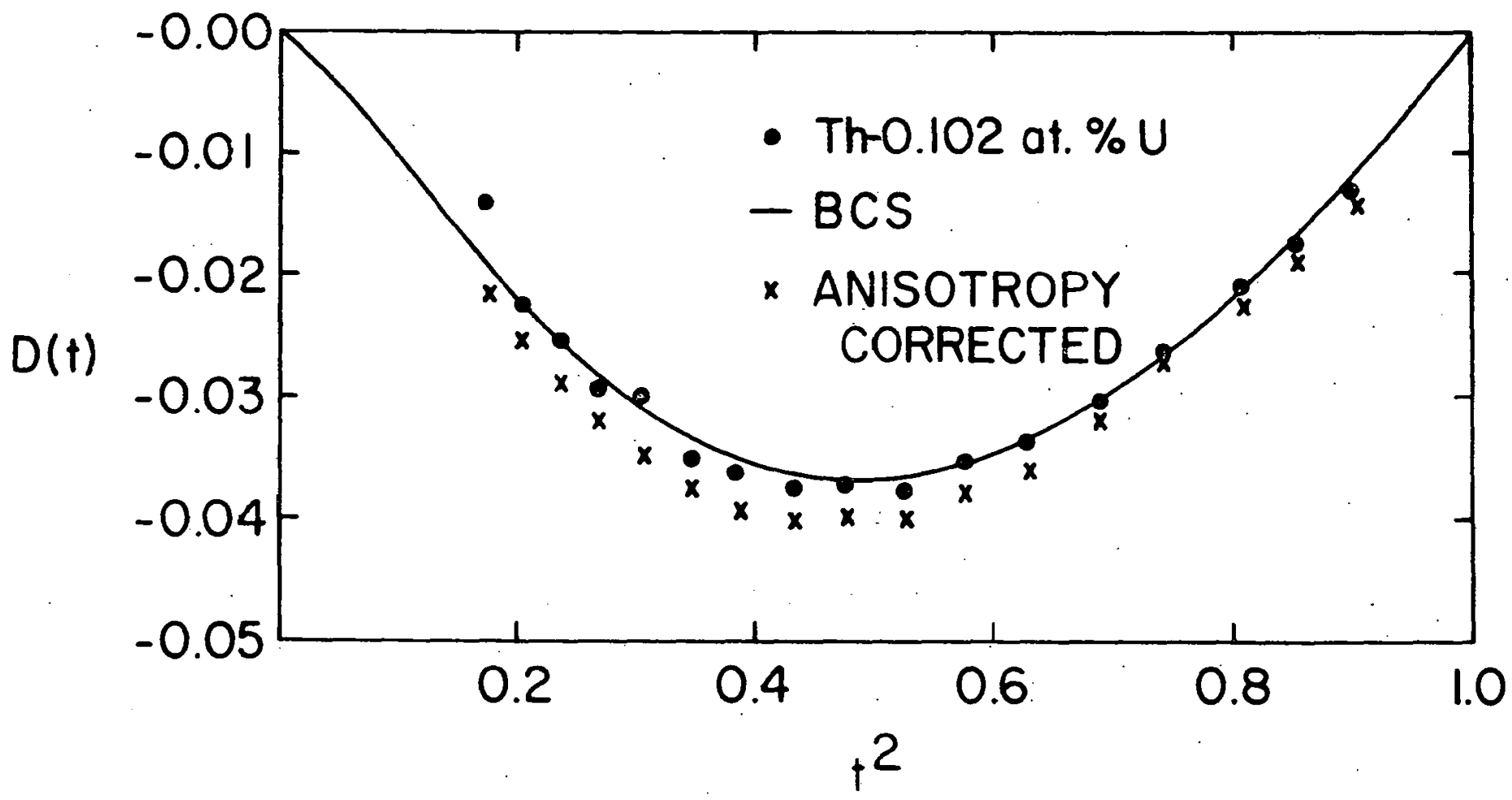

Figure 42. Deviation function for Th-0.102 at. $\%$ U using both the simple BCS theory $\mathrm{H}_{0}$ (solid circles) and the anisotropy corrected $\mathrm{H}_{0}$. $\left(x^{\prime} s\right)$ compared to the BCS theory (solid curve). 


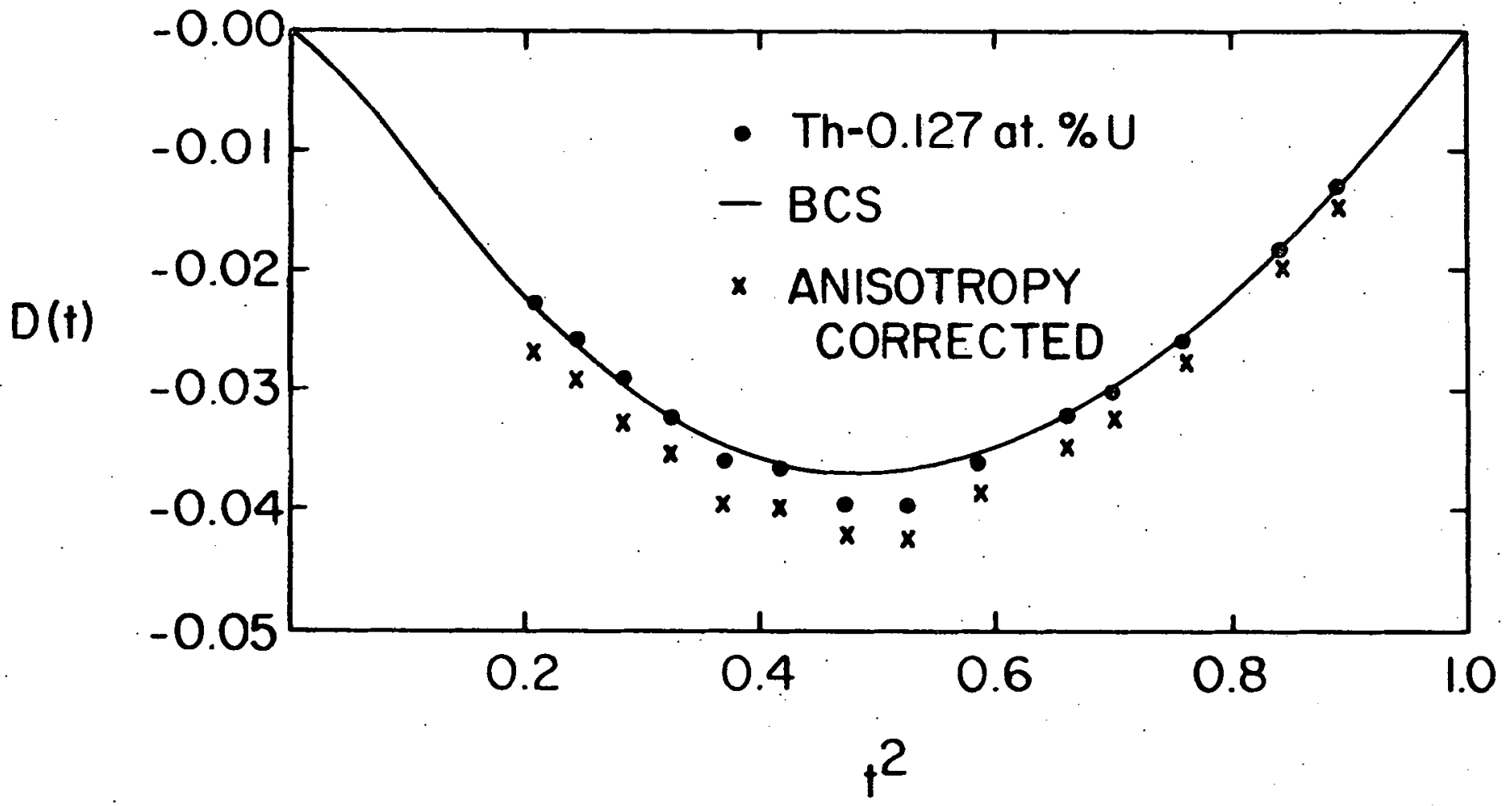

Figure 43. Deviation function for Th-0.127 at.\% U using both the simple $\mathrm{BCS}$ theory $\mathrm{H}_{\mathrm{O}}$ (solid circles) and the anisotropy corrected $\mathrm{H}_{0}$ ( $\left.x^{\prime} s\right)$ compared to the BCS theory (solid curve). 
An examination of the anisotropy corrected $\gamma$ values listed in Table 4 indicates that within the scatter of the data, the value of $\gamma$ remains essentially constant. The $\mathrm{H}_{\mathrm{C}}(\mathrm{T})$ measurements need to be extended to lower temperatures than were reached in this experiment to obtain experimental values for $\mathrm{H}_{0}$ and $Y$ using Equation 17 before any definite statement can be made concerning a $\gamma$ enhancement. Alternatively, a specific heat study of these samples would be useful because, as discussed above, the ThU alloys used by Luengo et al. (113) are of questionable homogeneity. 


\section{SUMMARY}

This research shows that the critical field curves for Thu are in agreement with the BCS theory critical field curves to an accuracy of $1 \%$, and thus provides additional experimental evidence that the rapid depression of the superconducting transition temperature of pure thorium by the uranium impurities is due to pair-weakening rather than to pair-breaking mechanisms. The effects of spin-flip scattering, if present at all, are at least an order of magnitude smaller than the pair-weakening effects. Additional experimental evidenceis provided by the fact that the reduced specific heat jumps at $\mathrm{T}_{\mathrm{C}}$ for these alloys, obtained from the slope of the critical field at $T_{C}$ and Rutger's formula (Equation 15), are in close agreement with the predictions of the BCS theory.

Using a BCS model for ThU, one would expect, from Equation 19, that the reduction in $\mathrm{T}_{\mathrm{C}}$ would enter through $\mathrm{N}(0) \mathrm{V}^{\prime}$. However, within the accuracy of the data, the specific heat coefficient, $Y$, and hence, $N(0)$, remains essentially constant. This implies that the major effect is due to a reduction in the BCS effective electron-electron interaction, $\mathrm{V}^{\prime}$. The Kaiser model (96) would attribute the reduction of $V^{\prime}$ to resonance scattering at the uranium sites, which increases the effective coulomb repulsion between the mates 
of the cooper pairs. The Riblet model (100), on the other hand, would say that the cooper pairing interaction is reduced because electron-localized spin fluctuation coupling occurs. Other complex interactions may play a role. Although no one specific mechanism can be identified as being responsible for the destruction of superconductivity in ThU, this research strongly indicates that the primary interactions are time-reversal invariant, and hence, pairweakening rather than pair-breaking: 


\section{BIBLIOGRAPHY}

1. H. K. Onnes, Commun. Kamerlingh Onnes Lab. Univ. Leiden, Suppl. No. 34b, 55 (1913).

2. J. Bardeen and J. R. Schrieffer, in progress in Low Temperature Physics, edited by C. J. Gorter (NorthHolland Publishing Company, Amsterdam, 1961), Vol. III, p. 170 .

3. M. Tinkham, in Low-Temperature Physics, edited by C. de Witt, B. Dreyfus, and P. G. de Gennes (Gordon and Breach, Science Publishers, Inc., New York, 1962), p. 149 .

4. J. M. Blatt, Theory of Superconductivity (Academic Press, New York, 1964).

5. J. R. Schrieffer, Theory of Superconductivity (W. A. Benjamin, Inc., New York, I964).

6. G. R. Rickayzen, Theory of Superconductivity (Interscience Publishers, New York, 1965).

7. D. Shoenberg, Superconductivity (Cambridge University Press, Cambridge, England, 1965), 2nd. ed.

8. P. G. de Gennes, Superconductivity of Metals and Alloys (W. A. Benjamin, Inc., New York, I966).

9. E. A. Lynton, Superconductivity (Metheun \& Co., Ltd., London, 1969), 3rd. ed.

10. C. G. Kuper, Introduction to the Theory of Superconductivity (clarendon Press, oxford, England, 1968).

11. R. D. Parks, ed., Superconductivity (Gordon and Breach, Science Publishers, Inc., New York, 1969), Vol. I and 2.

12. R. P. Wallace, ed., Superconductivity (Gordon and Breach, Science Publishers, Inc., New York, 1969), Vol. 1 and 2 .

13. D. J. Quinn III and W. B. Ittner III, J. Appl. Phys. 33, 748 (1962).

14. G. T. Meaden, Electrical Resistance of Metals (Plenum Press, New York, 1965), pp. 15-18. 
15. W. H. Keesom and J. A. Kok, Commun. Kamerlingh Onnes Lab. Univ. Leiden, No. 22le (1932).

16. P. Ehrenfest, Commun. Kanerlingh Onnes Lab. Univ. Leiden, No. 75b (1933).

17. W. H. Keesom, Rapp et. Disc. 4e Congr. Phys. Solvay; 228 (1924).

18. A. J. Rutgers, Physica I, 1055 (1934).

19. C. J. Gorter, Arch. Mus. Teyler I, 378 (1933).

20. W. Meissner and R. Ochsenfeld, Naturwiss. $\underline{21}, 787$ (1933).

21. C. J. Gorter and H. B. G. Casimir, Physica 1, 306 (1934).

22. C. J. Gorter and H. B. G. Casimir, Phys. Z. 35,963 (1934); Z. Tech. Phys. 15, 539 (1934).

23. F. London and H. London, Proc. Roy. Soc. (London) Al49, 71 (1935); Physica 2., 341 (1935).

24. D. Shoenberg, Proc. Roy. Soc. (London) Al75, 49 (1940).

25. A. B. Pippard, Proc. Roy. Soc. (London) A216, 547 (1953).

26. H. Fröhlich, Phys. Rev. 79, 845 (1950).

27. E. Maxwell, Phys. Rev. 78, 477 (1950); C. A. Reynolds, B. Serin, W. H. Wright, and L. B. Nesbitt, Phys. Rev. 78, 487 (1950).

28. B. B. Goodman, Proc. Phys. Soc. (London) $\underline{A 66}, 216$ (1953).

29. W. S. Corak, B. B. Goodman, C. B. Satterthwaite, and A. Wexler, Phys. Rev. 96, 1442 (1954); Phys. Rev. 102, 656 (1956); W. Corak and C. B. Satterthwaite, Phys. Rev. 102, 662 (1956).

30. G. S. Blevins, W. Gordy, and W. M. Fairbank, Phys. Rev. 140, 1215 (1955); R. E. Glover and M. Tinkham, Phys. Rev. 104, 844 (1956).

31. L. N. Cooper, Phys. Rev. 104, 1189 (1956). 
32. J. Bardeen, L. N. Cooper, and J. R. Schrieffer, Phys. Rev. 108, 1175 (1957).

33. D. K. Finnemore and D. E. Mapother, Phys. Rev. 140, A507 (1965).

34. R. Meservey and B. B. Schwartz, in Superconductivity, edited by R. D. Parks, (Gordon and Breach Science Publishers, Inc., New York, 1969), Vol. 1, p. 120.

35. B. Mühlschlegel, Z. Physik 155, 313 (1959).

36. D. L. Decker, D. E. Mapother, and R. W. Shaw, Phys. Rev. 112,1888 (1958).

37. E. P. Harris and D. E. Mapother, Phys. Rev. 165, 522 (1968).

38. J. C. Swihart, D. J. Scalapino, and Y. Wada, Phys. Rev. Letters 14, 106 (1965).

39. W. L. MoMillan, Phys. Rev. 167, 331 (1968).

40. J. R. Clem, Ann. Phys. 40, 268 (1966); Phys. Rev. 153, 449 (1967).

41. G. J. van den Berg, in Progress in Low. Temperature Physics, edited by C. J. Gorter (North-Holland PubIishing Company, Amsterdam, 1964), Vol. IV, p. 194.

42. M. D. Daybell and W. A. Steyert, Rev. Mod. Phys. $\underline{40}$, 380 (1968).

43. A. J. Heeger, in Solid State Physics, edited by F. Seitz, D. Turnbuli, and H. Ehrenreich (Academic Press, New York, 1969), Vol, 23, p. 283.

44. J. Kondo, in Solid State Physics, edited by F. Seitz, D. Turnbull, and $\mathrm{H}$. Ehrenreich (Academic Press, New York, 1969), Vol. 23, p. 183.

45. K. H. Bennemann and J. W. Garland, Intern. J. Magnetism I. 97 (197.1).

46. M. B. Maple, in AIP Conference Proceedings, Superconductivity in d- and f- Band Metals, edited by D. H. Douglass (AIP, New York, 1972), No. 4, p. 175.

47. M. B. Maple, in Magnetism, edited by H. Suhl (Academic Press, New York), Vol. V (to be published). 
48. E. Müller-Hartmann, in Magnetism, edited by H. Suhl (Academic Press, New York), Vol. V (to be published).

49. R. D. Parks, in Superconductivity, edited by R. P. Wallace (Gordon and Breach Science Publishers, Inc., New York, 1969), Vol. 2, p. 623.

50. K. H. Bennemann, in Superconductivity, edited by R. P. Wallace (Gordon and Breach Science Püblishers, Inc., New York, 1969), Vol. 1, p. 1 .

51. K. Maki, in Superconductivity, edited by R. D. Parks (Gordon and Breach Science Publishers, Inc., New York, 1969), Vol. 2, p. 1035.

52. J. Friedel, Can. J. Phys. 34, 1190 (1956); Nuovo Cimento Suppl. 7,289 (1958).

53. P. W. Anderson, Phys. Rev. 124, 41 (1961).

54. J. Kondo, Progr. Theoret. Phys. (Kyoto) 32, 37 (1964).

55. P. W. Anderson and A. M. Clogston, Bull. Am. Phys. Soc. $\underline{6}, 124$ (1961).

56. J. R. Schrieffer and P. A. Wolff, Phys. Rev. 149, 491 (1966).

57. H. Suhl, Phys. Rev. Letters 19, 442 (1967); M. J. Levirie and H. SuhI, Phys. Rev: 171, 567 (1968); M. J. Levine, T. V. Ramakrishnan, and R. A. Weiner, Phys. Rev. Letters 20, 1370 (1968); N. Rivier and M. J. Zuckermann, phys. Rev. Letters 2l, 904 (1968); N. Rivier, M. Sunjic, and M. J. Zuckermann, Phys. Letters 28A, 492 (1969).

58. A. D. Caplin and C. Rizzuto, Phys. Rev. Letters 21, 746 (1968).

59. E. A. Lynton, B. Serin, and M. Zucker, J. Phys. Chem. Solids $\underline{3}, 165$ (1957).

60. G. Chanin, E. A. Lynton, and B. Serin, Phys. Rev. 114, 719 (1959).

61. W. Buckel and R. Hilsch, Z. Phys. 128, 324 (1950).

62. B. T. Matthias, H. Suhl, and E. Corenzwit, Phys. Rev. Letters 1,92 (1958). 
63. P. W. Anderson, J. Phys. Chem. Solids 11, 26 (1959).

64. A. A. Abrikosov and L. P. Gor'kov, Zh. Eksp. Teor. Fiz. 39, 1781 (1960) [Sov. Phys. JETP 12, 1243 (1961)].

65. P. G. de Gennes, J. Phys. Radium 23, 510 (1962).

66. S. Skalski, O. Betbeder-Matibet, and P. R. Weiss, Phys. Rev. 136, Al500 (1964).

67. V. Ambegaokar and A. Griffin, Phys. Rev. 137, Al151 (1965).

68. M. A. Wolff and F. Reif, Phys. Rev. 137, A557 (1965).

69. D. K. Finnemore, D. L. Johnson, J. E. Ostenson, F. H. Spedding, and B. J. Beaudry, Phys. Rev. 137, A550 (1965).

70. J. Millstein and M. Tinkham, Phys. Rev. 158, 325 (1967).

71. W. R. Decker, D. T. Peterson, and D. K. Finnemore, Phys. Rev. Letters 18, 899 (1967).

72. W. R. Decker and D. K. Finnemore, Phys. Rev. 172, 430 (1968).

73. M. B. Maple, Phys. Letters 26A, 513 (1968).

74. R. L. Cappelletti and D. K. Finnemore, Phys. Rev. 188, 723 (1969).

75. G. J. Dick and F. Reif, Phys. Rev. 181, 774 (1969).

76. R. A. Hein, R. L. Falge, Jr., B. T. Mattias, and E. Corenzwit, Phys. Rev. Letters 2, 500 (1959).

77. J. E. Crow and R. D. Parks, Phys. Letters 21, 378 (1966).

78. K. H. Bennemann, Phys. Rev, Letters 17, 438 (1966).

79. P. Fulde and K. Maki, Phys. Rev. 141, 275 (1966).

80. K. H. Bennemann, J. W. Garl and, and F. M. Mueller, Pliys. Rev. Letters 23. 169 (1969).

81. E. Müller-Hartmann and J. Zittartz, Phys. Rev. Letters 26, 428 (1971). 
82. A. Ludwig and M. J. Zuckermann, J. Phys. F1, 516 (1971).

83. G. Riblet and K. Winzer, Solid State Commun. 9, 1663 (1971).

84. M. B. Maple, W. A. Fertig, A. C. Mota, D. E. DeLong, D. Wohllenben, and R. Fitzgerald, Solid state Commun. 11. 829 (1972).

85. K. Maki, J. Low Temp. Phys. $\underline{6}, 505$ (1972).

86. G. Riblet and $\mathrm{K}$. Winzer, Solid state Commun. 11, 175 (1972).

87. C. A. Luengo, M. B. Maple, and W. A. Fertig, Solid State Commun. 11, 1445 (1972).

88. E. Müller-Hartmann and J. Zittartz, Solid State Commun. 11, 401 (1972).

89. F. W. Smith, J. Low Temp. Phys. 5, 683 (1971).

90. G. Boato, G. Gallinaro, and C. Rizzuto, Phys. Letters $\underline{5}, 20$ (1963); Rev. Mod. Phys. 36, 162 (1964); Phys. Rev. 148353 (1966).

91. M. J. Zuckermann, Phys. Rev. 140, A899 (1965).

92. C. F. Ratto and A. Blandin, Phys. Rev. 156, 513 (1967).

93. K. Takanaka and F. Takano, Progr. Theoret. Phys. (Kyoto) 36. 1080 (1966).

94. R. Aoki and T. Ohtsuka, J. Phys. Soc. Japan 23, 955 (1967).

95. M. Kiwi and M. J. Zuckermann, Phys. Rev. 164, 548 (1967).

96. A. B. Kaiser, J. Phys. C 3,409 (1970).

97. J. R. Schrieffer and D. C. Mattis, Phys. Rev. 140A, 1412 (1965).

98. J. G. IIuleer and M. B. Maple, J. Low Temp. Phys. 3, 537 (1970).

99. J. G. Huber and M. B. Maple, Solid state Commun. $\underline{8}$, 1987 (1970). 
100. G. Morandi, Solid State Commun. $\underline{6}, 561$ (1968).

101. G. Riblet, Phys. Rev. B3 , 91 (1971).

102. P. Lederer and D. L. Mills, Phys. Rev. 165, 837 (1968).

103. W. L. MaMillan, Phys. Rev. 167, 331 (1968).

104. K. H. Bennemann, Phys. Rev. 183 (1969).

105. F. W. Smith, J. Low Temp. Phys. 6, 435 (1972).

106. D. L. Martin, Proc. Phys. Soc. (London) 78, 1489 (1961).

107. N. Y. Rivier and D. E. Maclaughlin, J. Phys Fl, L48 (1971).

108. M. J. Zuckermann, J. Phys. Ç․ 2130 (1970).

109. B. E. Paton and M. J. Zuckermann, J. Phys. Fl, 125 (1971).

110. M. B. Maple, J. G. Huber, B. R. Coles, and A. C. Lawson, J. Low Temp. Phys. 3 , 137 (1970).

111. D. T. Peterson, D. F. Page, R. B. Rump, and D. K. Finnemore, Phys. Rev. 153, 701 (1967).

112. H. Nagasawa, S. Yoshida, and T. Sugawara, Phys. Letters 26A, 561 (1968).

113. C. A. Luengo, J. M. Cotignola, J. G. Sereni, A. R. Sweedler, M. B. Maple, and J. G. Huber, Solid State Commun. 10, 459 (1972); C. A. Luengo, J. M. Cotignola, J. Sereni, A. R. Sweedler, and M. B. Maple (to be published).

114. J. G. Huber and M. B. Maple, J. Phys. Fl, 893 (1971).

115. J. R. Murray, J. Inst. Metals 87, 94 (1958-59).

116. D. T. Peterson, Ames Lab. A.E.C., Ames, Iowa, private communication, 1973.

117. W. R. Decker, Ph.D. thesis, Iowa State University, 1968 (unpublished).

118. F. G. Brickwedde, H. van Dijk, M. Durieux, J. R. Clement, and J. K. Logan, J. Res. Nat. Bur. Stand. 64A, 1 (1960). 
119. L. J. Williams, Ph.D. thesis, Iowa State University, 1969 (unpublished).

120. F. R. Kroeger and W. A. Rhinehart, Rev. Sci. Instrum. 42, 1532 (1971).

121. T. C. Cetas, Ph.D. thesis, Iowa State University, 1970 (unpublished).

122. A. C. Anderson, R. E. Peterson, and J. E. Robichaux, Rev. Sci. Instrum: 41, 528 (1970).

123. P. W. Sparks and C. A. Swenson, Phys. Rev. 163, 779 (1967).

124. E. Maxwell, Rev. Sci. Instrum. 36, 553 (1965).

125. W. A. Rhinehart and I. Mourlam, Jr., Electronics 38 , $88(1965)$.

126. I. Mourlam, Jr. and W. A. Rhinehart, USAEC Report IS-2389, 1970 (unpublished).

127. J. F. Cochran, D. E. Mapother, and R. E. Mould, Phys. Rev. 103, 1657 (1956).

128. C. A. Swenson, USAEC Report IS-399, 1962 (unpublished).

129. J. E. Gordon, H. Montgomery, R. J. Noer, C. R. Pickett, and R. Tobon, Phys. Rev. 152, 432 (1966).

130. D. Markowitz and L. P. Kadanoff, Phys. Rev. 131, 563 (1963).

131. J. W. Anderson, D. T. Peterson, and D. K. Finnemore, Phys. Rev. 179, 472 (1969).

132. D. U. Gubser, Phys. Rev. Bㅌ, 827 (1972).

133. J. W. Anderson, M.S. Thesis, Iowa State University, 1968 (unpublished). 


\section{ACKNOWLEDGMENTS}

The author wishes to thank his advisor, Professor D. K. Finnemore, for the guidance and encouragement given by him during the course of this research and in the preparation of this manuscript.

He would also like to thank Dr. D. T. Peterson and his group for furnishing and preparing the samples, $\mathrm{Dr}$. J. S. Fritz's group for the detailed chemical analysis of the samples, and Dr. J. R. Clem for his assistance with the anisotropy correction calculations.

Special thanks are extended to $\mathrm{Dr}$. Y. N. Lwin of Western Illinois University for his help and encouragement through the years, to Professor G. C. Danielson for the guidance provided by him during the early stages of the author's graduate studies, and to Dr. W. J. Keeler of Lakehead University for many helpful discussions.

The author also wishes to thank the many other members of the low temperature group, and in particular Messrs. J. E. Ostenson, R. J. Delfs, and J. C. Holste, for their assistance and helpful discussions during the period of this research.

Finally, the author wishes to express his appreciation to his wife, Judy, who, in addition to writing most of the computer programs used in calculating the critical field curves, provided constant encouragement and support. 
133

VII. APPENDIX A: CRITICAL FIELD DATA 
Table 5. Critical field curve data for Th -0.030 at. $\%$ U

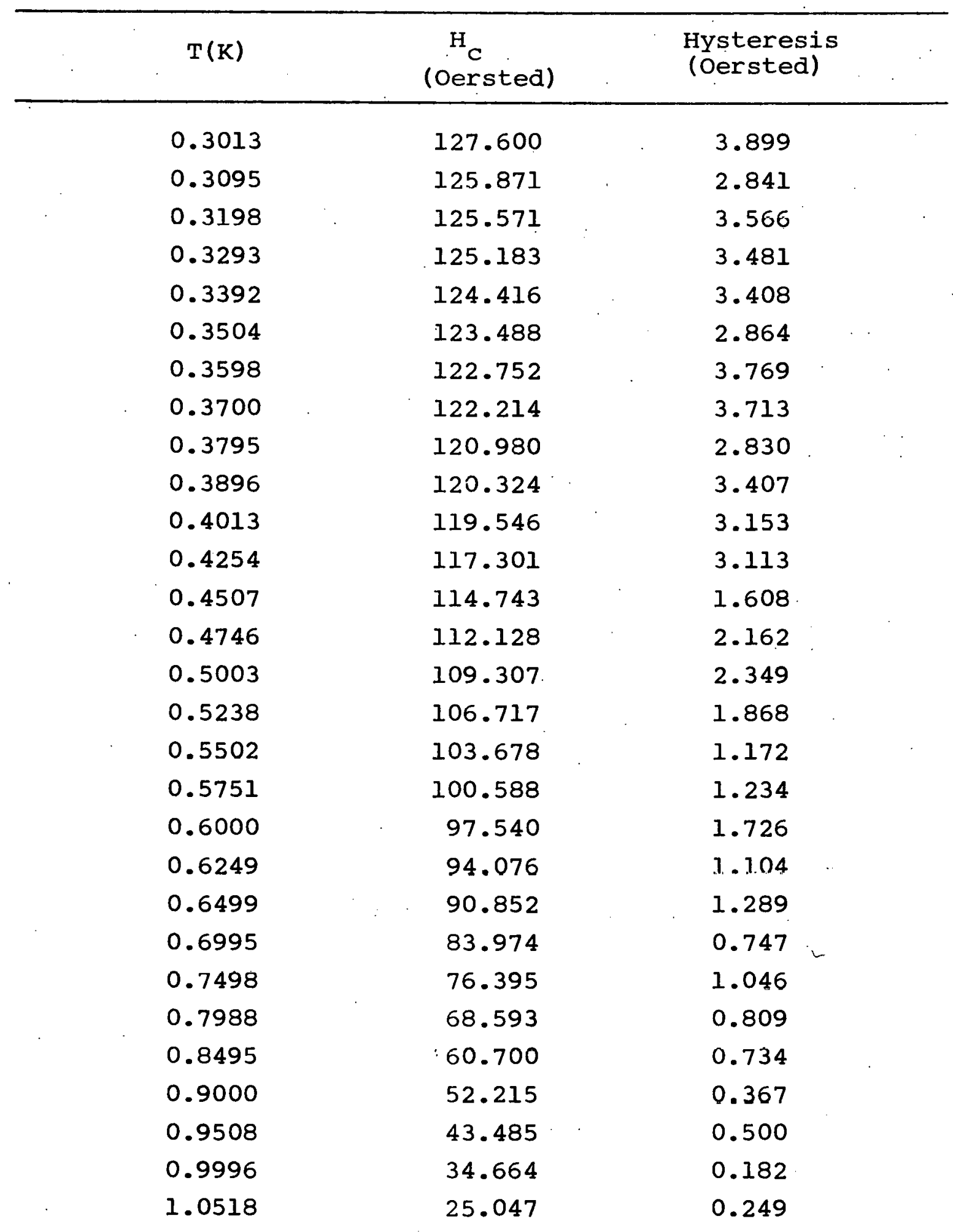


Table 5 (Continued)

\begin{tabular}{lll}
\hline $\mathrm{T}(\mathrm{K})$ & $\begin{array}{c}\mathrm{H}_{\mathrm{C}} \\
\text { (Oersted) }\end{array}$ & $\begin{array}{c}\text { Hysteresis } \\
\text { (Oersted) }\end{array}$ \\
\hline 1.0983 & 16.505 & 0.495 \\
1.1200 & 12.132 & 0.122 \\
1.1292 & 10.387 & 0.116 \\
1.1377 & 8.756 & 0.058 \\
1.1497 & 6.574 & 0.062 \\
\hline
\end{tabular}

Table 6. Critical field curve data for Th - 0.052 at.\% U

\begin{tabular}{ccc}
\hline $\mathrm{T}(\mathrm{K})$ & $\begin{array}{c}\mathrm{H}_{\mathrm{C}} \\
\text { (Oersted) }\end{array}$ & $\begin{array}{c}\text { Hysteresis } \\
\text { (Oersted) }\end{array}$ \\
\hline 0.3013 & 105.897 & 5.139 \\
0.3503 & 101.850 & 4.833 \\
0.4011 & 97.752 & 5.886 \\
0.4507 & 91.925 & 6.118 \\
0.5003 & 86.404 & 4.268 \\
0.5503 & 79.610 & 4.278 \\
0.6001 & 72.862 & 4.039 \\
0.6499 & 65.001 & 2.407 \\
0.6995 & 57.194 & 2.169 \\
0.7497 & 48.692 & 1.479 \\
0.7995 & 40.208 & 0.736 \\
0.8496 & 31.450 & 0.437 \\
0.9000 & 22.128 & 0.493 \\
0.9507 & 12.700 & 0.191 \\
0.9791 & 6.960 & 0.122 \\
\hline
\end{tabular}


Table 7. Critical field curve data for Th - 0.059 at.\% U

\begin{tabular}{|c|c|c|}
\hline$T(K)$ & $\begin{array}{c}\mathrm{H}_{\mathrm{C}} \\
\text { (Oersted) }\end{array}$ & $\begin{array}{l}\text { Hysteresis } \\
\text { (Oersted) }\end{array}$ \\
\hline 0.3028 & 105.775 & 1.183 \\
\hline 0.3095 & 105.192 & 1.240 \\
\hline 0.319 .8 & 104.391 & 1.064 \\
\hline 0.3296 & 103.559 & 1.053 \\
\hline 0.3392 & 102.690 & 0.934 \\
\hline 0.3501 & 101.638 & 0.934 \\
\hline 0.3598 & 100.888 & 0.928 \\
\hline 0.3701 & 99.682 & 0.872 \\
\hline 0.3799 & 98.754 & 0.872 \\
\hline 0.3897 & 97.848 & 1.053 \\
\hline 0.3994 & 96.940 & 0.990 \\
\hline 0.4251 & 94.203 & 0.985 \\
\hline 0.4503 & 90.870 & 0.860 \\
\hline 0.4748 & 88.520 & 0.883 \\
\hline 0.5003 & 85.251 & 0.737 \\
\hline 0.5238 & 82.406 & 0.957 \\
\hline 0.5502 & 78.769 & 0.560 \\
\hline 0.6000 & 72.004 & 0.552 \\
\hline 0.6504 & 64.518 & 0.432 \\
\hline 0.6998 & 56.620 & 0.252 \\
\hline 0.7499 & 48.483 & 0.306 \\
\hline 0.7998 & 39.925 & 0.319 \\
\hline 0.8511 & 31.047 & 0.181 \\
\hline 0.8992 & 22.113 & 0.062 \\
\hline 0.9493 & 12.712 & 0.129 \\
\hline 0.9596 & 10.734 & 0.068 \\
\hline 0.9698 & 8.874 & 0.058 \\
\hline 0.9768 & 7.380 & 0.180 \\
\hline 0.9788 & 7.246 & 0.125 \\
\hline
\end{tabular}


Table 8. Critical field curve data for Th -0.077 at. $\%$ U

\begin{tabular}{lcc}
\hline$T(K)$ & $\begin{array}{c}\mathrm{H}_{C} \\
\text { (Oersted) }\end{array}$ & $\begin{array}{c}\text { HYsteresis } \\
\text { (Oersted) }\end{array}$ \\
\hline 0.3014 & 87.983 & 6.244 \\
0.3503 & 83.257 & 6.679 \\
0.4008 & 77.442 & 5.631 \\
0.4509 & 70.921 & 4.828 \\
0.5002 & 63.954 & 4.138 \\
0.5501 & 56.493 & 3.965 \\
0.6001 & 48.404 & 2.288 \\
0.6500 & 40.171 & 1.800 \\
0.6996 & 31.543 & 0.623 \\
0.7497 & 21.955 & 0.487 \\
0.7997 & 12.635 & 0.321 \\
0.8094 & 10.728 & 0.189 \\
0.8193 & 8.717 & 0.127 \\
0.8298 & 6.759 & 0.185 \\
\hline
\end{tabular}


Table 9. Critical field curve data for Th - 0.102 at.\% U

\begin{tabular}{|c|c|c|}
\hline$T(K)$ & $\begin{array}{c}\dot{\mathrm{H}}_{\mathrm{C}} \\
\text { (Oersted) }\end{array}$ & $\begin{array}{l}\text { Hysteresis } \\
\text { (Oersted) }\end{array}$ \\
\hline 0.3013 & 68.739 & 0.305 \\
\hline 0.3252 & 65.518 & 0.252 \\
\hline 0.3510 & 62.544 & 0.431 \\
\hline 0.3745 & 59.472 & 0.125 \\
\hline 0.4004 & 56.236 & 0.441 \\
\hline 0.4267 & 52.289 & 0.119 \\
\hline 0.4519 & 48.643 & 0.371 \\
\hline 0.4764 & 44.872 & 0.278 \\
\hline 0.5005 & 41.107 & 0.123 \\
\hline 0.5251 & 37.017 & 0.247 \\
\hline 0.5500 & 32.929 & 0.128 \\
\hline 0.5751 & 28.505 & 0.178 \\
\hline 0.6009 & 23.955 & 0.121 \\
\hline 0.6247 & 19.646 & 0.068 \\
\hline 0.6506 & 14.752 & 0.154 \\
\hline 0.6694 & 11.254 & 0.122 \\
\hline 0.6885 & 7.237 & 0.0 \\
\hline
\end{tabular}


Table 10. Critical field curve data for Th -0.127 at.\% U

\begin{tabular}{lcc}
\hline$T(K)$ & $\begin{array}{c}\mathrm{H}_{\mathrm{C}} \\
\text { (Oersted) }\end{array}$ & $\begin{array}{c}\text { Hysteresis } \\
\text { (Oersted) }\end{array}$ \\
\hline 0.3011 & 58.804 & 0.367 \\
0.3252 & 55.918 & 0.565 \\
0.3511 & 52.556 & 0.863 \\
0.3745 & 49.257 & 0.378 \\
0.4003 & 45.470 & 0.306 \\
0.4253 & 41.778 & 0.387 \\
0.4524 & 37.348 & 0.170 \\
0.4762 & 33.409 & 0.242 \\
0.5007 & 29.465 & 0.252 \\
0.5251 & 25.311 & 0.127 \\
0.5500 & 20.749 & 0.255 \\
0.5752 & 16.254 & 0.185 \\
0.6006 & 11.414 & 0.186 \\
0.6204 & 7.524 & 0.059 \\
\hline
\end{tabular}

Table 11. Critical field curve data for Th -0.150 at. $\%$ U

\begin{tabular}{ccc}
\hline$T(K)$ & $\begin{array}{c}\mathrm{H}_{C} \\
\text { (oersted) }\end{array}$ & $\begin{array}{c}\text { Hysteresis } \\
\text { (Oersted) }\end{array}$ \\
\hline 0.3016 & 35.094 & 0.259 \\
0.3251 & 31.066 & 0.241 \\
0.3508 & 26.954 & 0.184 \\
0.3746 & 22.983 & 0.180 \\
0.4003 & 18.634 & 0.133 \\
0.4262 & 13.821 & 0.061 \\
0.4503 & 8.875 & 0.057 \\
0.4599 & 7.109 & 0.127 \\
\hline
\end{tabular}


140

VIII. APPENDIX $B: \mathrm{H}_{0}$ AND $\mathrm{D}(t)$ FROM BCS THEORY 
Table 12. Calculation of $\mathrm{H}_{0}$ for $\mathrm{Th}-0.030$ at.\% $\mathrm{U}$

\begin{tabular}{cccc}
\hline $\begin{array}{c}\mathrm{H}_{\mathrm{C}} \\
\text { (Oersted) }\end{array}$ & $\mathrm{t}^{2}$ & $\mathrm{~h}(\mathrm{BCS})$ & $\begin{array}{c}\mathrm{H}_{0} \\
\text { (Oersted) }\end{array}$ \\
\hline $127.600 \pm 1.950$ & 0.0650 & 0.92905 & $137.345 \pm 2.099$ \\
$125.871 \pm 1.421$ & 0.0686 & 0.92502 & $136.074 \pm 1.536$ \\
$125.571 \pm 1.783$ & 0.0732 & 0.91987 & $136.510 \pm 1.938$ \\
$125.183 \pm 1.741$ & 0.0777 & 0.91482 & $136.839 \pm 1.903$ \\
$124.416 \pm 1.704$ & 0.0824 & 0.90954 & $136.790 \pm 1.873$ \\
$123.488 \pm 1.432$ & 0.0879 & 0.90336 & $136.699 \pm 1.585$ \\
$122.752 \pm 1.885$ & 0.0927 & 0.89796 & $136.701 \pm 2.066$ \\
$122.214 \pm 1.857$ & 0.0980 & 0.89199 & $137.013 \pm 2.082$ \\
$120.980 \pm 1.415$ & 0.1031 & 0.88625 & $136.508 \pm 1.597$ \\
$120.324 \pm 1.704$ & 0.1087 & 0.87993 & $136.743 \pm 1.937$ \\
$119.546 \pm 1.577$ & 0.1153 & 0.87251 & $137.014 \pm 1.807$ \\
$117.301 \pm 1.557$ & 0.1296 & 0.85641 & $136.968 \pm 1.818$ \\
$114.743 \pm 0.804$ & 0.1455 & 0.83857 & $136.832 \pm 0.959$ \\
$112.128 \pm 1.081$ & 0.1613 & 0.82088 & $136.595 \pm 1.317$ \\
$109.307 \pm 1.175$ & 0.1792 & 0.80092 & $136.477 \pm 1.467$ \\
$106.717 \pm 0.934$ & 0.1965 & 0.78175 & $136.510 \pm 1.195$ \\
$103.678 \pm 0.586$ & 0.2168 & 0.75937 & $136.532 \pm 0.772$ \\
$100.588 \pm 0.617$ & 0.2368 & 0.73746 & $136.398 \pm 0.837$ \\
$97.540 \pm 0.863$ & 0.2578 & 0.71462 & $136.492 \pm 1.208$ \\
$94.076 \pm 0.552$ & 0.2796 & 0.69108 & $136.129 \pm 0.799$ \\
$90.852 \pm 0.645$ & 0.3025 & 0.66658 & $136.296 \pm 0.968$ \\
$83.974 \pm 0.374$ & 0.3504 & 0.61594 & $136.335 \pm 0.607$ \\
$76.395 \pm 0.523$ & 0.4026 & 0.56176 & $135.992 \pm 0.931$ \\
& & &
\end{tabular}


Table 13. Calculation of $\mathrm{H}_{0}$ for $\mathrm{Th}-0.052$ at. $\% \mathrm{U}$

\begin{tabular}{cccc}
\hline $\begin{array}{c}\mathrm{H}_{\mathrm{C}} \\
\text { (Oersted) }\end{array}$ & $\mathrm{t}^{2}$ & $\mathrm{~h}(\mathrm{BCS})$ & $\begin{array}{c}\mathrm{H}_{0} \\
\text { (Oersted) }\end{array}$ \\
\hline $105.897 \pm 2.570$ & 0.0885 & 0.90268 & $117.314 \pm 2.847$ \\
$101.850 \pm 2.417$ & 0.1196 & 0.86766 & $117.385 \pm 2.786$ \\
$97.752 \pm 2.943$ & 0.1568 & 0.82592 & $118.355 \pm 3.563$ \\
$91.925 \pm 3.059$ & 0.1980 & 0.78008 & $117.840 \pm 3.921$ \\
$86.404 \pm 2.134$ & 0.2439 & 0.72972 & $118.407 \pm 2.924$ \\
$79.610 \pm 2.139$ & 0.2951 & 0.67448 & $118.032 \pm 3.171$ \\
$72.986 \pm 2.020$ & 0.3509 & 0.61542 & $118.394 \pm 3.282$ \\
\hline
\end{tabular}

Table 14. Calculation of $\mathrm{H}_{0}$ for $\mathrm{Th}-0.059$ at. $\% \mathrm{U}$

\begin{tabular}{cccc}
\hline $\begin{array}{c}\mathrm{H}_{\mathrm{C}} \\
\text { (Oersted) }\end{array}$ & $\mathrm{t}^{2}$ & $\mathrm{~h}(\mathrm{BCS})$ & $\begin{array}{c}\mathrm{H}_{0} \\
\text { (Oersted) }\end{array}$ \\
\hline $105.775 \pm 0.592$ & 0.0891 & 0.90200 & $117.267 \pm 0.656$ \\
$105.192 \pm 0.620$ & 0.0931 & 0.89751 & $117.204 \pm 0.691$ \\
$104.391 \pm 0.532$ & 0.0994 & 0.89040 & $117.241 \pm 0.597$ \\
$103.559 \pm 0.527$ & 0.1056 & 0.88343 & $117.224 \pm 0.596$ \\
$102.690 \pm 0.467$ & 0.1119 & 0.87633 & $117.182 \pm 0.533$ \\
$101.638 \pm 0.467$ & 0.1192 & 0.86811 & $117.080 \pm 0.538$ \\
$100.888 \pm 0.464$ & 0.1259 & 0.86058 & $117.233 \pm 0.539$ \\
$99.682 \pm 0.436$ & 0.1332 & 0.85238 & $116.945 \pm 0.512$ \\
$98.754 \pm 0.436$ & 0.1403 & 0.84438 & $116.954 \pm 0.516$ \\
$97.848 \pm 0.527$ & 0.1476 & 0.83621 & $11 \% .014 \pm 0.630$ \\
$96.940 \pm 0.495$ & 0.1551 & 0.82782 & $117.103 \pm 0.598$ \\
$94.203 \pm 0.493$ & 0.1757 & 0.80483 & $117.048 \pm 0.613$ \\
$90.870 \pm 0.430$ & 0.1971 & 0.78108 & $116.339 \pm 0.551$ \\
$88.520 \pm 0.442$ & 0.2192 & 0.75672 & $116.979 \pm 0.584$ \\
$85.251 \pm 0.369$ & 0.2433 & 0.73037 & $116.723 \pm 0.505$ \\
$82.406 \pm 0.479$ & 0.2667 & 0.70500 & $116.888 \pm 0.679$ \\
$78.769 \pm 0.280$ & 0.2943 & 0.67534 & $116.636 \pm 0.415$ \\
$72.004 \pm 0.276$ & 0.3500 & 0.61635 & $116.823 \pm 0.448$ \\
\hline
\end{tabular}




\section{3}

Table 15. Calculation of $\mathrm{H}_{0}$ for. Th -0.077 at.\% $\mathrm{U}$

\begin{tabular}{cccc}
\hline $\begin{array}{c}\mathrm{H}_{\mathrm{C}} \\
\text { (Oersted) }\end{array}$ & $\mathrm{t}^{2}$ & $\mathrm{~h}(\mathrm{BCS})$ & $\begin{array}{c}\mathrm{H}_{0} \backslash \\
\text { (Oersted) }\end{array}$ \\
\hline $87.983 \pm 3.122$ & 0.1221 & 0.86485 & $101.732 \pm 3.610$ \\
$83.257 \pm 3.340$ & 0.1649 & 0.81687 & $101.922 \pm 4.089$ \\
$77.442 \pm 2.816$ & 0.2158 & 0.76047 & $101.834 \pm 3.703$ \\
$70.921 \pm 2.414$ & 0.2732 & 0.69799 & $101.607 \pm 3.459$ \\
$63.954 \pm 2.069$ & 0.3362 & 0.63087 & $101.374 \pm 3.280$ \\
$56.493 \pm 1.983$ & 0.4066 & 0.55765 & $101.305 \pm 3.556$ \\
\hline
\end{tabular}

Table 16. Calculation of $\mathrm{H}_{0}$ for $\mathrm{Th}-0.102$ at.\% $\mathrm{U}$

\begin{tabular}{cccc}
\hline $\begin{array}{c}\mathrm{H}_{\mathrm{C}} \\
\text { (Oersted) }\end{array}$ & $\mathrm{t}^{2}$ & $\mathrm{~h}(\mathrm{BCS})$ & $\begin{array}{c}\mathrm{H}_{0} \\
\text { (Oersted) }\end{array}$ \\
\hline $68.739 \pm 0.153$ & 0.1725 & 0.80839 & $85.032 \pm 0.189$ \\
$65.518 \pm 0.126$ & 0.2026 & 0.77500 & $84.526 \pm 0.163$ \\
$62.544 \pm 0.216$ & 0.2341 & 0.74041 & $84.472 \pm 0.292$ \\
$59.479 \pm 0.063$ & 0.2665 & 0.70521 & $84.342 \pm 0.089$ \\
$56.236 \pm 0.221$ & 0.3047 & 0.66424 & $84.662 \pm 0.333$ \\
$52.289 \pm 0.060$ & 0.3460 & 0.62057 & $84.260 \pm 0.097$ \\
$48.643 \pm 0.186$ & 0.3880 & 0.57682 & $84.330 \pm 0.322$ \\
\hline
\end{tabular}


Table 17. Calculation of $\mathrm{H}_{0}$ for $\mathrm{Th}-0.127$ at. $\% \mathrm{U}$

\begin{tabular}{cccc}
\hline $\begin{array}{c}\mathrm{H}_{\mathrm{C}} \\
\text { (Oersted) }\end{array}$ & $\mathrm{t}^{2}$ & $\mathrm{~h}(\mathrm{BCS})$ & $\begin{array}{c}\mathrm{H}_{0} \\
\text { (Oersted) }\end{array}$ \\
\hline $58.804 \pm 0.184$ & 0.2093 & 0.76760 & $76.608 \pm 0.240$ \\
$55.918 \pm 0.283$ & 0.2441 & 0.72950 & $76.653 \pm 0.388$ \\
$52.556 \pm 0.432$ & 0.2845 & 0.68584 & $76.630 \pm 0.630$ \\
$49.257 \pm 0.189$ & 0.3237 & 0.64406 & $76.479 \pm 0.293$ \\
$45.470 \pm 0.303$ & 0.3699 & 0.59556 & $76.348 \pm 0.509$ \\
\hline
\end{tabular}


Table 18. $D(t)$ for Th -0.030 at. $\% U$

\begin{tabular}{|c|c|c|}
\hline$t^{2}$ & $\mathrm{~h}$ & $D(t)$ \\
\hline 0.0650 & 0.9341 & -0.0009 \\
\hline 0.0686 & 0.9215 & -0.0099 \\
\hline 0.0732 & 0.9193 & -0.0075 \\
\hline 0.0777 & 0.9164 & -0.0059 \\
\hline 0.0824 & 0.9108 & -0.0068 \\
\hline 0.0879 & 0.9040 & -0.0081 \\
\hline 0.0927 & 0.8986 & -0.0087 \\
\hline 0.0980 & 0.8947 & -0.0073 \\
\hline 0.1031 & 0.8857 & -0.0112 \\
\hline 0.1087 & 0.8808 & -0.0105 \\
\hline 0.1153 & 0.8752 & -0.0095 \\
\hline 0.1296 & 0.8587 & -0.0117 \\
\hline 0.1455 & 0.8400 & -0.0145 \\
\hline 0.1613 & 0.8208 & -0.0179 \\
\hline 0.1792 & 0.8002 & -0.0206 \\
\hline 0.1965 & 0.8035 & -0.0223 \\
\hline 0.2168 & 0.7590 & -0.0242 \\
\hline 0.2368 & 0.7364 & -0.0268 \\
\hline 0.2578 & 0.7141 & -0.0281 \\
\hline 0.2796 & 0.6887 & -0.0317 \\
\hline 0.3025 & 0.6651 & -0.0324 \\
\hline 0.3504 & 0.6147 & -0.0349 \\
\hline 0.4026 & 0.5593 & -0.0381 \\
\hline 0.4581 & 0.5021 & -0.0398 \\
\hline 0.5168 & 0.4444 & -0.0388 \\
\hline 0.5801 & 0.3822 & -0.0377 \\
\hline 0.6474 & 0.3183 & -0.0343 \\
\hline 0.7155 & 0.2538 & -0.0307 \\
\hline 0.7922 & 0.1834 & -0.0244 \\
\hline
\end{tabular}


Table 18 (Continued)

\begin{tabular}{ccc}
\hline$t^{2}$ & $h$ & $D(t)$ \\
\hline 0.8638 & 0.1208 & -0.0154 \\
0.8983 & 0.0888 & -0.01 .29 \\
0.9131 & 0.0760 & -0.0109 \\
0.9269 & 0.0641 & -0.0090 \\
0.9466 & 0.0481 & -0.0053 \\
\hline
\end{tabular}

Table 19. $D(t)$ for Th -0.052 at.\% $U$

\begin{tabular}{lll}
\hline$t^{2}$ & $h$ & $D(t)$ \\
\hline 0.0885 & 0.8977 & -0.0138 \\
0.1196 & 0.8634 & -0.0170 \\
0.1568 & 0.8287 & -0.0145 \\
0.1980 & 0.7793 & -0.0227 \\
0.2439 & 0.7325 & -0.0236 \\
0.2951 & 0.6749 & -0.0300 \\
0.3509 & 0.6177 & -0.0314 \\
0.4116 & 0.5510 & -0.0374 \\
0.4768 & 0.4849 & -0.0383 \\
0.5477 & 0.4128 & -0.0395 \\
0.6229 & 0.3409 & -0.0362 \\
0.7034 & 0.2666 & -0.0300 \\
0.7893 & 0.1876 & -0.0231 \\
0.8808 & 0.1077 & -0.0115 \\
0.9342 & 0.0590 & -0.0068 \\
\hline
\end{tabular}


Table 20. $D(t)$ for $T h-0.059$ at. $\% U$

\begin{tabular}{|c|c|c|}
\hline$\overline{t^{2}}$ & $\mathrm{~h}$ & $D(t)$ \\
\hline 0.0891 & 0.9041 & -0.0068 \\
\hline 0.0931 & 0.8991 & -0.0078 \\
\hline 0.0994 & 0.8923 & -0.0083 \\
\hline 0.1056 & 0.8852 & -0.0092 \\
\hline 0.1119 & 0.8777 & -0.0104 \\
\hline 0.1192 & 0.8687 & -0.0121 \\
\hline 0.1259 & 0.8623 & -0.0118 \\
\hline 0.1332 & 0.8520 & -0.0148 \\
\hline 0.1403 & 0.8441 & -0.0156 \\
\hline 0.1476 & 0.8364 & -0.0160 \\
\hline 0.1551 & 0.8286 & -0.0163 \\
\hline 0.1757 & 0.8052 & -0.0191 \\
\hline 0.1971 & 0.7767 & -0.0262 \\
\hline 0.2192 & 0.7566 & -0.0242 \\
\hline 0.2433 & 0.7287 & -0.0280 \\
\hline 0.2667 & 0.7044 & -0.0289 \\
\hline 0.2943 & 0.6732 & -0.0325 \\
\hline 0.3500 & 0.6155 & -0.0345 \\
\hline 0.4113 & 0.5515 & -0.0372 \\
\hline 0.4761 & 0.4840 & -0.0399 \\
\hline 0.5467 & 0.4144 & -0.0389 \\
\hline 0.6219 & 0.3413 & -0.0368 \\
\hline 0.7042 & 0.2654 & -0.0304 \\
\hline 0.7861 & 0.1890 & -0.0249 \\
\hline 0.8761 & 0.1087 & -0.0152 \\
\hline 0.8952 & 0.0917 & -0.0131 \\
\hline 0.9144 & 0.0759 & -0.0097 \\
\hline 0.9276 & 0.0631 & -0.0093 \\
\hline 0.9314 & 0.0619 & -0.0067 \\
\hline
\end{tabular}


Table 21. $D(t)$ for $T h-0.077$ at. $\% U$

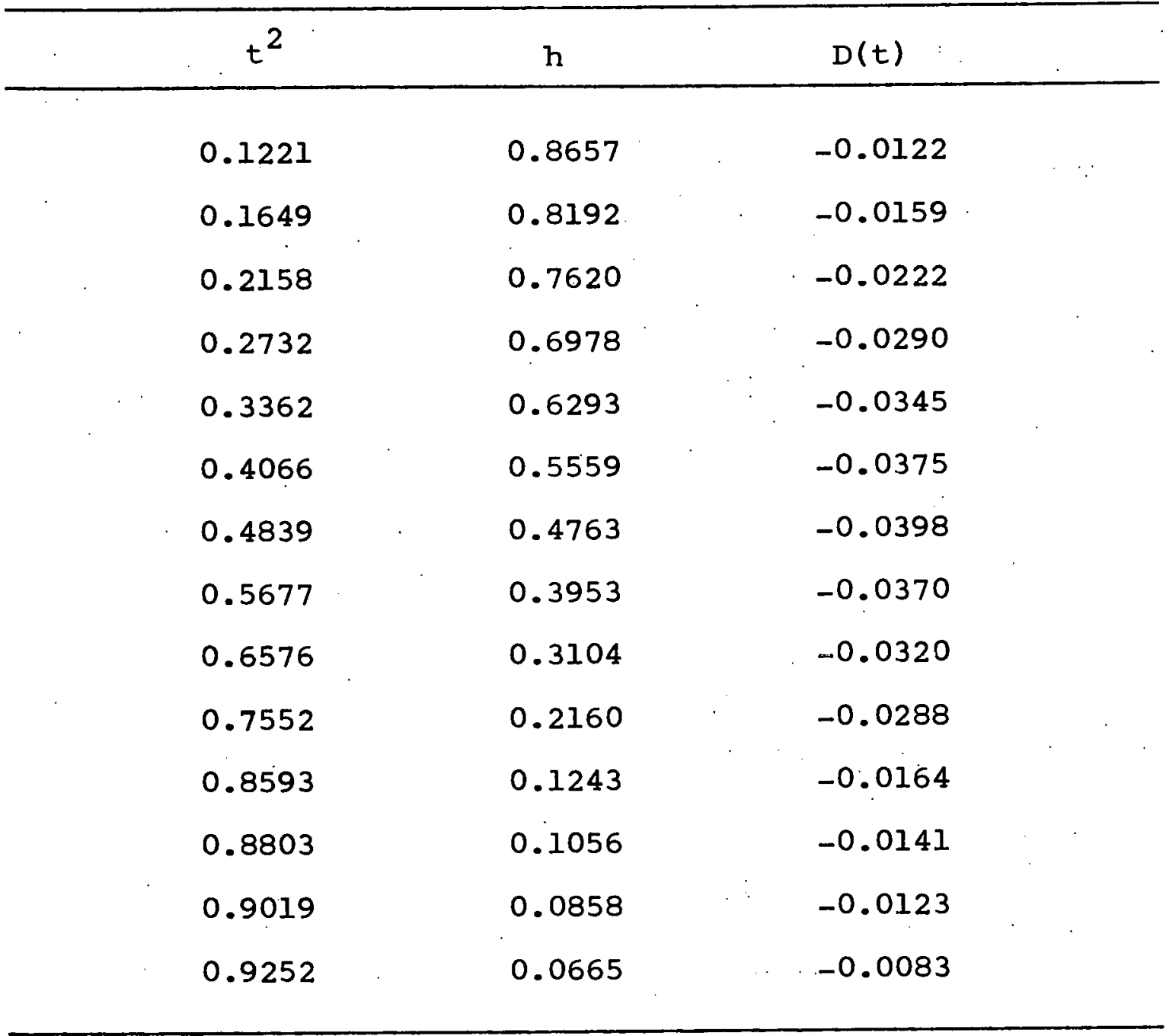


Table 22. $D(t)$ for Th -0.102 at.\% $U$

\begin{tabular}{lll}
\hline$t^{2}$ & $h$ & $D(t)$ \\
\hline 0.1725 & 0.8133 & -0.0142 \\
0.2026 & 0.7752 & -0.0222 \\
0.2341 & 0.7400 & -0.0259 \\
0.2665 & 0.7037 & -0.0298 \\
0.3047 & 0.6654 & -0.0299 \\
0.3460 & 0.6187 & -0.0353 \\
0.3880 & 0.5755 & -0.0365 \\
0.4313 & 0.5309 & -0.0378 \\
0.4761 & 0.4864 & -0.0375 \\
0.5240 & 0.4380 & -0.0380 \\
0.5749 & 0.3896 & -0.0355 \\
0.6285 & 0.3373 & -0.0345 \\
0.6862 & 0.2834 & -0.0304 \\
0.7416 & 0.2324 & -0.0260 \\
0.8044 & 0.1745 & -0.0211 \\
0.8516 & 0.1332 & -0.0152 \\
0.9009 & 0.0856 & -0.0135 \\
\hline & & \\
\hline & & \\
\hline
\end{tabular}


Table 23. $D(t)$ for $T h-0.127$ at.\% $U$

\begin{tabular}{ccc}
\hline$t^{2}$ & $\mathrm{~h}$ & $\mathrm{D}(\mathrm{t})$ \\
\hline 0.2093 & 0.7682 & -0.0225 \\
0.2441 & 0.7305 & -0.0254 \\
0.2845 & 0.6866 & -0.0289 \\
0.3237 & 0.6435 & -0.0328 \\
0.3699 & 0.5940 & -0.0361 \\
0.4175 & 0.5458 & -0.0367 \\
0.4724 & 0.4879 & -0.0397 \\
0.5234 & 0.4365 & -0.0401 \\
0.5787 & 0.3849 & -0.0364 \\
0.6365 & 0.3307 & -0.0328 \\
0.6982 & 0.2711 & -0.0307 \\
0.7637 & 0.2123 & -0.0240 \\
0.8326 & 0.1491 & -0.0183 \\
0.8884 & 0.0983 & -0.0133 \\
\hline
\end{tabular}


151

IX. APPENDIX $c: \mathrm{H}_{0}$ AND $\mathrm{D}(t)$ FROM THE ANISOTROPY CORRECTED BCS THEORY OF CLEM 
Table 24. Calculation of anisotropy corrected $\mathrm{H}_{0}$ for Th - 0.030 at. $\% U$

\begin{tabular}{cccc}
\hline $\begin{array}{c}\mathrm{H}_{\mathrm{C}} \\
\text { (Oersted) }\end{array}$ & $\mathrm{t}^{2}$ & $\mathrm{~h}(\mathrm{BCS}-\mathrm{Clem})$ & $\begin{array}{c}\mathrm{H}_{0} \\
\text { (Oersted) }\end{array}$ \\
\hline $127.600 \pm 1.950$ & 0.0650 & 0.9275 & $137.574 \pm 2.102$ \\
$125.871 \pm 1.421$ & 0.0686 & 0.9234 & $136.313 \pm 1.539$ \\
$125.571 \pm 1.783$ & 0.0732 & 0.9181 & $136.773 \pm 1.942$ \\
$125.183 \pm 1.741$ & 0.0777 & 0.9129 & $137.127 \pm 1.907$ \\
$124.416 \pm 1.704$ & 0.0824 & 0.9075 & $137.098 \pm 1.878$ \\
$123.488 \pm 1.432$ & 0.0879 & 0.9013 & $137.011 \pm 1.589$ \\
$122.752 \pm 1.885$ & 0.0927 & 0.8958 & $137.031 \pm 2.104$ \\
$122.214 \pm 1.857$ & 0.0980 & 0.8898 & $137.350 \pm 2.087$ \\
$120.980 \pm 1.415$ & 0.1031 & 0.8839 & $136.871 \pm 1.601$ \\
$120.324 \pm 1.7 .04$ & 0.1087 & 0.8775 & $137.121 \pm 1.942$ \\
$119.546 \pm 1.577$ & 0.1153 & 0.8699 & $137.425 \pm 1.813$ \\
$117.301 \pm 1.557$ & 0.1296 & 0.8536 & $137.419 \pm 1.824$ \\
$114.743 \pm 0.804$ & 0.1455 & 0.8355 & $137.335 \pm 0.962$ \\
$112.128 \pm 1.081$ & 0.1613 & 0.8174 & $137.176 \pm 1.322$ \\
$109.307 \pm 1.175$ & 0.1792 & 0.7973 & $137.096 \pm 1.474$ \\
$106.717 \pm 0.934$ & 0.1965 & 0.7779 & $137.186 \pm 1.201$ \\
$103.678 \pm 0.586$ & 0.2168 & 0.7553 & $137.267 \pm 0.776$ \\
$100.588 \pm 0.617$ & 0.2368 & 0.7332 & $137.190 \pm 0.842$ \\
$97.540 \pm 0.863$ & 0.2578 & 0.7101 & $137.361 \pm 1.215$ \\
$94.076 \pm 0.552$ & 0.2796 & 0.6864 & $137.057 \pm 0.804$ \\
$90.852 \pm 0.645$ & 0.3025 & 0.6618 & $137.280 \pm 0.975$ \\
$83.974 \pm 0.374$ & 0.3504 & 0.6111 & $137.414 \pm 0.612$ \\
$76.395 \pm 0.523$ & 0.4026 & 0.5568 & $137.204 \pm 0.939$ \\
& & & \\
\hline
\end{tabular}


Table 25. Calculation of anisotropy corrected $\mathrm{H}_{0}$ for Th - 0.059 at.\% U

\begin{tabular}{|c|c|c|c|}
\hline $\begin{array}{c}\mathrm{H}_{\mathrm{C}} \\
\text { (Oersted) }\end{array}$ & $t^{2}$ & $h(B C S-C l e m)$ & $\begin{array}{c}\mathrm{H}_{\mathrm{O}} \\
\text { (Oersted) }\end{array}$ \\
\hline $105.775 \pm 0.592$ & 0.0891 & 0.9003 & $117.489 \pm 0.658$ \\
\hline $105.192 \pm 0.620$ & 0.0931 & 0.8958 & $117.428 \pm 0.692$ \\
\hline $104.391 \pm 0.532$ & 0.0994 & 0.8885 & $117.491 \pm 0.599$ \\
\hline $103.559 \pm 0.527$ & 0.1056 & 0.8815 & $117.480 \pm 0.598$ \\
\hline $102.690 \pm 0.467$ & 0.1119 & 0.8743 & $117.454 \pm 0.534$ \\
\hline $101.638 \pm 0.467$ & 0.1192 & 0.8660 & $117.365 \pm 0.539$ \\
\hline $100.888 \pm 0.464$ & 0.1259 & 0.8584 & $117.530 \pm 0.541$ \\
\hline $99.682 \pm 0.436$ & 0.1332 & 0.8501 & $117.259 \pm 0.513$ \\
\hline $98.754 \pm 0.436$ & 0.1403 & 0.8420 & $117.285 \pm 0.518$ \\
\hline $97.848 \pm 0.527$ & 0.1476 & 0.8337 & $117.366 \pm 0.632$ \\
\hline $96.940 \pm 0.495$ & 0.1551 & 0.8251 & $117.489 \pm 0.600$ \\
\hline $94.203 \pm 0.493$ & 0.1757 & 0.8020 & $117.460 \pm 0.615$ \\
\hline $90.870 \pm 0.430$ & 0.1971 & 0.7780 & $116.799 \pm 0.553$ \\
\hline $88.520 \pm 0.442$ & 0.2192 & 0.7535 & $117.478 \pm 0.587$ \\
\hline $85.251 \pm 0.369$ & 0.2433 & 0.7270 & $117.264 \pm 0.508$ \\
\hline $82.406 \pm 0.479$ & 0.2667 & 0.7013 & $117.505 \pm 0.683$ \\
\hline $78.769 \pm 0.280$ & 0.2943 & 0.6714 & $117.321 \pm 0.417$ \\
\hline $72.004 \pm 0.276$ & 0.3500 & 0.6122 & $117.615 \pm 0.451$ \\
\hline
\end{tabular}


Table 26. Calculation of anisotropy corrected $\mathrm{H}_{0}$ for Th - 0.102 at. $\% U$

\begin{tabular}{cccc}
\hline $\begin{array}{c}\mathrm{H}_{\mathrm{C}} \\
\text { (Oersted) }\end{array}$ & $\mathrm{t}^{2}$ & $\mathrm{~h}(\mathrm{BCS}-\mathrm{Clem})$ & $\begin{array}{c}\mathrm{H}_{\mathrm{O}} \\
\text { (Oersted) }\end{array}$ \\
\hline $68.739 \pm 0.153$ & 0.1725 & 0.8055 & $85.337 \pm 0.190$ \\
$65.518 \pm 0.126$ & 0.2026 & 0.7717 & $84.888 \pm 0.163$ \\
$62.544 \pm 0.216$ & 0.2341 & 0.7369 & $84.874 \pm 0.293$ \\
$59.479 \pm 0.063$ & 0.2665 & 0.7014 & $84.800 \pm 0.090$ \\
$56.236 \pm 0.221$ & 0.3047 & 0.6603 & $85.167 \pm 0.335$ \\
$52.289 \pm 0.060$ & 0.3460 & 0.6165 & $84.816 \pm 0.097$ \\
$48.643 \pm 0.186$ & 0.3880 & 0.5727 & $84.936 \pm 0.325$ \\
\hline
\end{tabular}

Table 27. Calculation of anisotropy corrected $\mathrm{H}_{0}$ for Th - 0.127 at. $\%$ U

\begin{tabular}{cccc}
\hline $\begin{array}{c}\mathrm{H}_{\mathrm{C}} \\
\text { (Oersted) }\end{array}$ & $\mathrm{t}^{2}$ & $\mathrm{H}(\mathrm{BCS}-\mathrm{Clem})$ & $\begin{array}{c}\mathrm{H}_{0} \\
\text { (Oersted) }\end{array}$ \\
\hline $58.804 \pm 0.184$ & 0.2093 & 0.7642 & $76.948 \pm 0.241$ \\
$55.918 \pm 0.283$ & 0.2441 & 0.7259 & $77.033 \pm 0.390$ \\
$52.556 \pm 0.432$ & 0.2845 & 0.6820 & $77.062 \pm 0.633$ \\
$49.257 \pm 0.189$ & 0.3237 & 0.6401 & $76.952 \pm 0.295$ \\
$45.470 \pm 0.303$ & 0.3699 & 0.5915 & $76.872 \pm 0.512$ \\
\hline
\end{tabular}


Table 28. Anisotropy corrected $D(t)$ for Th -0.030 at.\% $U$

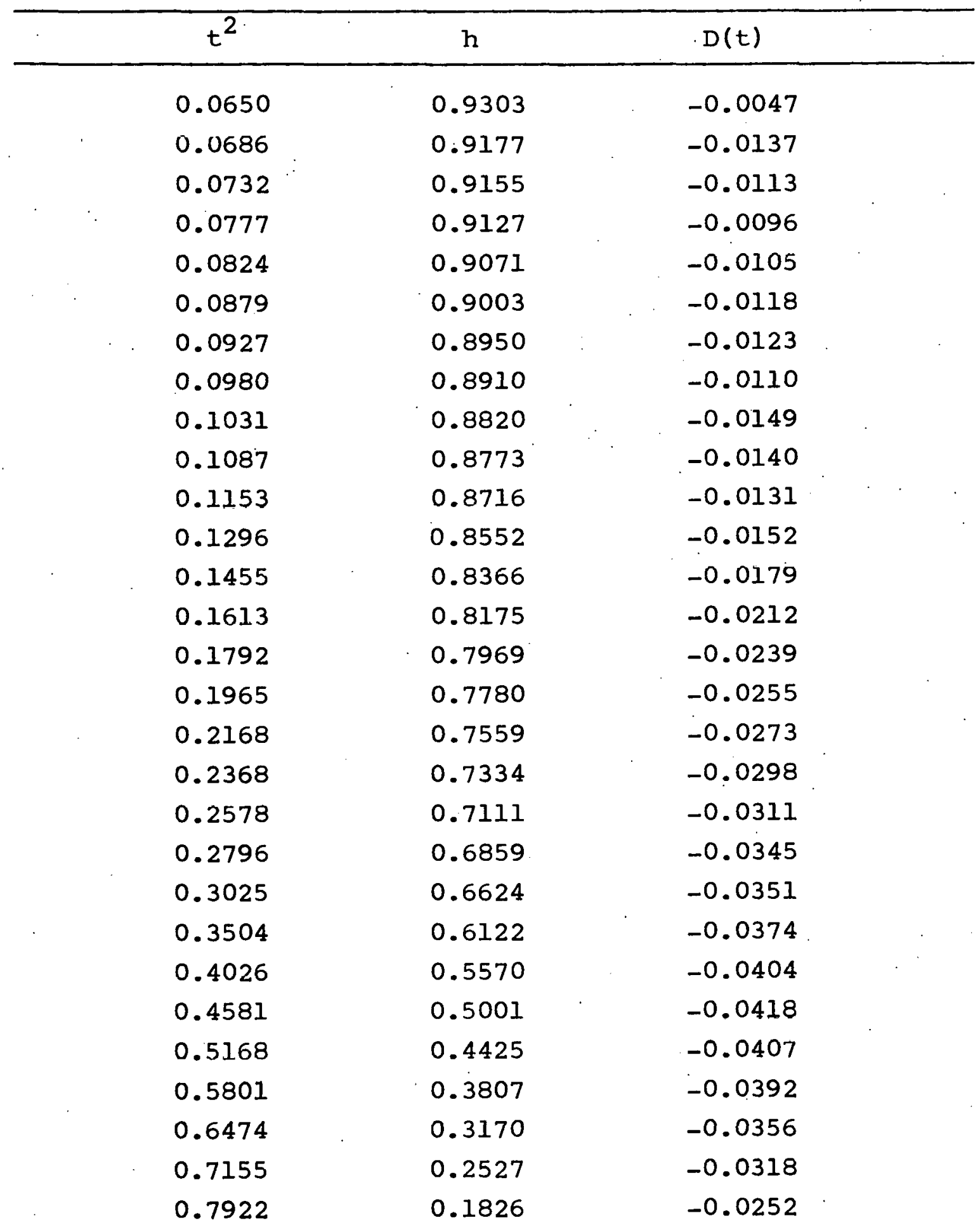


Table 28 (Continued)

\begin{tabular}{ccc}
\hline$t^{2}$ & $h$ & $D(t)$ \\
\hline 0.8638 & 0.1203 & -0.0159 \\
0.8983 & 0.0885 & -0.0132 \\
0.9131 & 0.0757 & -0.0112 \\
0.9269 & 0.0638 & -0.0093 \\
0.9466 & 0.0479 & -0.0055 \\
\hline
\end{tabular}

Table 29. Anisotropy corrected $D(t)$ for Th -0.059 at. $\% U$

\begin{tabular}{lll}
\hline$t^{2}$ & $h$ & $D(t)$ \\
\hline 0.0891 & 0.9010 & -0.0099 \\
0.0931 & 0.8961 & -0.0108 \\
0.0994 & 0.8892 & -0.0114 \\
0.1056 & 0.8822 & -0.0122 \\
0.1119 & 0.8748 & -0.0133 \\
0.1192 & 0.8658 & -0.0150 \\
0.1259 & 0.8595 & -0.0146 \\
0.1332 & 0.8491 & -0.0177 \\
0.1403 & 0.8412 & -0.0185 \\
0.1476 & 0.8335 & -0.0189 \\
0.1551 & 0.8258 & -0.0191 \\
0.1757 & 0.8025 & -0.0218 \\
0.1971 & 0.7741 & -0.0288 \\
0.2192 & 0.7540 & -0.0268 \\
0.2433 & 0.7262 & -0.0305 \\
0.2667 & 0.7020 & -0.0313 \\
0.2943 & 0.6710 & -0.0347 \\
0.3500 & 0.6134 & -0.0366 \\
0.4113 & 0.5496 & -0.0391
\end{tabular}


Table 29 (Continued)

\begin{tabular}{ccc}
\hline$t^{2}$ & $h$ & $D(t)$ \\
\hline 0.4761 & 0.4823 & -0.0416 \\
0.5467 & 0.4130 & -0.0403 \\
0.6219 & 0.3401 & -0.0380 \\
0.7042 & 0.2645 & -0.0313 \\
0.7861 & 0.1884 & -0.0255 \\
0.8761 & 0.1083 & -0.0156 \\
0.8952 & 0.0914 & -0.0134 \\
0.9144 & 0.0756 & -0.0100 \\
0.9276 & 0.0629 & -0.0095 \\
0.9314 & 0.0617 & -0.0069 \\
\hline
\end{tabular}

Table 30. Anisotropy corrected $D(t)$ for Th -0.102 at.\% U

\begin{tabular}{ccc}
\hline$t^{2}$ & $h$ & $D(t)$ \\
\hline 0.1725 & 0.8055 & -0.0220 \\
0.2026 & 0.7717 & -0.0257 \\
0.2341 & 0.7369 & -0.0290 \\
0.2665 & 0.7014 & -0.0321 \\
0.3047 & 0.6603 & -0.0350 \\
0.3460 & 0.6165 & -0.0375 \\
0.3880 & 0.5724 & -0.0396 \\
0.4313 & 0.5281 & -0.0406 \\
0.4761 & 0.4838 & -0.0401 \\
0.5240 & 0.4356 & -0.0404 \\
0.5749 & 0.3875 & -0.0376 \\
0.6285 & 0.3355 & -0.0360 \\
0.6862 & 0.2819 & -0.0319
\end{tabular}


Table 30 (Continued)

\begin{tabular}{ccc}
\hline$t^{2}$ & $h$ & $D(t)$ \\
\hline 0.7416 & 0.2312 & -0.0272 \\
0.8044 & 0.1736 & -0.0220 \\
0.8516 & 0.1324 & -0.0160 \\
0.9009 & 0.0852 & -0.0139 \\
\hline
\end{tabular}

Table 31. Anisotropy corrected $D(t)$ for Th - 0.127 at.\% $U$

\begin{tabular}{ccc}
\hline$t^{2}$ & $h$ & $D(t)$ \\
\hline 0.2093 & 0.7640 & -0.0267 \\
0.2441 & 0.7265 & -0.0294 \\
0.2845 & 0.6828 & -0.0327 \\
0.3237 & 0.6399 & -0.0364 \\
0.3699 & 0.5907 & -0.0394 \\
0.4175 & 0.5428 & -0.0397 \\
0.4724 & 0.4852 & -0.0424 \\
0.5234 & 0.4340 & -0.0426 \\
0.5787 & 0.3828 & -0.0385 \\
0.6365 & 0.3288 & -0.0347 \\
0.6982 & 0.2696 & -0.0322 \\
0.7637 & 0.2112 & -0.0251 \\
0.8326 & 0.1483 & -0.0191 \\
0.8884 & 0.0977 & -0.0139 \\
\hline
\end{tabular}

